COST, PRECISION, AND TASK STRUCTURE IN AGGRESSION-BASED ARBITRATION FOR MINIMALIST ROBOT COOPERATION

\author{
A Thesis \\ by \\ TANUSHREE MITRA
}

Submitted to the Office of Graduate Studies of Texas A\&M University in partial fulfillment of the requirements for the degree of

MASTER OF SCIENCE

August 2011

Major Subject: Computer Science 


\title{
COST, PRECISION, AND TASK STRUCTURE IN AGGRESSION-BASED ARBITRATION FOR MINIMALIST ROBOT COOPERATION
}

\author{
A Thesis \\ by \\ TANUSHREE MITRA

\begin{abstract}
Submitted to the Office of Graduate Studies of Texas A\&M University in partial fulfillment of the requirements for the degree of

MASTER OF SCIENCE
\end{abstract}

Approved by:

Chair of Committee, Dylan Shell

Committee Members, Dezhen Song

Gil Rosenthal

Head of Department, Duncan M. Walker

August 2011

Major Subject: Computer Science 


\begin{abstract}
Cost, Precision, and Task Structure in Aggression-based

Arbitration for Minimalist Robot Cooperation. (August 2011)

Tanushree Mitra, B.Tech., Sikkim Manipal Institute of Technology

Chair of Advisory Committee: Dr. Dylan Shell
\end{abstract}

Multi-robot systems have the potential to improve performance through parallelism. Unfortunately, interference often diminishes those returns. Starting from the earliest multi-robot research, a variety of arbitration mechanisms have been proposed to maximize speed-up. Vaughan and his collaborators demonstrated the effectiveness of an arbitration mechanism inspired by biological signaling where the level of aggression displayed by each agent effectively prioritizes the limited resources. But most often these arbitration mechanisms did not do any principled consideration of environmental constraints or task structure, signaling cost and precision of the outcome. These factors have been taken into consideration in this research and a taxonomy of the arbitration mechanisms have been presented. The taxonomy organizes prior techniques and newly introduced novel techniques. The latter include theoretical and practical mechanisms (from minimalist to especially efficient). Practicable mechanisms were evaluated on physical robots for which both data and models are presented. The arbitration mechanisms described span a whole gamut from implicit (in case of robotics, entirely without representation) to deliberatively coordinated (via an established Biological model, reformulated from a Bayesian perspective).

Another significant result of this thesis is a systematic characterization of system performance across parameters that describe the task structure: patterns of interference are related to a set of strings that can be expressed exactly. This analysis of the domain has the important (and rare) property of completeness, i.e., all possible ab- 
stract variations of the task are understood. This research presents efficiency results showing that a characterization for any given instance can be obtained in sub-linear time. It has been shown, by construction, that: (1) Even an ideal arbitration mechanism can perform arbitrarily poorly; (2) Agents may manipulate task-structure for individual and collective good; (3) Task variations affect the influence that initial conditions have on long-term behavior; (4) The most complex interference dynamics possible for the scenario is a limit cycle behavior. 
To mom and dad who have always provided me words of encouragement and support. 


\section{ACKNOWLEDGMENTS}

Special thanks to Dr. Dylan Shell who has been such a great advisor and a source of constant support and knowledge. 


\section{TABLE OF CONTENTS}

CHAPTER

Page

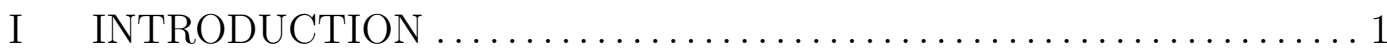

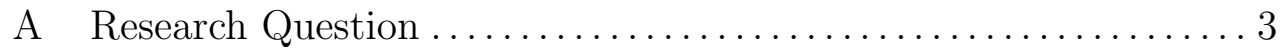

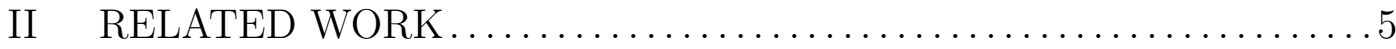

III TAXONOMY OF ARBITRATION METHODS $\ldots \ldots \ldots \ldots \ldots \ldots . \ldots 8$

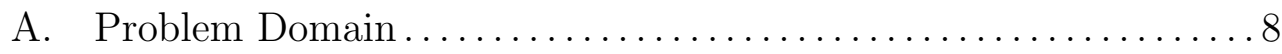

B. Arbitration Methods ............................... 8

C. Taxonomy of Spatial Conflict Resolution Methods.............10

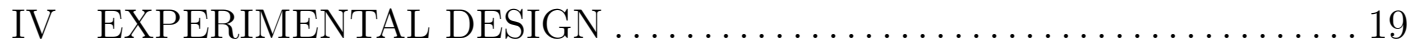

A. Physical Robot Interference $\ldots \ldots \ldots \ldots \ldots \ldots \ldots \ldots \ldots \ldots \ldots$

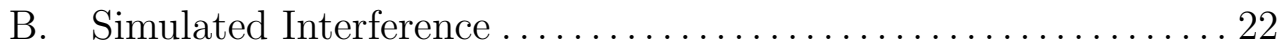

1. Models of motion without interference ..................23

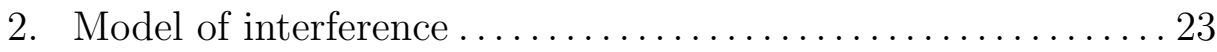

a. Dominance model ............................. 26

b. Aggression model ............................... 28

V COMPARATIVE STUDY OF INTERFERENCE MODELS ..........30

A. Aggressive Interaction and Linear Dominance ................ 30

B. Cutting Your Losses ............................... 34

C. Random Walk ....................................... 35

VI INTERFERENCE — VARYING TASK RATIO AND GIR-

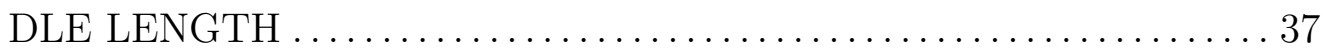

A. Dominance Model...................................37

B. Aggression Model .................................. 41

VII AN ANALYSIS OF SHARED GIRDLE INTERFERENCE

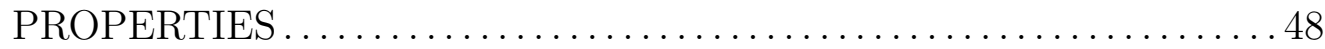


VIII DECISION TREE ENCODING OF ARBITRATION MODELS ......56

A. Definitions ........................................ 56

B. Decision Tree Encodings of Arbitration Models...............60

C. Interference Patterns Generated from Models of Interference....65

D. Relative Cost of an Interference Pattern................... 71

IX BAYESIAN APPROACH TO RESOURCE ARBITRATION ..........74

A. Motivation for Bayesian Approach to Assessment...............74

B. Assessment of Local Investment in Binary Robot Arbitration ...76

$\mathrm{X} \quad$ DISCUSSION AND FUTURE WORK $\ldots \ldots \ldots \ldots \ldots \ldots \ldots \ldots \ldots$

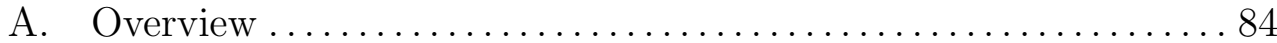

B. Bayesian Inference Model ............................ 85

C. Cooperation versus Coordination....................... 85

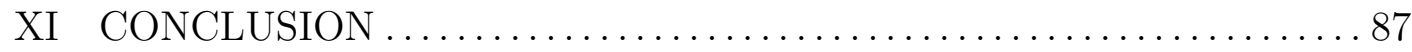

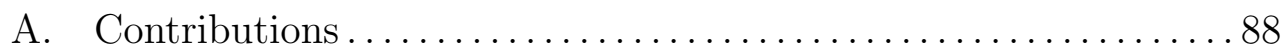

REFERENCES ..........................................91

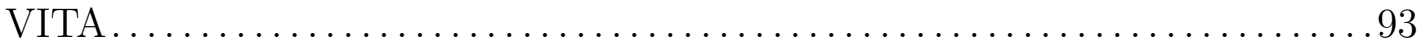




\section{LIST OF TABLES}

TABLE

Page

1 Summary of arbitration methods . . . . . . . . . . . . . 18

2 Strings generated from a dynamic deterministic model . . . . . . . . 60

$3 \quad$ Strings generated from a dynamic probabilistic model . . . . . . . . . 64

4 Strings generated from a static arbitration model . . . . . . . . . . 64

$5 \quad$ String mapping between dynamic deterministic and dynamic probabilistic models . . . . . . . . . . . . . . . . . . . . 65

6 Relative likelihood of occurrence of a string generated from a dynamic probabilistic model . . . . . . . . . . . . . . . 71 
TABLE Page

I Summary of arbitration methods $\ldots \ldots \ldots \ldots \ldots \ldots \ldots \ldots \ldots \ldots \ldots \ldots \ldots \ldots \ldots \ldots$

II Strings generated from a dynamic deterministic model $\ldots \ldots \ldots \ldots \ldots 61$

III Strings generated from a dynamic probabilistic model . . . . . . . . . . 65

IV Strings generated from a static arbitration model $\ldots \ldots \ldots \ldots \ldots \ldots \ldots 6$

V String mapping between dynamic deterministic and dynamic probabilistic

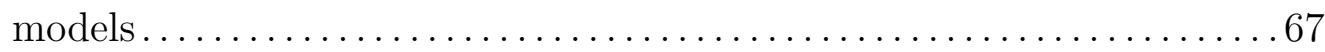

VI Relative likelihood of occurrence of a string generated from a dynamic probabilistic model. .............................. 72 
FIGURES

Page

1 Sketch of the task environment navigated by the robots. The arrows show the direction of motion of the robots $R_{A}$ and $R_{B}$. The flags mark the start and end points for completing one traversal tasks by each robot. The common traversal region of the robots is a narrow corridor, which we term as the "girdle". Spatial interference takes place when both $R_{A}$ and $R_{B}$ attempt to move through the girdle from the opposite directions in the

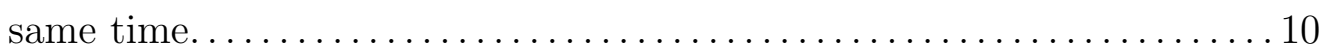

2 Physical robots traversing a narrow corridor at the same time results in

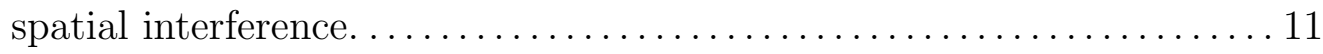

3 Four different arbitration mechanisms...................... 12

4 Robot $R_{B}$ 's wins with respect to the position of encounter is plotted.The data was obtained by running physical robot experiments for different girdle lengths. The vertical axis is the probability that $R_{B}$ wins the encounter. Thus the vertical axis value is 1 when $R_{B}$ wins an encounter and is 0 when it loses. While plotting, all girdle lengths are normalized across a $(0,1]$ scale. We then obtain the best fit of this data as shown

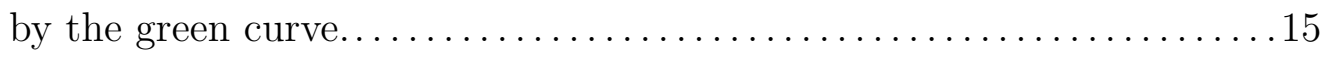

5 The random walk arbitration was done over 1000 different positions inside the girdle of length 5 meters. The fit of the probability of winner with respect to the encounter position was found to be linear. .......... 16

6 Time taken for random walk arbitration with respect to the position of encounter..................................... 17

7 Interference resolution in physical robots by aggressive interactions. . . 20

8 Interference resolution in physical robots by follow of linear dominance

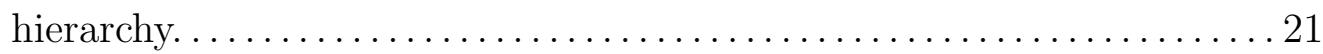


$7 \quad$ Interference resolution in physical robots by aggressive interactions. (a). Two robots approach each other in a narrow girdle, (b). Two robots bump into each other (spatial interference), (c), (d). Each robot backs by a distance inversely proportional to its local investment inside the girdle, (e). The robots move forward after they backing, (f). They bump into each other again, (g), (h). They back proportional to their local investment so far, (i). They move forward and bump into each other again, (j), (k), (l). The losing robot is pushed out of the girdle giving way to the winner.

Interference resolution in physical robots by follow of linear dominance hierarchy. (a). Two robots approach each other in a narrow girdle, (b). Two robots bump into each other (spatial interference), (c), (d), (e). The dominant robot stands ground and the dominated robot turns around to move out of the girdle, (f), (g), (h). The dominated robot leaves the girdle giving right of way to the dominator. . . . . . . . . . . . . . . . .

$9 \quad$ Task Environment navigated by the robots. The initial positions correspond to the positions from where the robots start their traversal for the first time. The flags mark the start and end points for completing one traversal. For the very first traversal, $I_{A}$ and $I_{B}$ are the initial distances from the start point. A single traversal includes traveling in the non-shared region, (marked as $T_{A}$ and $T_{B}$ for robots $R_{A}$ and $R_{B}$ respectively) and moving in the shared common space (termed as the girdle and marked as $G$ ). . . . 22

Task completion times. . . . . . . . . . . . . . .

11 Measured versus true girdle movements. . . . . . . . . . . . . . 24

$12 \quad$ Physical robot data. . . . . . . . . . . . . . . . . 25

13 Simulated using the parameters described above. . . . . . . . . . .

14 Time (length normalized) to resolve an interaction with the dominance model. . . . . . . . . . . . . . . . . . . . 
16 Simulated length normalized time for dominance interactions . . . . .

17 Time (length normalized) to resolve an interaction with the aggression fight model. . . . . . . . . . . . . . .

18 Simulated length normalized time for aggressive fights. . . . . . . . .

19 Average task times of $\mathrm{R}_{A}$ and $\mathrm{R}_{B}$ with varying girdle lengths, fixed task ratio 25:38. . . . . . . . . . . . . . . . . . 31

20 Duration of aggressive interaction with varying task ratio. . . . . . .

21 The intuition behind the "cutting your losses" strategy is illustrated via an example of the aggression-based interaction. The sign of the single-time gain (denoted $\Delta$ in the graphs) indicates a likely win or loss. Waiting longer before measuring $\operatorname{sgn}(\Delta)$ reduces the estimate in the error due to the "escalation" dynamics. . .

22 Random walk - The large number of collisions illustrate the high cost involved. The horizontal-axis shows the difference in the local investment of $\mathrm{R}_{B}$ to that of $\mathrm{R}_{A}$ at the first instance of encounter in the girdle. The winner is determined on the basis of the backing distance drawn from a random distribution throughout the duration of interaction. . . . . . . . . . . . . . . .

23 Dominance for girdle length $=30 \mathrm{~m}, \mathrm{R}_{A}$ is dominator, horizontalaxis shows $R_{A}$ 's task length, vertical-axis that of $\mathrm{R}_{B}$. (a)Color bars shows the relative number of encounters,(b),(c) Color bar shows the relative number of laps finished when at least one of the robots completes 150 laps. Points A to F are detailed in Figure 9.

24 Dominance for girdle length $=30 \mathrm{~m}, \mathrm{R}_{A}$ is dominator. The girdle proportions are with respect to the position of robot $\mathrm{R}_{A}$. TA corresponds to the task length of $\mathrm{R}_{A}$, and $\mathrm{TB}$ to that of $\mathrm{R}_{B} \ldots \ldots$.

25 Collective performance varying girdle length. The horizontal-axis shows $\mathrm{R}_{A}$ 's task length and vertical-axis that of $\mathrm{R}_{B}$. Color bars show the relative time to complete 100 navigation tasks in a row. . . 
26 Collective vs. individual performance for girdle length $=30 \mathrm{~m}$. The horizontal-axis shows $\mathrm{R}_{A}$ 's task length and vertical-axis that of $\mathrm{R}_{B}$. Color bars show the relative measurements when 100 navigation tasks are completed in a row. . . . . . . . . . . . . . .

27 Aggression GL30. The girdle proportion are with respect to the position of robot $\mathrm{R}_{A} \ldots \ldots \ldots \ldots \ldots \ldots$

28 Decision tree encoding when the arbitration is dynamic deterministic or dynamic probabilistic. . . . . . . . . . . . . . .

29 Decision tree encoding when arbitration mechanism is static deterministic. . . . . . . . . . . . . . . . . . .

30 Decision tree encoding when the arbitration mechanism is static probabilistic. . . . . . . . . . . . . . . . . . . 64

31 Cost of an interference when arbitration mechanism is dynamic is proportional to the arbitration time and is at its maximum when the encounter is near the center of the girdle. . . . . . . . . .

$32 \quad$ Probability distribution of measured $\Delta$ for a given true $\Delta$. The distributions over a set of 11 observations are shown. The observed $\Delta$ gets closer to the true $\Delta$ with lesser standard deviation with each successive observation. . . . . . . . . . . . . .

33 Probability distribution of estimated local investment. Here $R_{A}$ estimates its opponent $R_{B}$ 's local investment. The distributions are over a set of 11 observations of $\Delta$ and the probability distribution of the estimate is updated with each successive observations. . .

34 Probability distribution of estimated local investment. Here $R_{B}$ estimates its opponent $R_{A}$ 's local investment. The distributions are over a set of 11 observations of $\Delta$ and the probability distribution of the estimate is updated with each successive observations. . .

35 Error in the estimation decreases with increased observation of $\Delta_{O}$. . 


\section{CHAPTER I}

\section{INTRODUCTION}

Spatial interference is a common and important phenomenon in navigation tasks involving multiple robots. When autonomous robots have an incentive to cooperate, a worthwhile question is how best to mitigate the negative effects of this contention. Motivated by the methods which animals employ to compete for resources, Vaughan and his collaborators ( $c f .[1]$, [2], and [3]) have shown how displays of stylized aggression can effectively resolve resource conflicts in a multi-robot transportation task. That work has produced increasingly effective methods for assessing the level of aggression that an individual agent should exhibit in order to prioritize the limited resource effectively. One way of determining the amount of aggression to be displayed is to make it proportional to the amount of investment an agent does in traversing a shared region. The level of aggression displayed shows how fit an agent is to win the resource. The fitness of an agent may depend on one or several factors. For example, it could be proportional to the amount of investment done in the shared resource, or the priority of the task being performed or may even depend on the past performance of an agent. In our study of robotic spatial interference scenario the fitness is proportional to the amount of investment done in crossing the shared region before a robot encounters an opponent robot from the opposite direction.

However, determining the correct fitness of an individual at a particular time is just one aspect of effective conflict resolution. Results of this thesis demonstrate that additional factors also contribute to conflict resolution and its effectiveness depends on them in a critical way:-

The journal model is IEEE Transactions on Automatic Control. 
- Cost versus precision of the arbitration mechanism - when agents locally estimate their own fitness and try to communicate it during resource arbitration, some communication cost is incurred. The mechanics of this procedure influence the utility of communicating an agent's fitness in the first place. The analogous biological mechanism is directly concerned with the interplay of signal precision and cost: aggressive displays allow animals to assess the strength of their resource competitors before they decide to engage in a costly fight [4]. There is also the question of how long the communication should last in order to make a good enough estimate of the opponent's fitness and how good (or accurate) the estimate is.

- Static versus dynamic conflict resolution - Static arbitration need not have any cost involved in communicating fitness levels, since the abilities of agents are pre-determined and fixed and the level of fitness does not change during the course of navigation. On the other hand, dynamic conflict resolution can have varying levels of fitness and there is an inherent cost for communicating an agent's fitness.

- Properties of the shared resource for which the agents are competing - this can affect, among other things, the cost of communicating its fitness and what constitutes a worthwhile investment.

- The task which each agent is assigned to perform - this can be coupled through the shared resource. This coupling, effects individual and collective dynamics.

- The inherent noise in the communication channel - the role of noise comes into play in dynamic arbitration mechanisms when agents try to communicate their fitness level through a noisy channel. This may cause inaccuracies in estimating 
an opponent's communicated level of fitness. This in turn may lead to extended conflicts till an agent is confident enough in its estimate. However, noise can be beneficial in breaking symmetry, a situation which often occurs when agents have identical fitness levels.

In fact our results show that the properties of the resource influence the cost of arbitration with aggressive displays, so much so that it might not be worth it for an agent to display its fitness at all. Instead it might be worthwhile to follow a static strategy. Varying the properties of assigned task, the frequency of spatial interference and the cost incurred in resolving it varies significantly. Even memory of past interactions with respect to the task structure and properties of the resource can be used to improve future task performance. Moreover, inherent noise in the communication channel can influence the accuracy of fight outcome, in terms of who would be the rightful winner given their true abilities.

Apart from identifying the significant factors influencing robotic spatial interference, this thesis also categorizes different arbitration mechanisms ranging from the most implicit minimalist arbitration where arbitration hardly has any representation to the most informed and coordinated explicit arbitration. Further more, this research also characterizes system performance across parameters that describe the task structure, specifically, patterns of interference which can be expressed exactly as a set of strings. This analysis of the domain has the important (and rare) property of completeness, i.e., all possible abstract variations of the task are understood.

\section{A. Research Questions}

Broadly, the research questions which this thesis addresses are as follows:-

- What are the chances that any interference will occur? 
- Given some spatial interaction, how likely is it that the subsequent interference will result in interference?

- Are there favorable conditions that prevent or ameliorate the cost of future interference?

- What factors should be taken into consideration while choosing an arbitration mechanism for interference resolution?

- Can the choice of arbitration mechanism decide the frequency of interference for subsequent interactions?

- Can we identify interference patterns for any given arbitration mechanism?

- Can we model the arbitration mechanisms both as implicit with very little representation and as explicit with higher information communication and coordination?

In the next section we present the work done by roboticists in the domain of robotic spatial interference and also by biologists in the domain of animal conflicts over shared resources. 


\section{CHAPTER II}

\section{RELATED WORK}

Goldberg and Matarić [5] have suggested using interference as a tool for evaluating multi-robot controllers, viz. identifying trade-offs between performance time and interference. Beyond identifying the general importance of interference in robot systems, their work showed that a dominance hierarchy is one strategy that can reduce interference in some foraging scenarios.

Vaughan et.al [1] inspired by Konrad Lorenz's [6] work on aggressive behaviors in animals used "aggressive display" strategies to resolve a shared resource conflict, without causing any physical damage to the robots. In order to prevent intra-species physical fights over a shared resource, animals have evolved several strategies. One such strategy is to cue the opponent about its strength and make it submit without engaging in a physical combat. Such displays of an animal's aggressiveness can be termed as "aggressive displays" and is the key to Vaughan et.al's method of arbitration. For the robots there are several alternative methods of choosing the level of aggression. They performed simulation experiments of robotic spatial navigation, choosing different methods for setting aggression levels. They compared the performance of these methods by measuring the number of task traversals completed in each case, where a higher number denoted better performance. Their data showed that the performance is same no matter how the aggression level is set, be it fixed linear dominance hierarchy where aggression level is preset beforehand or dynamically set aggression level, based on the amount of free space behind the robot or even when aggression is determined at random. However in all these methods the robots completed more transportation trips than when the system lacked any arbitration. In contrast our data show that one strategy can be preferable to the other, but exactly 
which will depend on the shared resource and also on the individual task dynamics.

Brown [2] introduced the concept of "rational aggression" where the level of aggression is determined by the amount of time spent by the robot in performing its task, also called the investment made by a robot. Under certain conditions, determining the level aggression on the basis of a robot's global task investment provides a significant performance improvement over a randomly selected outcome.

Zuluaga and Vaughan [3] further improved this performance by basing the level of aggression on the investment in the shared resource, also called the local investment. In terms of robots navigating in an environment this would be equivalent to the amount of time spent in crossing the shared space before bumping into an opponent robot.

Additionally, in their work Goldberg and Matarić [5] suggested that changing the environment could play a role in altering interference properties. This manifests itself in [2], where changes in the (simulated) environment produced large standard deviations in their results. Part of our work is an attempt to analyze and mitigate interference under systematic variation of the environment.

On the other hand biologists such as Enquist and Leimar [7], [4] proposed a "Sequential Assessment Model" (SAM) to model the fighting behavior in animals when they engage in extended contests. SAM consists of repeated interactions where in each interaction the animal obtains some information about the true fighting ability of its opponent. At the very start of the contest an animal has an initial estimate of the fighting ability of its opponent and this estimate gets better and closer to the true fighting ability with each successive interaction. Additionally at each interaction step the animal computes its expected utility based on the information acquired so far and thereby takes the decision whether to continue further interactions or retreat from the contest. The decision is made so as to maximize the expected utility. We draw 
inspiration from Enquist and Leimar's work to come up with deliberately coordinated arbitration mechanism where a robot estimates the fitness of its opponent in repeated interactions.

Thus on one hand an aggression method can be as simple and uninformed as in the case of a randomly set aggression level or it can be as highly coordinated and well informed as an assessment based arbitration method. This motivates our study of this entire spectrum of arbitration mechanisms and propose a taxonomy of arbitration methods. The taxonomy is presented in the next section. 


\section{CHAPTER III}

\section{TAXONOMY OF ARBITRATION METHODS}

\section{A. Problem Domain}

The focus of this thesis is binary robot interference and physical interference takes place when multiple robots try to access the same floor space at the same time. Such interferences fall into the category of same place (SP), same time (ST)

[5]. Figure 1 shows the sketch of an environment where such types of interferences occur. The common traversal region is not wide enough for both robots to pass through it from opposite directions at the same time. Figure 2 shows an instance of spatial interference when two robots try to pass through a narrow corridor, resulting in spatial interference.

In the event of spatial interference there should be some way to arbitrate the resource without causing physical damage to the robots. Several methods of resource arbitration have been proposed. The following section discusses some of these earlier methods. We have also devised some new methods which are unique from the earlier methods either in a way of being more sophisticated or more simple and minimalistic.

\section{B. Arbitration Methods}

A resource arbitration method determines how the level of aggression of a robot should be set, which in turn determines how the resource will be arbitrated. The following lists some arbitration methods:-

Vaughan's random aggression: Agents pick a random aggression at each encounter, resulting in a random outcome.

Vaughan's personal space method: The level of aggression is determined by the 
amount of free space visible to the robot behind it in the event of spatial interference.

Rational aggression based on local task investment: Modeled after [3] this is an aggressive interaction based on local task investment, where the robot "displays its aggression" by moving backward a distance inversely proportional to the distance traveled so far in the constrained region, and then moving forward until it bumps again. The robot behavior follows the steps shown in Figure 3(a).

Linear dominance hierarchy: A fixed aggression level is assigned to each robot in a way that one of them dominates the other. The assignment of aggression level is done even before they start their spatial navigation. The dominant robot gains access to the resource while the non-dominant robot submits to its opponent as soon as an encounter takes place. Figure 3(c) shows this.

Cutting your Losses: Some amount of memory of local task performance is added to the rational aggression method. When a robot meets an opponent inside the girdle, it displays its level of aggression for at most $\phi$ attempts. At the same time it measures the lose or gain in the shared space distance from the time it starts its aggressive display and remembers the cost it incurs during this display. In our current problem domain cost can be considered as the time spent in arbitration. Figure 3(d) shows this mechanism.

Random walk: As soon as a robot encounters an opponent, it backs a random distance. It then moves forward and if the opponent is still in the girdle, it again moves back by a random distance. The opponent also follows the same protocol. Eventually one of the robots is pushed out of the girdle. Figure 3(b) illustrates this mechanism. 


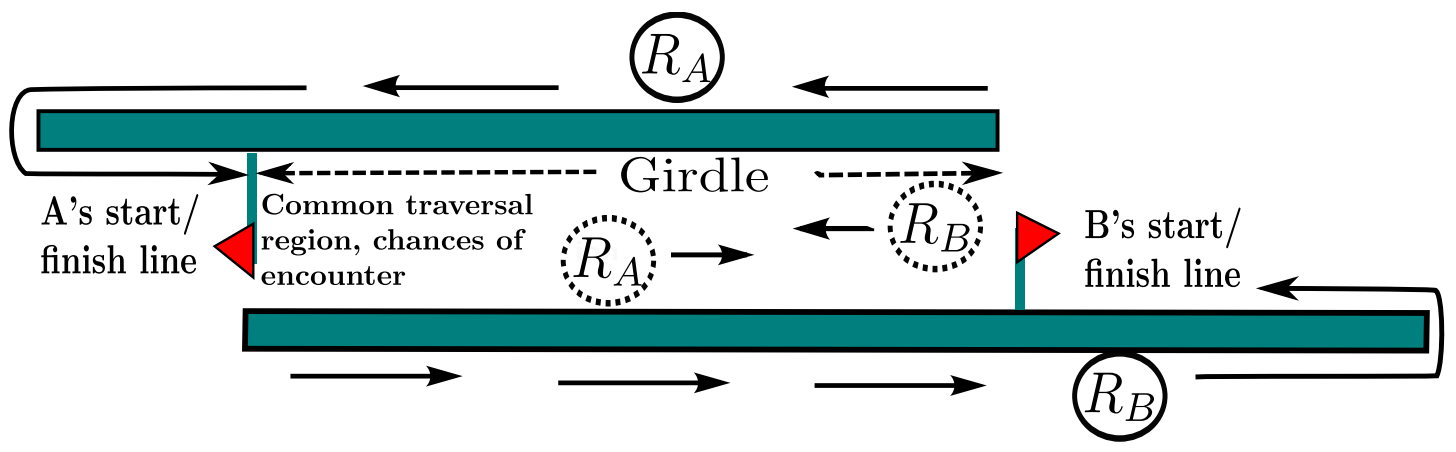

Fig. 1. Sketch of the task environment navigated by the robots. The arrows show the direction of motion of the robots $R_{A}$ and $R_{B}$. The flags mark the start and end points for completing one traversal tasks by each robot. The common traversal region of the robots is a narrow corridor, which we term as the "girdle". Spatial interference takes place when both $R_{A}$ and $R_{B}$ attempt to move through the girdle from the opposite directions in the same time.

C. Taxonomy of Spatial Conflict Resolution Methods

We propose a taxonomy of spatial conflict resolution methods based on the following axes:-

- Dynamic (D) vs. Static (S): An arbitration method is static if and only if it does not employ information about a particular encounter to resolve that resource conflict.

Arbitration Method is "Static", if $\forall x_{A}, x_{A}^{\prime}, p\left(x_{A}\right) \equiv p\left(x_{A}^{\prime}\right)$,

Arbitration Method is "Dynamic", otherwise.

- Deterministic (DET) vs. Probabilistic (PROB): A method is deterministic if and only if, given the same scenario, the resource is always awarded to the same agent. 


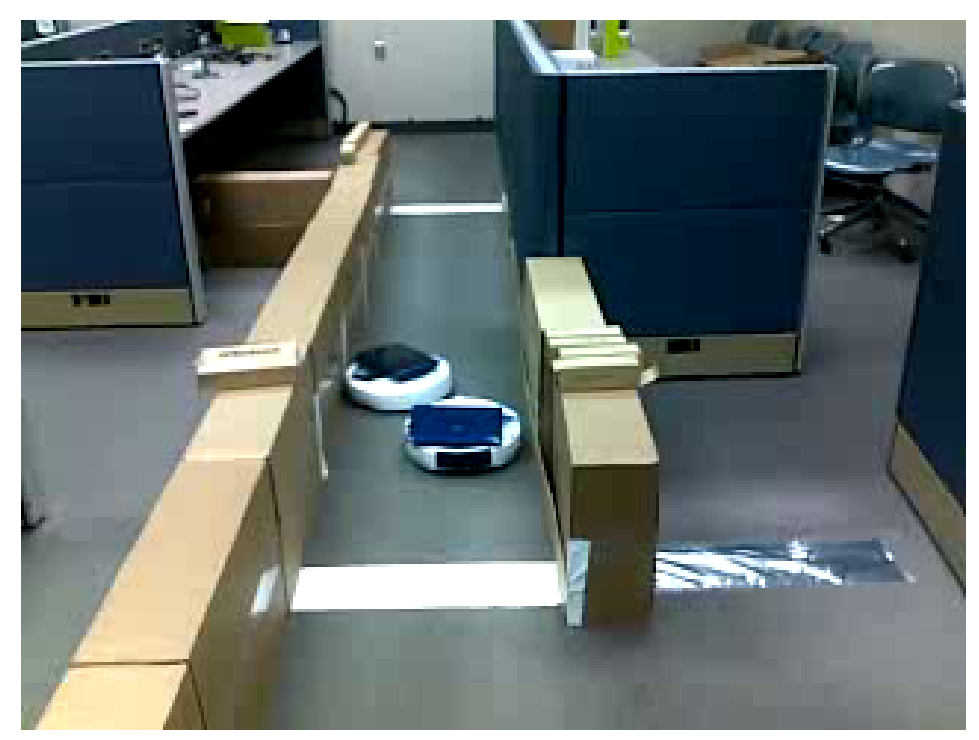

Fig. 2. Physical robots traversing a narrow corridor at the same time results in spatial interference.

\section{Arbitration Method is "Deterministic", if $p\left(x_{A}\right)=(1,0)$, Arbitration Method is "Probabilistic", otherwise.}

- High outcome accuracy (HOA) vs. Low outcome accuracy (LOA): The former has higher probability of selecting the rational winner. A rational winner is the robot which has put in more local investment in crossing a shared space and hence gets the right of way in the event of an encounter. Such an arbitration mechanism would be termed as rational. Consider an ideal rational arbitration method, where robot $R_{A}$ wins the interaction if it is at least half way down the girdle, otherwise it loses. We assume that for $x=0.5$, the boundary condition when the encounter is exactly at the center, $R_{A}$ can either win or lose. We denote the probability of $R_{A}$ being the winner of such an ideal interaction by a 

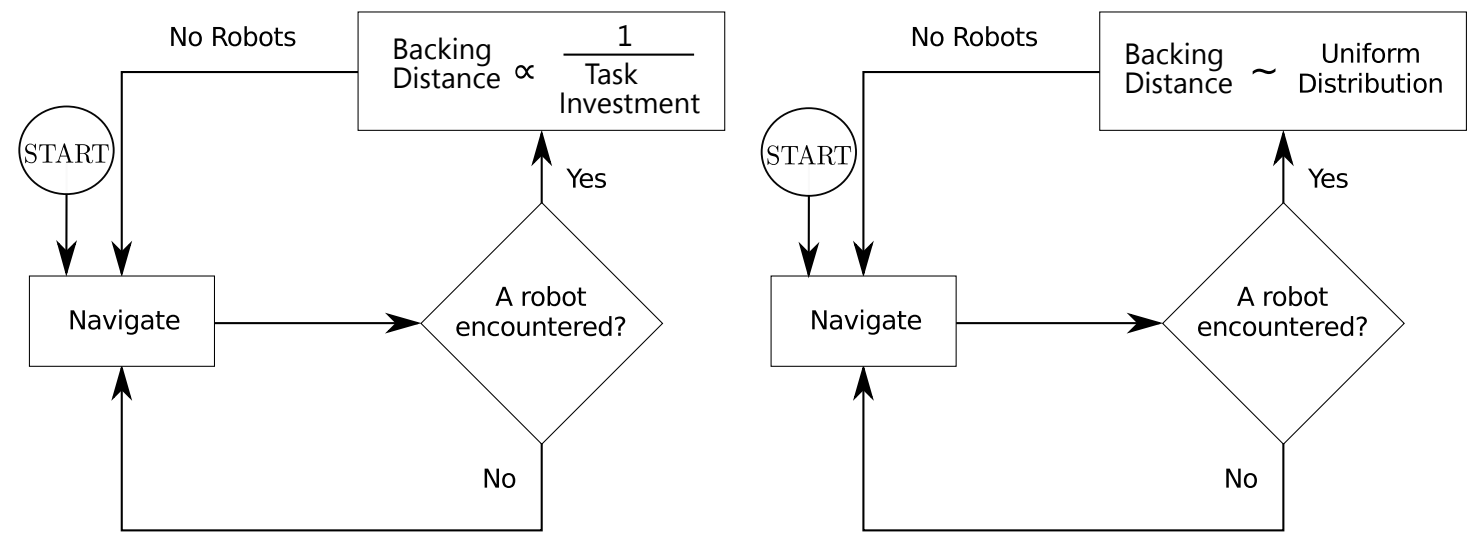

(a) Aggression-based arbitration.

(b) Random walk arbitration.

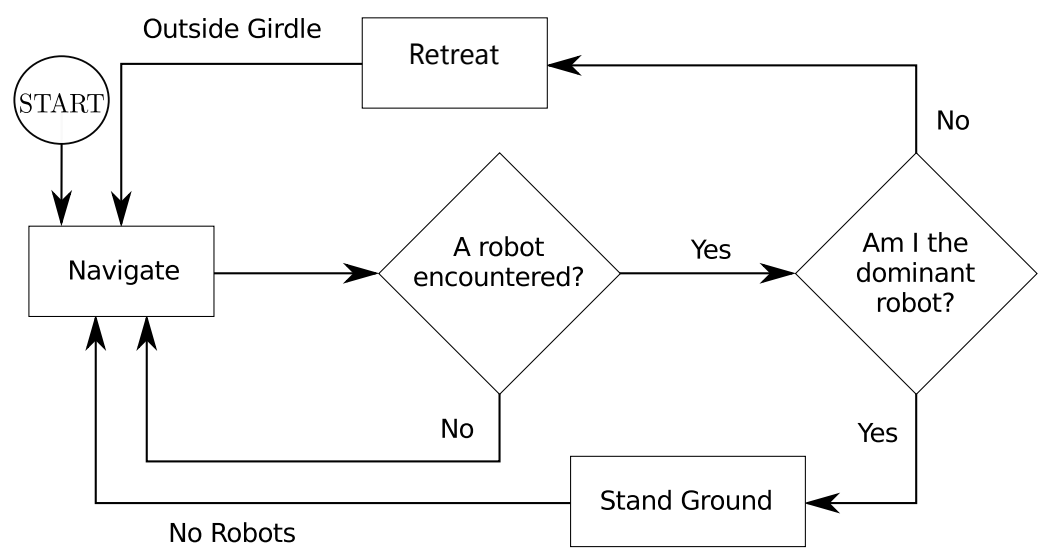

(c) Fixed dominance hierarchy.

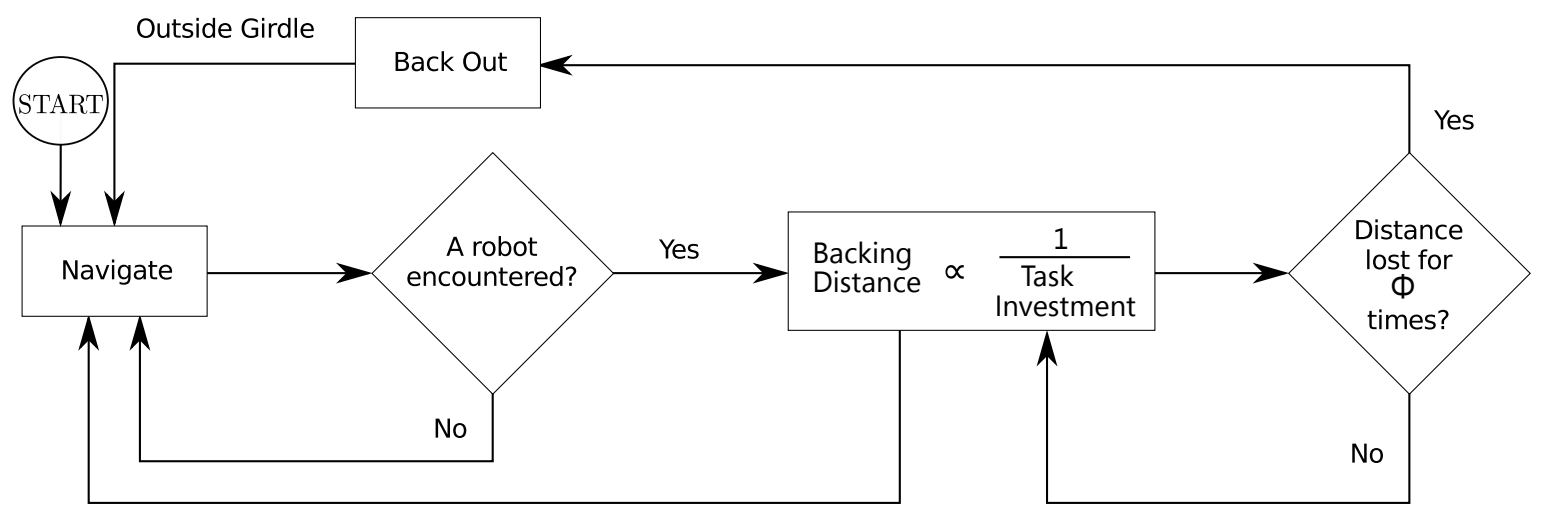

No Robots

(d) Cutting one's losses.

Fig. 3. Four different arbitration mechanisms. 
probability distribution $P(x)$ as follows:

$$
P(x)= \begin{cases}1 & \text { if } 0 \leq x<0.5 \\ 0.5 & \text { if } x=0.5 \\ 0 & \text { if } 0.5<x \leq 1 .\end{cases}
$$

Here $x$ is the distance measured from $\mathrm{R}_{A}$ 's girdle entry point. We quantify the outcome accuracy of a non-ideal arbitration method by comparing its probability distribution with that of the ideal method. Let $Q(x)$ denote the probability distribution of a non-ideal arbitration method. Then the extent to which a non-ideal arbitration differs from ideal arbitration is:

$$
\int_{0}^{1}|P(x)-Q(x)| d x .
$$

We call this the "score" of an arbitration method and it measures how close a non-ideal arbitration method is to an ideal arbitration method. A score greater than 0.5 denotes that the arbitration method has high outcome accuracy.

Arbitration Method is "HOA", if score $>0.5$ Arbitration Method is "LOA", otherwise.

Below are the calculated scores of a few non-ideal arbitration methods:-

1. Vaughan's random arbitration - In Vaughan's random arbitration at every encounter the winner is determined by the flip of a coin. Thus every agent has a probability of 0.5 to win an encounter with respect to its position of encounter. Hence we have:

$$
Q(x)=0.5, \quad 0 \leq x \leq 1 .
$$


Using equation 3.1 and 3.2 , the following score is obtained:

$$
\text { Score }=0.5 \text {. }
$$

2. Aggressive interaction-The empirical data from physical robot experiments were used to obtain the score for an aggressive interaction. The probability with which a robot wins an encounter with respect to its position of encounter in a normalized girdle length is fitted to the following equation and is shown by the green curve in Figure 4 .

$$
Q(x)=\frac{1}{1+e^{-55.4(x-.510)}}, \quad 0 \leq x \leq 1 .
$$

Using equation 3.1 and 3.3 we obtain the following score:

$$
\text { Score }=0.97368 .
$$

3. Random Walk - The random walk arbitration was simulated on a girdle space of 5 meters, sampled at 1000 different positions. These positions served as encounter positions for the robots. Figure 5 shows the probability of a robot winning an encounter with respect to its encounter position. The plotted data lie in a straight line. Hence we can say that:

$$
Q(x) \propto x \text {, where } x \text { is the position of encounter. }
$$

One simplifying assumption while generating this plot is the absence of noise in the simulation model. We purposely simulated random walk arbitration rather than physical robot data, because the extended duration of random arbitration in physical robot experiments makes substantial data collection impractical. Figure 6 shows the corresponding time taken to re- 


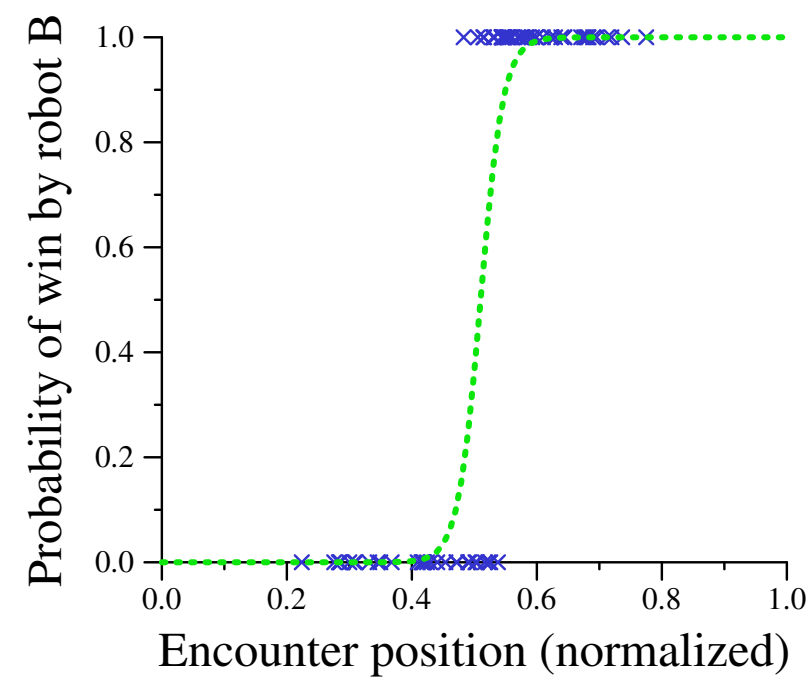

Fig. 4. Robot $R_{B}$ 's wins with respect to the position of encounter is plotted. The data was obtained by running physical robot experiments for different girdle lengths. The vertical axis is the probability that $R_{B}$ wins the encounter. Thus the vertical axis value is 1 when $R_{B}$ wins an encounter and is 0 when it loses. While plotting, all girdle lengths are normalized across a $(0,1]$ scale. We then obtain the best fit of this data as shown by the green curve. 


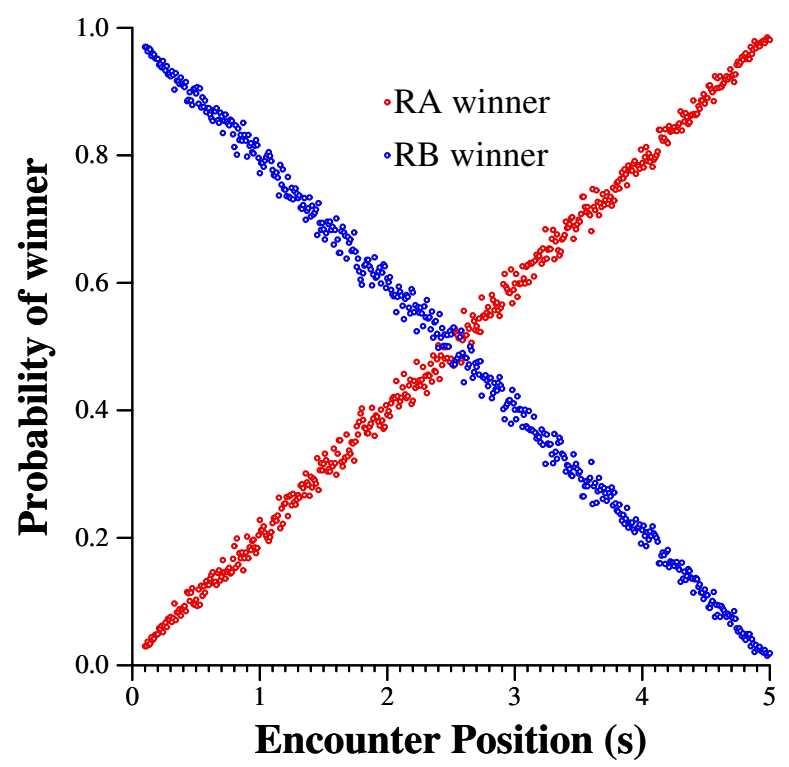

Fig. 5. The random walk arbitration was done over 1000 different positions inside the girdle of length 5meters. The fit of the probability of winner with respect to the encounter position was found to be linear.

solve each of these 1000 encounters. We see that the duration of arbitration increases when encounter is at the center of the girdle.

The accuracy of the random walk arbitration is obtained as follows:

$$
\begin{gathered}
\int_{0}^{1}|P(x)-Q(x)| d x=\int_{0}^{0.5}(1-x) d x+\int_{0.5}^{0.5}(0.5-x) d x+\int_{0.5}^{1}(1-x) d x \\
=0.375+0+.125=0.5
\end{gathered}
$$

- Costly (HCOST) vs. cheap (LCOST): Time, energy and other resources may be involved in an arbitration mechanism. Their utility depends on the comparative saving and/or trade-off of these costs. The cost of an arbitration mechanism increases as it spends more and more time in arbitrating the resource and determining the winner of the encounter. Clearly all static arbitration mechanisms waste almost no time in resource arbitration since the winner of any encounter 


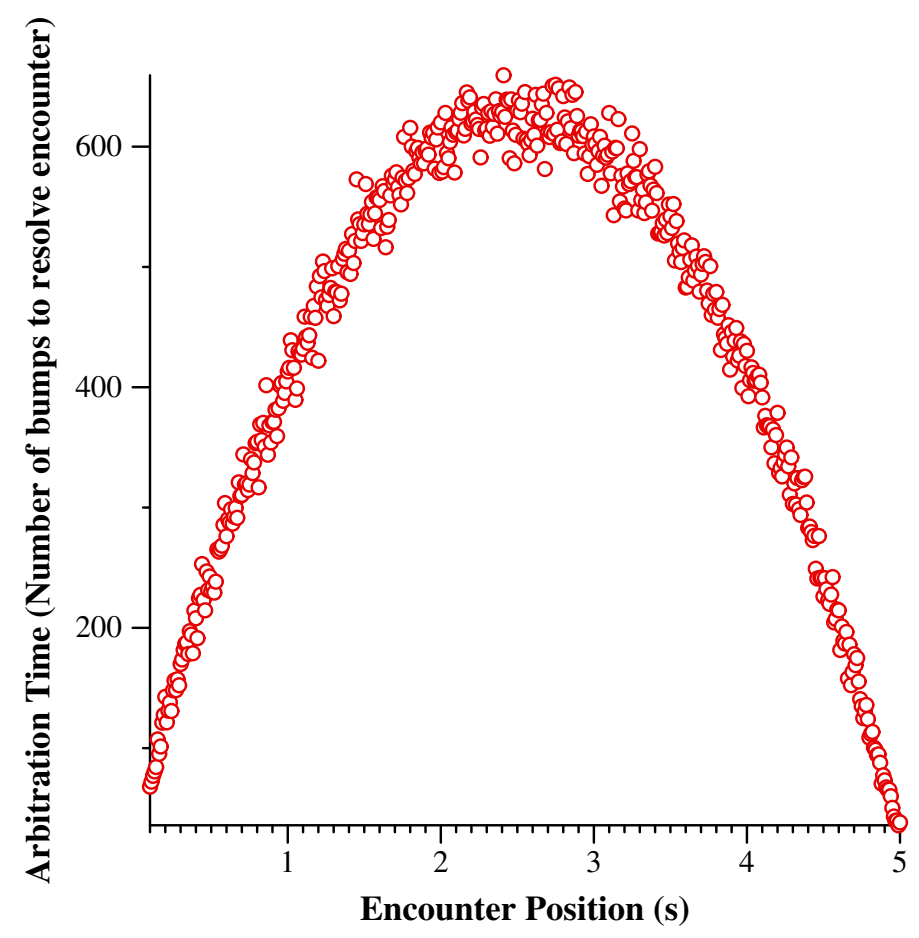

Fig. 6. Time taken for random walk arbitration with respect to the position of encounter. 
Table I. Summary of arbitration methods

\begin{tabular}{|l|l|}
\hline Arbitration Method & Classification \\
\hline Linear dominance hierarchy & S-DET-LOA-LCOST \\
\hline Vaughan's random aggression & S-PROB-LOA-LCOST \\
\hline Random walk & D-PROB-LOA-HCOST \\
\hline Vaughan's Personal space & D-DET-HOA-HCOST \\
\hline Rational aggression & D-PROB-HOA-HCOST \\
\hline Cutting your losses & D-PROB-HOA-LCOST \\
\hline
\end{tabular}

is pre-determined. On the other hand dynamic arbitration mechanisms where there is repeated interactions without any assessment of the cost of the interaction needs long time for resource arbitration, resulting in high arbitration cost. In case of "Cutting your Losses" dynamic arbitration mechanism there is an assessment involved in each interaction and an earlier arbitration decision based on a fewer interactions is less costly though it might not have very high outcome accuracy.

Hence the different arbitration methods can be summarized by a quadruplet as shown in Table I.

We also present the calculation of the cost of an interference pattern for any arbitration method later in Chapter VIII. 


\section{CHAPTER IV}

\section{EXPERIMENTAL DESIGN}

This chapter presents details of physical robot experiments done to induce spatial interference. The data collected from real robot experiments gave way to the design of a simulator which enabled the study of the arbitration mechanisms over a range of environmental parameters.

\section{A. Physical Robot Interference}

Spatial interference on physical robots was imposed by making them navigate through an environment as shown in Figure 9. Interference is induced when two iRobot Creates, $\mathrm{R}_{A}$ and $\mathrm{R}_{B}, \sim 33 \mathrm{~cm}$ in diameter attempt to cross a girdle $\sim 53 \mathrm{~cm}$ wide from opposite directions as shown by the arrows in Figure $9 . \mathrm{R}_{B}$ 's transportation task length is almost half that of $\mathrm{R}_{A} \cdot \mathrm{R}_{A}$ does 10 traversals, while $\mathrm{R}_{B}$ covers 20 . These numbers are purposely assigned so as to avoid situations where the robot performing the shorter task finishes all its trips while the other one continues to traverse an encounter-free region. Each robot detects the presence of an opponent inside the girdle with its left bumper sensor. Right bumper and wall following sensors are used to wall follow through the environment. The girdle is marked by placing white colored strips, while corners are marked by reflective black strips as shown in Figure 9. The strength of the cliff sensors return a value depending on the color of the floor the robot moves over, enabling the robot detect the start of the corridor or a corner. These built-in sensors are the only ones employed.

Player robot controller running on Linux PC is used. The client programs were written in C. The following arbitration mechanisms as mentioned in the previous chapter was implemented using this minimal set of sensors:- 


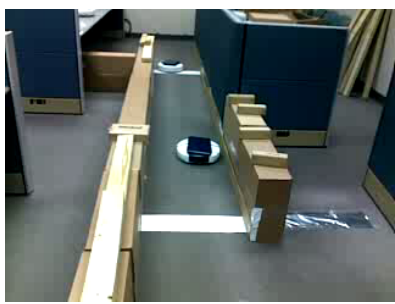

(a)

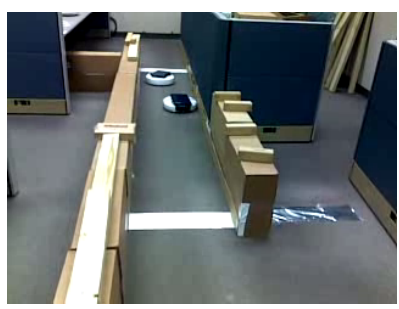

(d)

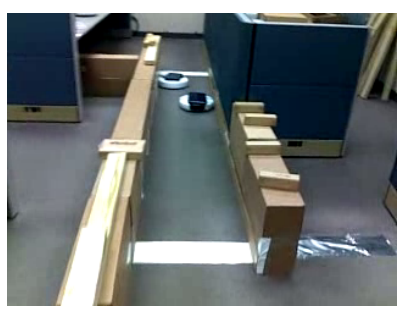

(g)

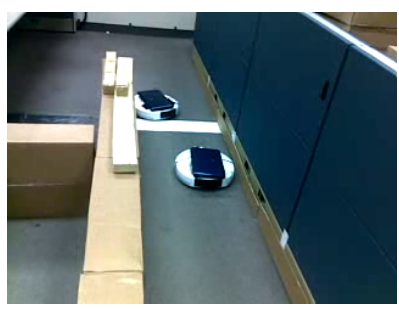

(j)

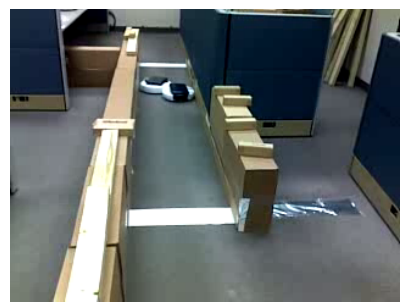

(b)

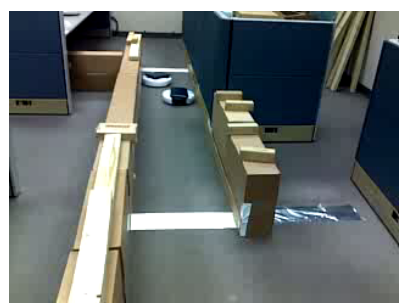

(e)

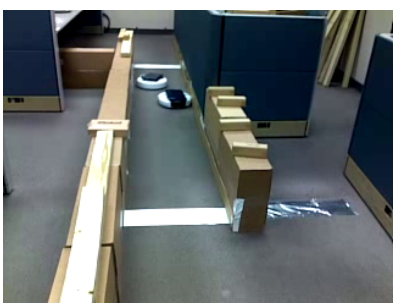

(c)

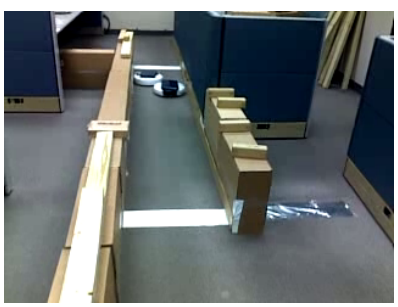

(f)

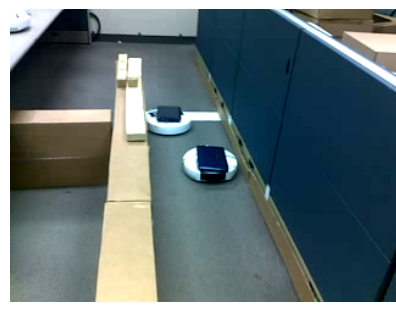

(h)

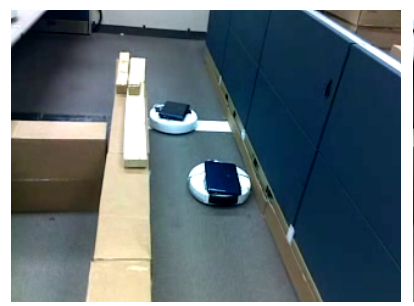

(k) (i)
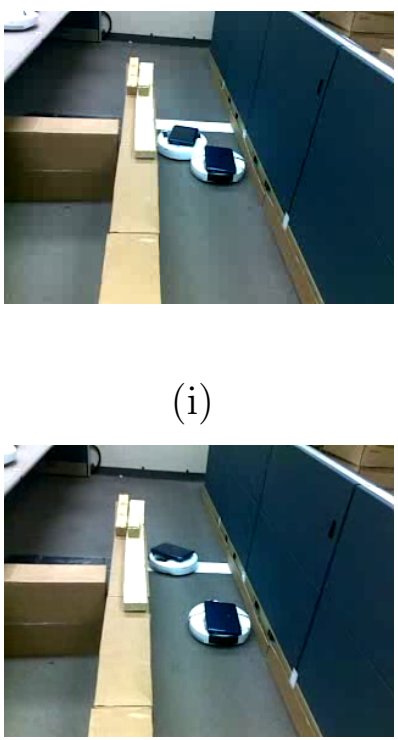

(1)

Fig. 7. Interference resolution in physical robots by aggressive interactions. (a). Two robots approach each other in a narrow girdle, (b). Two robots bump into each other (spatial interference), (c), (d). Each robot backs by a distance inversely proportional to its local investment inside the girdle, (e). The robots move forward after they backing, (f). They bump into each other again, (g), (h). They back proportional to their local investment so far, (i). They move forward and bump into each other again, $(\mathrm{j}),(\mathrm{k}),(\mathrm{l})$. The losing robot is pushed out of the girdle giving way to the winner. 


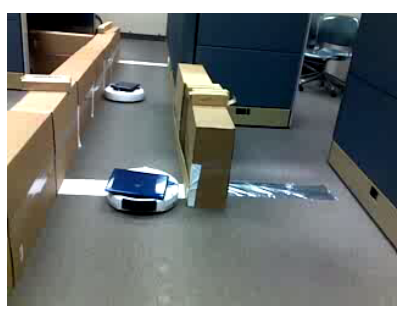

(a)

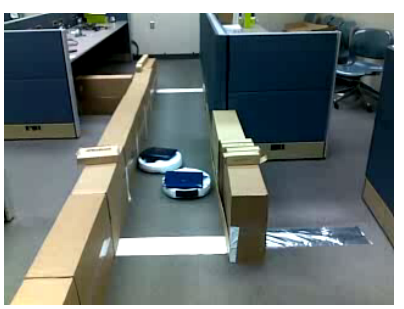

(d)

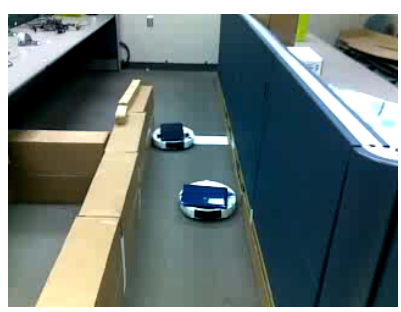

$(\mathrm{g})$
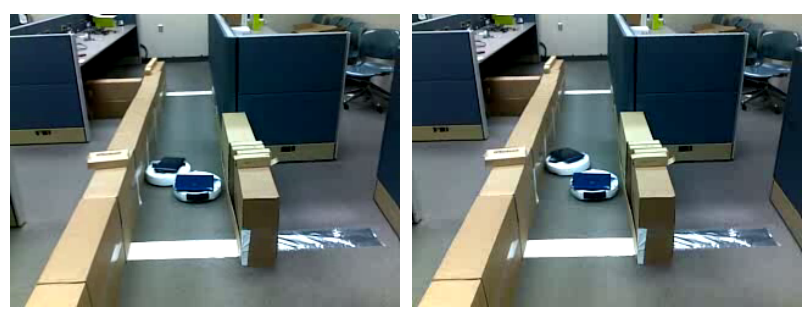

(b)

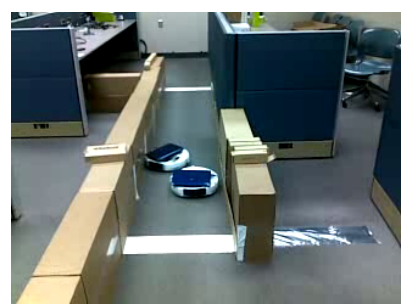

(e)

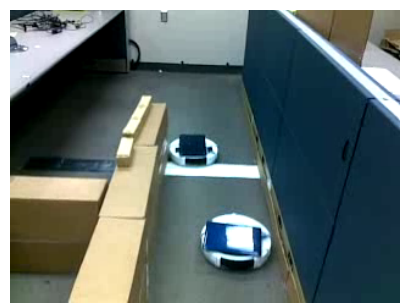

(h) (c)

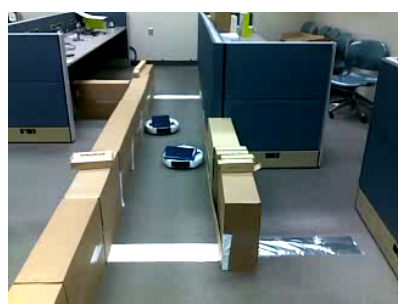

(f)

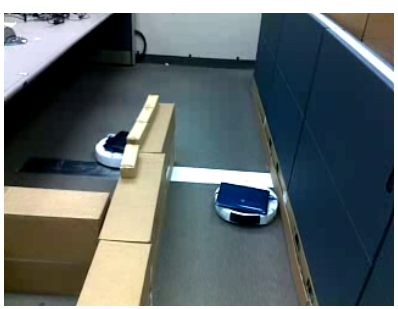

(i)

Fig. 8. Interference resolution in physical robots by follow of linear dominance hierarchy. (a). Two robots approach each other in a narrow girdle, (b). Two robots bump into each other (spatial interference), (c), (d), (e). The dominant robot stands ground and the dominated robot turns around to move out of the girdle, (f), (g), (h). The dominated robot leaves the girdle giving right of way to the dominator. 


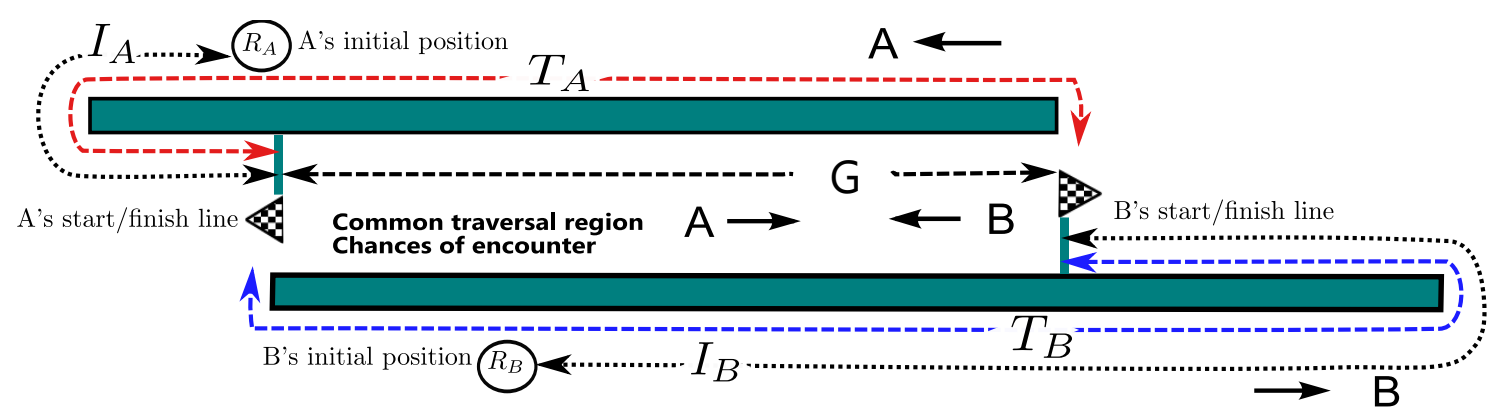

Fig. 9. Task Environment navigated by the robots. The initial positions correspond to the positions from where the robots start their traversal for the first time. The flags mark the start and end points for completing one traversal. For the very first traversal, $I_{A}$ and $I_{B}$ are the initial distances from the start point. A single traversal includes traveling in the non-shared region, (marked as $T_{A}$ and $T_{B}$ for robots $R_{A}$ and $R_{B}$ respectively) and moving in the shared common space (termed as the girdle and marked as $G$ ).

1. Rational aggression based on local task investment.

2. Linear dominance hierarchy.

3. Cutting your losses.

4. Random walk.

Figure 7 shows the snapshot of an aggressive interaction while Figure 8 shows the same for a dominance based arbitration.

\section{B. Simulated Interference}

Based on physical robot data collected from the above setup, a custom simulator was designed to model dominance and aggressive robot interactions for different girdle lengths, across a range of task ratios. The purpose was to perform a macroscopic study of the navigation environment. The simulator used the following information from physical robot data to generate the dominance and aggressive interaction models. 
1. Characteristics of the task behavior outside of the shared space. This is called the task model.

2. Characteristics of the task behavior inside of the shared space when no interfering robot is present.

3. Characteristics of the robot's behavior inside the shared space when two robots interact. This is the fight model.

\section{Models of motion without interference}

Both (1.) and (2.) are treated in a unified way because in this particular problem domain the robot follows a wall with essentially the same speed whether outside or inside the girdle when no other robot is encountered. Figure 10 shows that there is comparatively little variation in the task performance time in both the cases (both are for different sized tasks, but have standard deviation, $\sigma \approx 1.3$ once egregious outliers have been removed.) The distance plot for unhindered movements across the shared space is shown in Figure 11. The variation appears to be invariant of the magnitude of the space.

(1.) and (2.) are modeled by fitting a velocity to the recorded data: $v=0.05 \mathrm{~m} / \mathrm{s}$, but with a time that includes additive noise with $\sigma=1.3$ seconds. Figures 12 and 13 shows the qualitative correspondence between data collected and generated.

\section{Model of interference}

Two aspects need to be considered in arbitration models. First, the decision as to which robot is the "victor" and gains right of way. The second (which may depend on the first) is the time taken to exit the shared space. Both the dominance and the aggression model is simulated to analyze the influence of resource and task properties 


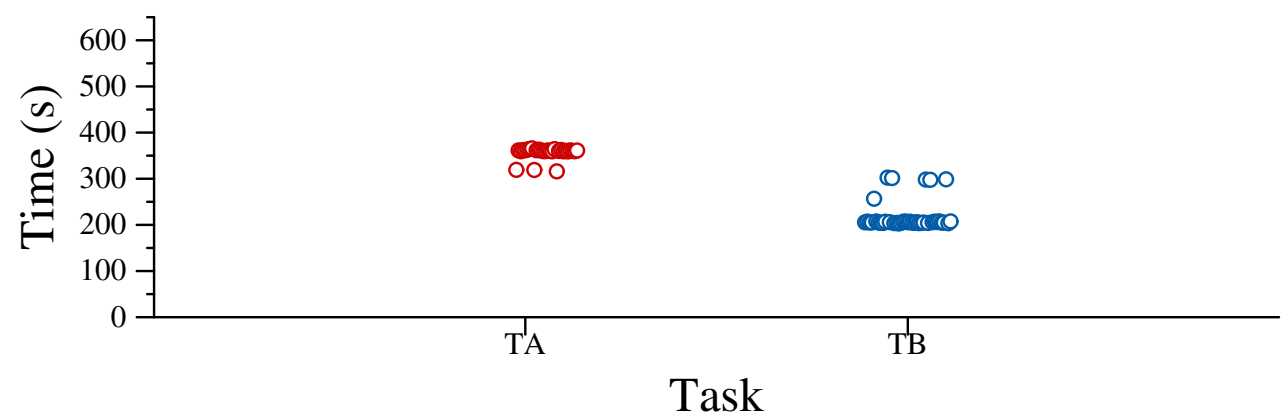

Fig. 10. Task completion times.

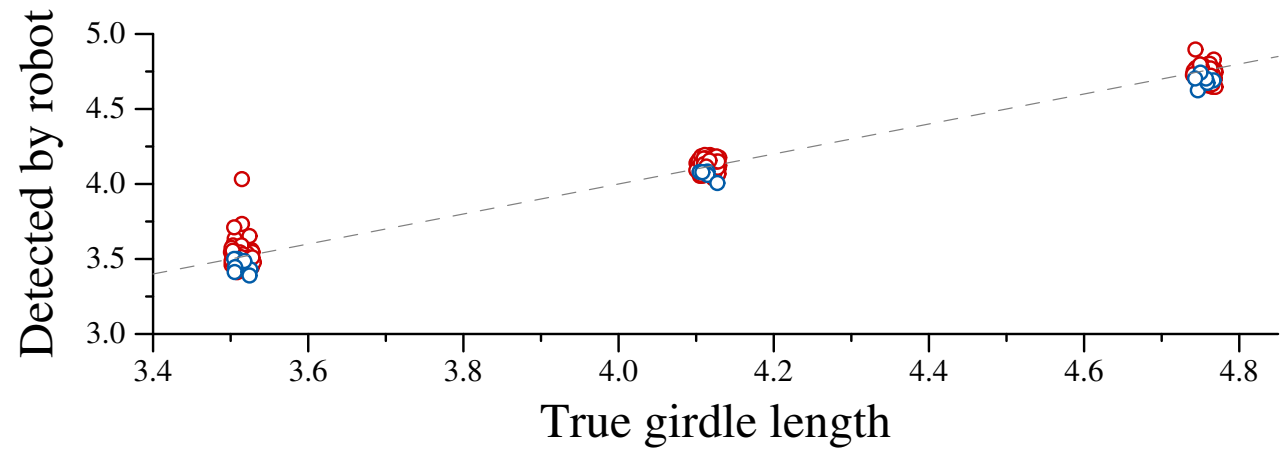

Fig. 11. Measured versus true girdle movements. 


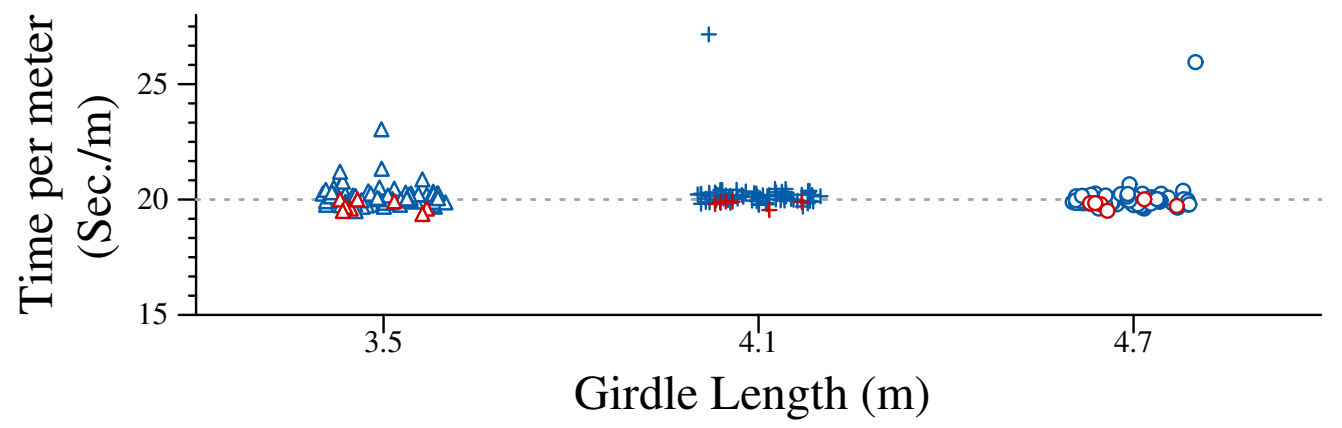

Fig. 12. Physical robot data.

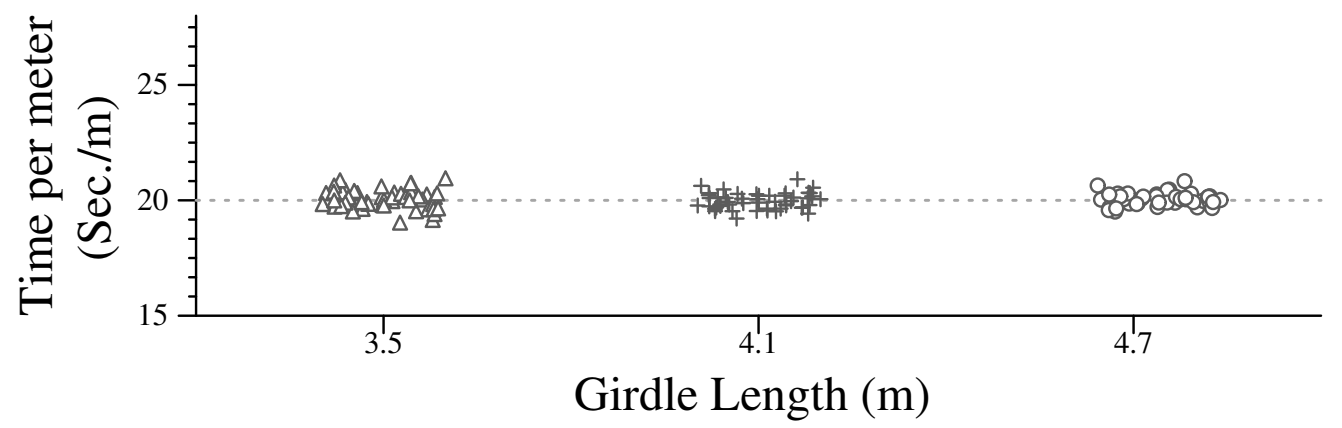

Fig. 13. Simulated using the parameters described above. 


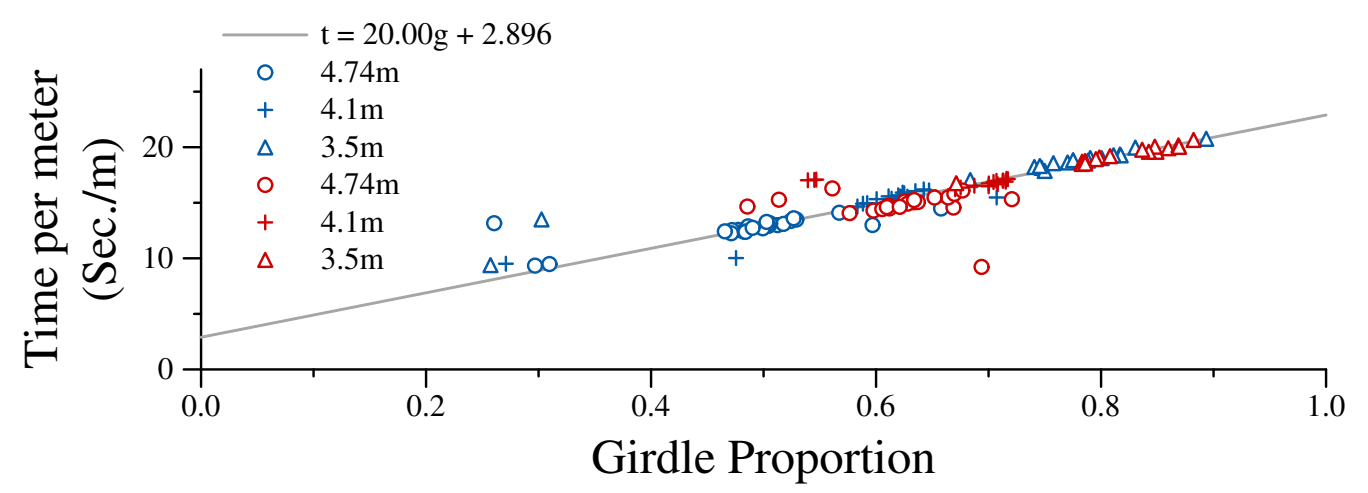

Fig. 14. Time (length normalized) to resolve an interaction with the dominance model.

of aggressive interaction. For each of these models simulations were run by varying the girdle lengths from 10 to 150 and for each girdle length task lengths covered by each robot was varied from 15 to 150. Data from each of these runs were collected and analyzed. The following section discusses each of these models and the analysis of the collected simulation data.

a. Dominance model

A single robot is marked as "dominator" and always wins the aggressive interaction. Figure 14 shows the time of a dominance fight model, along with a straight line fit. Figure 15 shows the residual and a normal distribution that describes all but extreme outliers. The result (with coefficients correct to four significant digits) is time $=20.00$ gird $\%+2.896$, along with additive zero mean Gaussian noise with $\sigma=0.320$. Figure 16 shows data generated using this approach. 


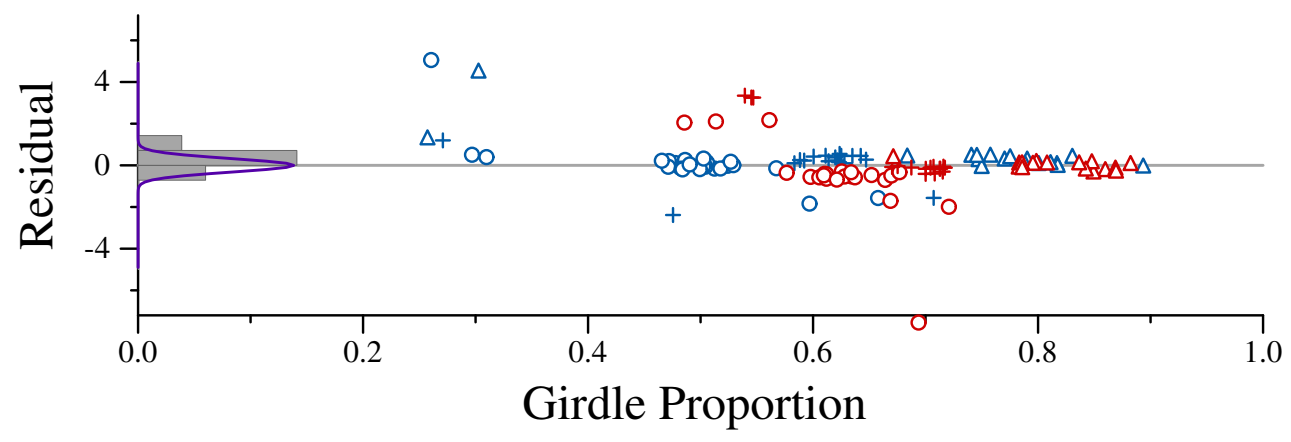

Fig. 15. Noise in the length normalized time model $(\sigma=0.320)$.

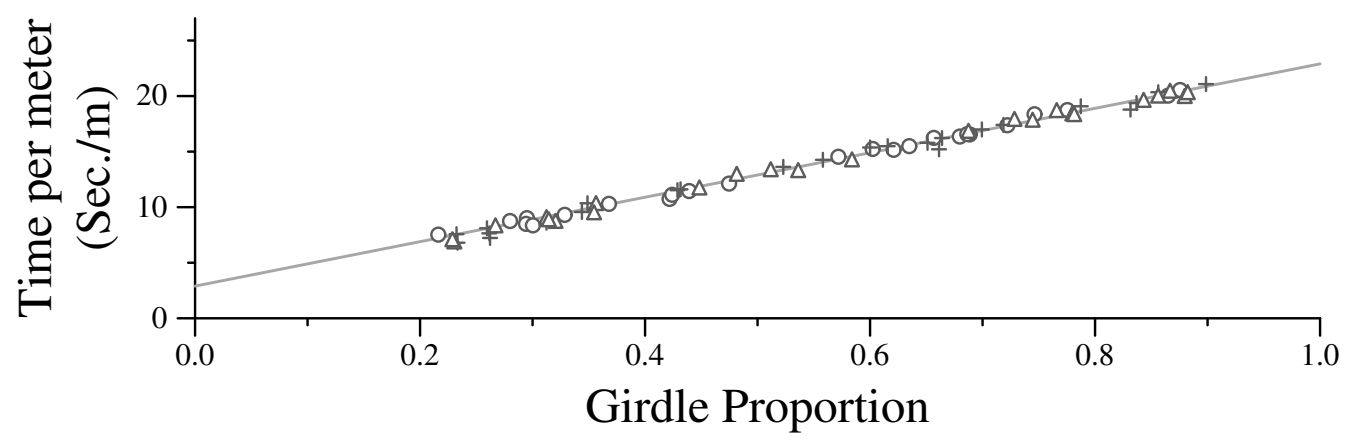

Fig. 16. Simulated length normalized time for dominance interactions . 


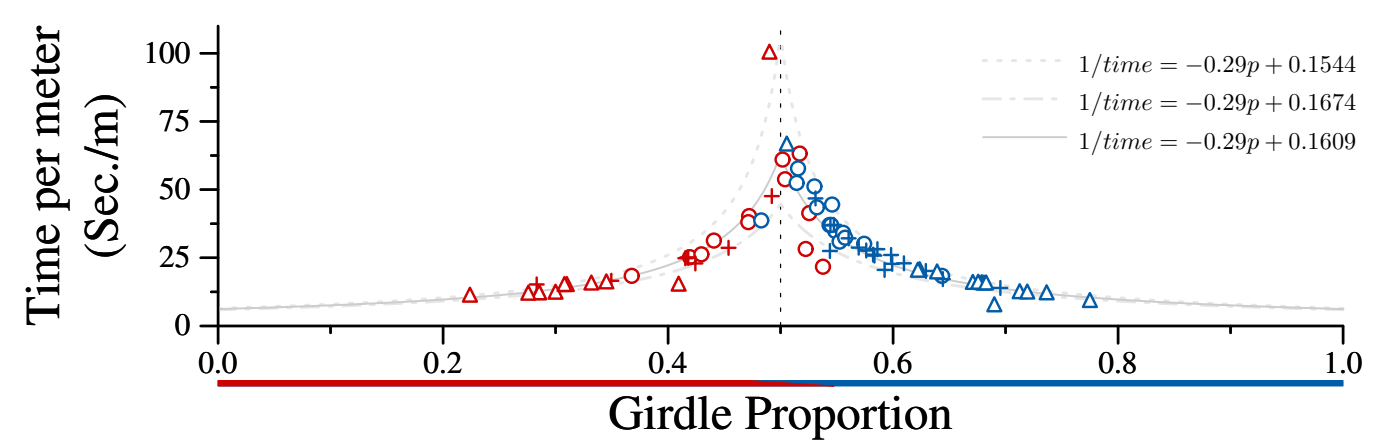

Fig. 17. Time (length normalized) to resolve an interaction with the aggression fight model.

b. Aggression model

In this model, each robot displays its aggression levels based on the local investment made by the robots [3]. The data collected from robot experiments across three girdle lengths were analyzed (Figure 17). By normalizing the data by the length of the girdle and taking the ratio of robot 1 to robot 2 victories, a linear fit was obtained. The following expression for the probability of the first robot (marked in red) winning is produced:

$$
\begin{gathered}
\operatorname{Pr}\left({\text { Winner } \left.=\text { Robot }_{1} \mid \text { Girdle }_{P} \text { Os }=x\right)}^{1}\right. \\
= \begin{cases}\frac{0.54750-x}{0.075} & \text { if } x \leq 0.4725 \\
0 & \text { if } x>0.54750,\end{cases}
\end{gathered}
$$

This is shown as the colored bar along the bottom of Figure 17.

The time that aggressive fight takes (normalized by girdle length, which must be 


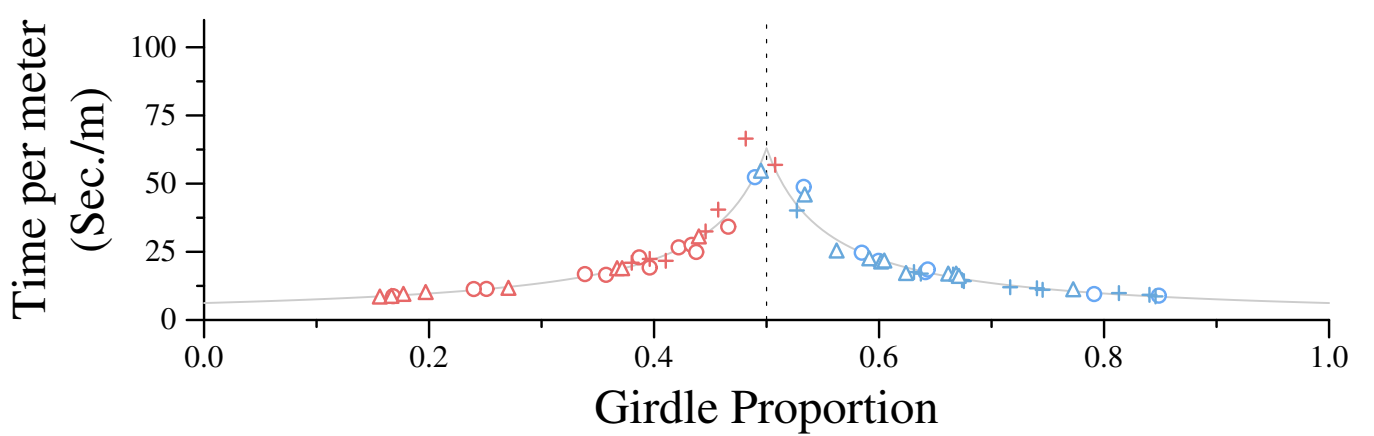

Fig. 18. Simulated length normalized time for aggressive fights.

performed due to the mechanics of the fight procedure) is described by a good fit to the following line:

$$
\frac{1}{\text { time }}=-0.29 \text { gird } \%+0.1609
$$

The figure also illustrates that the noise can be reasonably represented by uniformly sampling between the lines:

$$
\frac{1}{\text { time }}=-0.29 \text { gird } \%+0.1609 \pm 0.0065
$$

Figure 18 shows the result of the simulated aggressive fights. 


\section{CHAPTER V}

\section{COMPARATIVE STUDY OF INTERFERENCE MODELS}

In this chapter we show the results from our physical robot experiments and draw comparisons among the different arbitration methods. Our results refute the previous claim in literature that dynamic aggressive interaction is always better than following a fixed dominance hierarchy. Among others this is one of the contributions of this thesis work.

\section{A. Aggressive Interaction and Linear Dominance}

Both these models were executed in environments with different girdle lengths. The aim is to assess the role the shared resource plays on arbitration outcomes.

1. Varying Girdle Length - We empirically found that in a transportation environment as in Figure 9, when the ratio of individual task times of $\mathrm{R}_{A}$ and $\mathrm{R}_{B}$ is $25: 38$ it results in substantial interference. We purposely chose an interference ratio where there is frequent interference, in order to draw meaningful comparisons between the different arbitration mechanisms.

From Figure 19 we see that the utility of aggressive interaction is reduced when both robots have large, almost equal aggression levels, a phenomenon which happens when encounters are at the center of a large enough girdle. Following a dominance hierarchy proves to be a better arbitration method in such a scenario. However for encounters at the ends of the girdle, the short aggressive interactions coupled with the ability to produce a rational winner makes dynamic aggression based arbitration beneficial over dominance. [1] provides an instance where dynamic choice of aggression level proves no better than random 


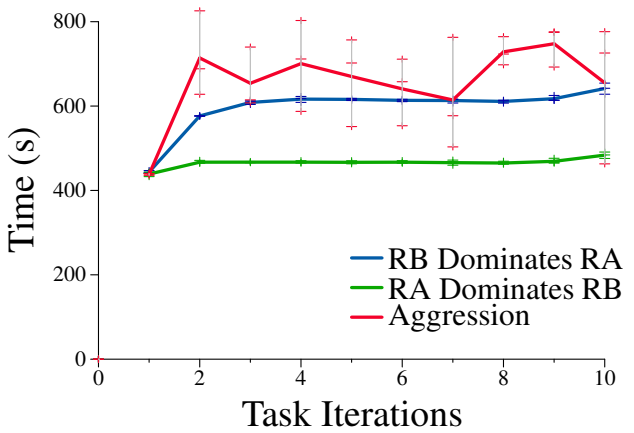

(a) $\mathrm{R}_{A}$, Girdle $=4.7 \mathrm{~m}$

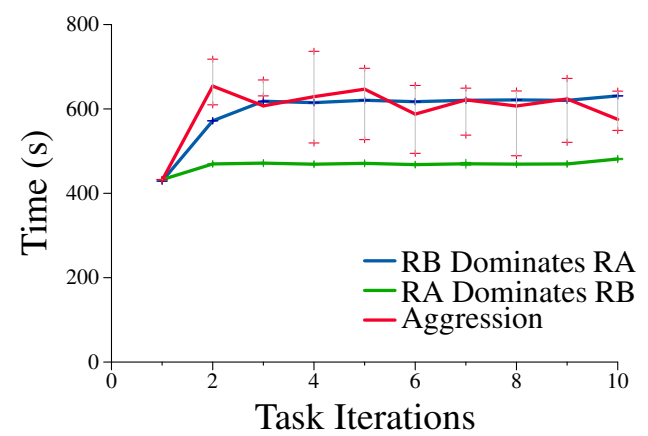

(c) $\mathrm{R}_{A}$, Girdle $=4.1 \mathrm{~m}$

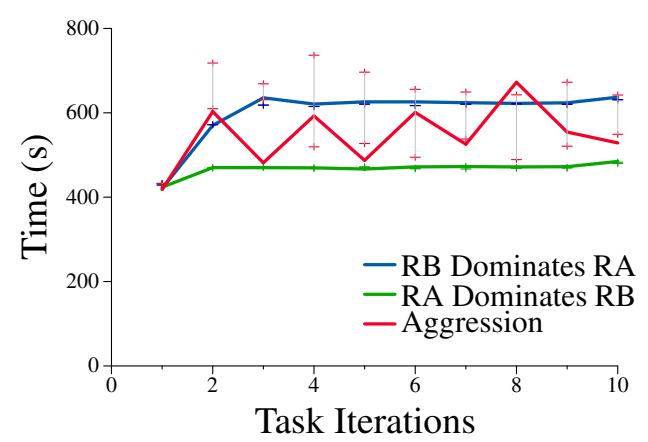

(e) $\mathrm{R}_{A}$, Girdle $=3.35 \mathrm{~m}$

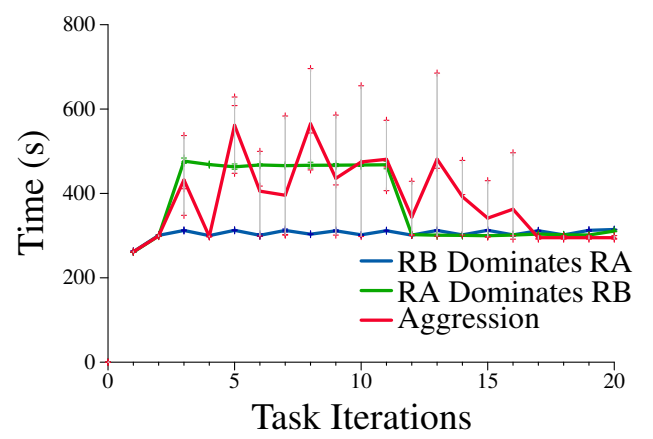

(b) $\mathrm{R}_{B}$, Girdle $=4.7 \mathrm{~m}$

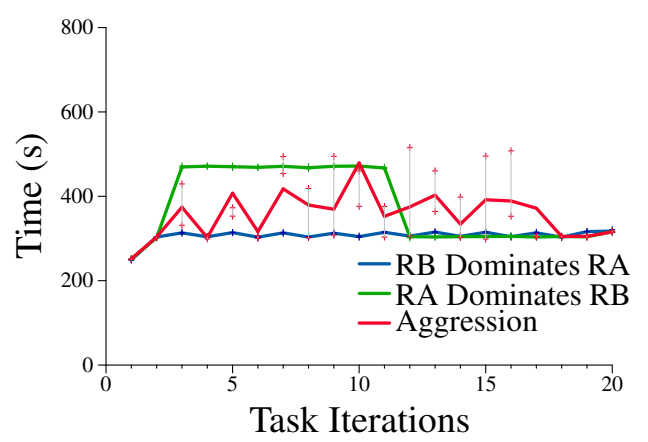

(d) $\mathrm{R}_{B}$, Girdle $=4.1 \mathrm{~m}$

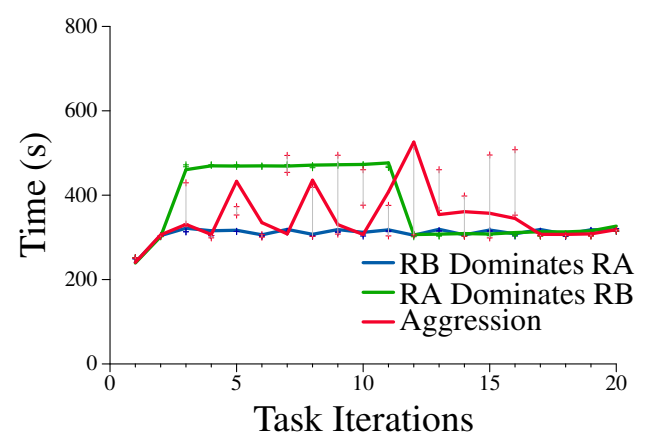

(f) $\mathrm{R}_{B}$, Girdle $=3.35 \mathrm{~m}$

Fig. 19. Average task times of $\mathrm{R}_{A}$ and $\mathrm{R}_{B}$ with varying girdle lengths, fixed task ratio 25:38. 

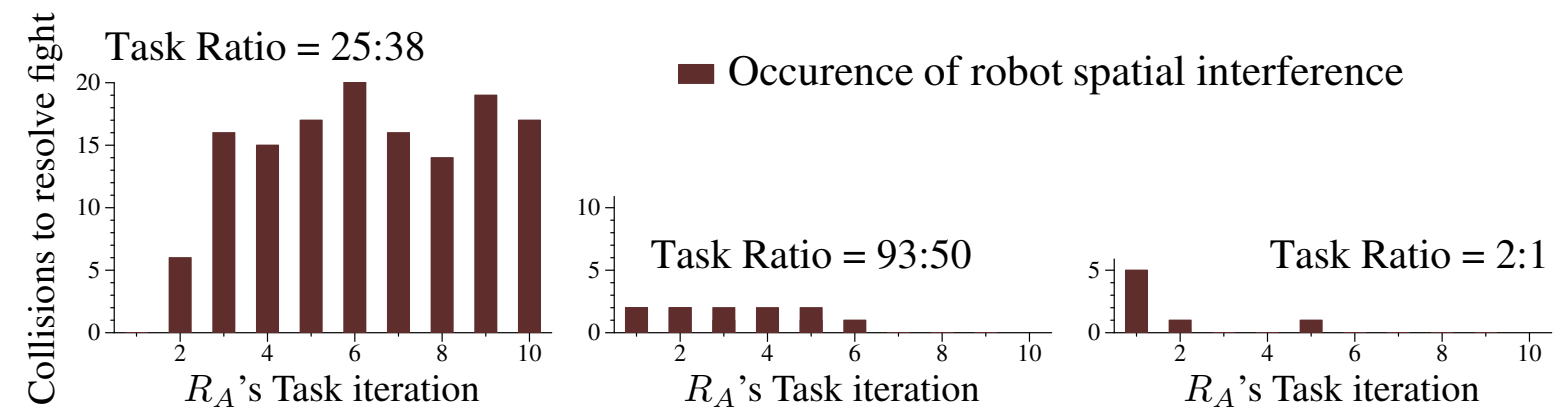

Fig. 20. Duration of aggressive interaction with varying task ratio.

selection of aggression (Vaughan's random aggression). In linear dominance the winner is pre-determined and is independent of encounter position. Random arbitration determines a winner of an encounter by the flip of a coin and is independent of the position of encounter. In other words for every coin flip, the winning robot is the dominant agent of that encounter and for an unbiased coin there is equal probability of either of the two agents to be picked up as the winner of an encounter. The two agents winning an encounter represents the two extremes of a linear dominance hierarchy. Thus the outcome of such a random mechanism is an average drawn from the outcomes of following either extremes of the linear dominance hierarchy, namely $R_{A}$ or $R_{B}$ being the dominator. Thus Vaughan's work in [1] shows that dynamic choice of aggression level is no better than following the average case of the fixed linear dominance hierarchy.

An important question is "how precisely can the outcome of the arbitration be predicted given the initial position of encounter?" If the aggression level is a function of the local investment then in case of equal local investments the noise in the robot's interactions breaks the symmetry and determines the winner. In such a scenario the winner might not be a perfectly rational winner. In Figure 17 
the mixed red and blue region near the center of the girdle (girdle proportion $\sim 0.5$ ) shows that there are situations when $\mathrm{R}_{A}$ 's local investment is slightly less than $\mathrm{R}_{B}$, but $\mathrm{R}_{A}$ wins or vice-versa. These are the few instances when the outcome of the aggressive encounter is neither particularly accurate nor rational and there is high cost involved, decreasing their utility in such situations. A low cost, less accurate dominance arbitration method will be more meaningful. In the next section we also present a new arbitration method which has the benefits of low cost as well as high outcome accuracy.

Moreover, in instances where encounters occur at girdle ends, the inaccuracy of dominance hurts only when the less dominant robot has higher local investment and still retreats. If the task ratios are carefully chosen, then such scenarios may occur only rarely, making dominance arbitration the superior arbitration model for such environments.

2. Varying Task Ratio - We examine how the properties of the task assigned to each agent influence aggressive encounters. This factor dictates the time when a robot starts its journey inside the girdle relative to the other and, in-turn, the initial position where they end up meeting. There is also a chance that they do not meet at all.

The variation of the duration of aggressive interactions as shown in Figure 20 indicates the importance of task structure in aggression based arbitration. Task structure is reexamined in further detail when we present our simulator results in the next chapter. 


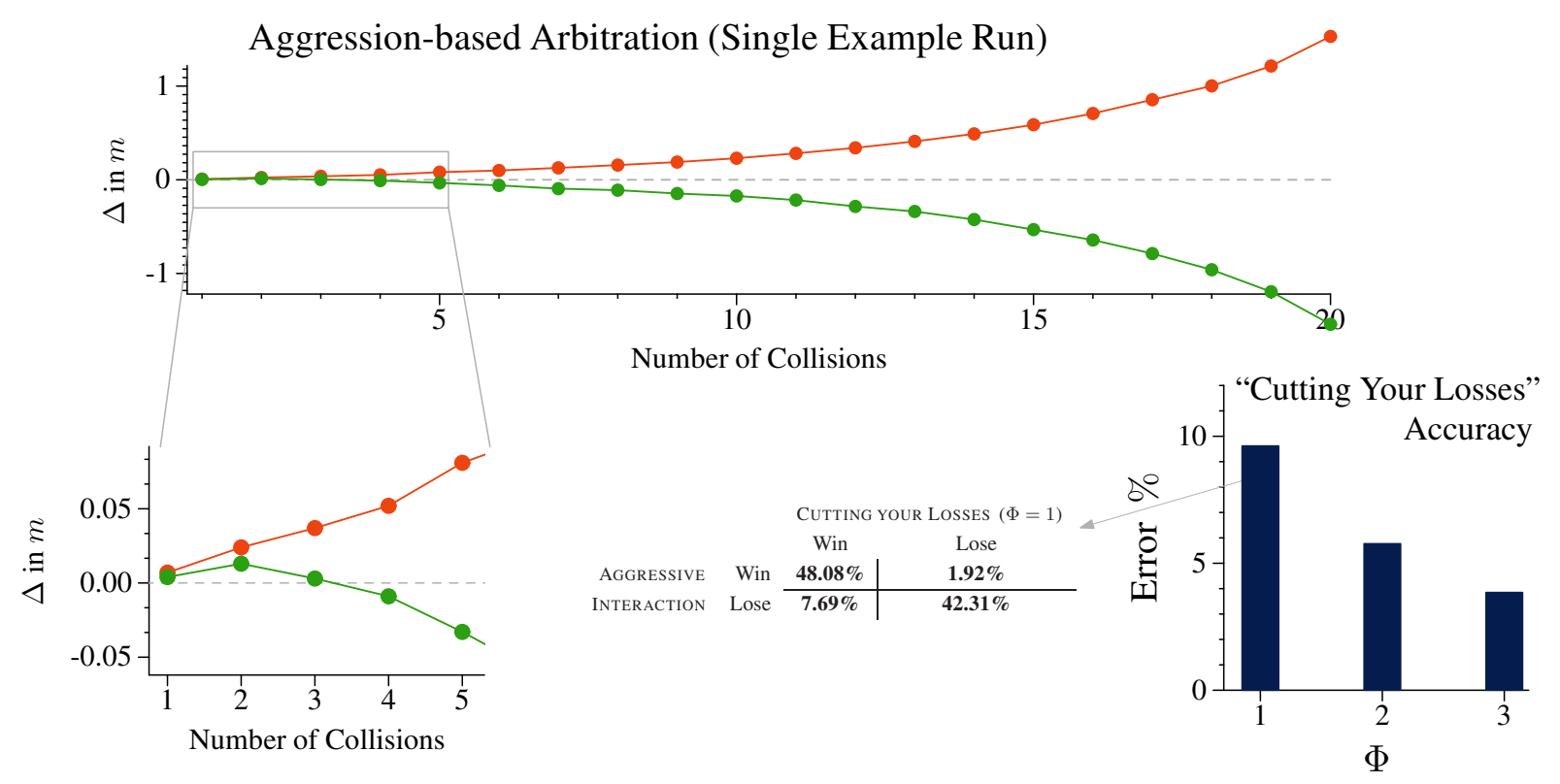

Fig. 21. The intuition behind the "cutting your losses" strategy is illustrated via an example of the aggression-based interaction. The sign of the single-time gain (denoted $\Delta$ in the graphs) indicates a likely win or loss. Waiting longer before measuring $\operatorname{sgn}(\Delta)$ reduces the estimate in the error due to the "escalation" dynamics.

\section{B. Cutting Your Losses}

In the earlier section we saw that the benefit from aggressive interactions is significantly reduced with the increase in cost. This happens for encounters with similar aggression levels. The usefulness of aggressive interactions can be improved by adding memory of recent performance. The robot measures and remembers the loss or gain in the shared space distance from the time it starts its aggressive display. If it repetitively loses distance, then it is unlikely to win the whole interaction. In such a situation it is beneficial to retreat. The greater the number of confirmations about the gain/loss in distance, the more accurate its decision.

Figure 21 shows a decrease in error with an increase in the number of confirmations. The trade off here is whether to take an early decision incurring less cost but 


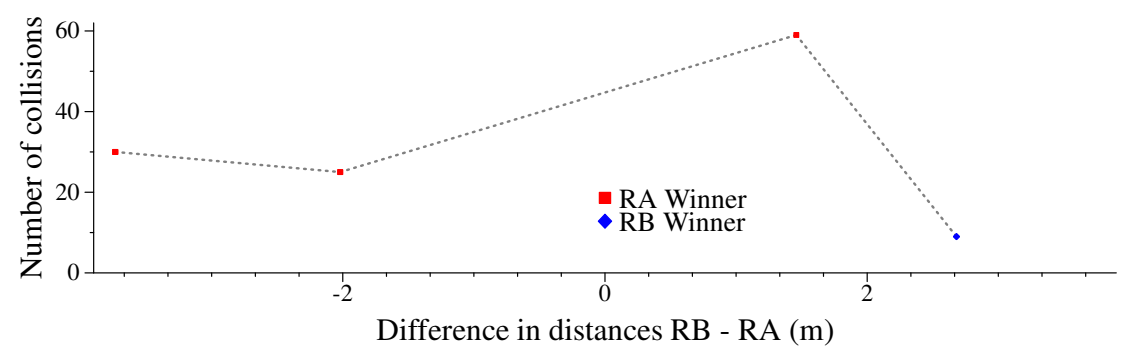

Fig. 22. Random walk - The large number of collisions illustrate the high cost involved. The horizontal-axis shows the difference in the local investment of $\mathrm{R}_{B}$ to that of $\mathrm{R}_{A}$ at the first instance of encounter in the girdle. The winner is determined on the basis of the backing distance drawn from a random distribution throughout the duration of interaction.

higher error percentages or whether to go for repeated confirmations incurring high cost but less error.

\section{Random Walk}

The attraction of random walk arbitration is its minimalism compared to other arbitration methods: robots do not need to sense or estimate their positions, since the position where the encounter takes place implicitly encodes the dynamic variable. Theoretical analysis is straight-forward providing an estimate of the likelihood that a robot will be given the right of way, given the initial position of encounter. Conducting sufficient physical robot experiments to observe this, however, is tedious. (Figure 22 illustrates four encounters as executed on our physical robots). We observe long arbitration duration when encounters occur at the center of the girdle: a phenomenon observed in all dynamic arbitration mechanisms. Yet this method is the most simple, has no explicit representation of aggression levels and still solves the purpose of arbitration without any physical damage to the agents. In terms of simplicity and informedness of arbitration methods random walk lies at one extreme 
of the spectrum. Later we also witness the other extreme when we introduce the Bayesian approach to resource arbitration in Chapter IX.

In the next chapter we take a closer look at the performance of dynamic and static arbitration methods with changing task structure. We selected rational aggression and linear dominance methods for comparison. 


\section{CHAPTER VI}

\section{INTERFERENCE — VARYING TASK RATIO AND GIRDLE LENGTH}

In order to compare the performance of dynamic and static arbitration methods across different task structure, we use our custom simulator. The simulator is designed to choose a particular task ratio, girdle length and an arbitration method. For dynamic arbitration, rational aggression method is chosen while linear dominance is the choice for static arbitration method. The custom simulator was run varying girdle lengths from 10 meters to 150 meters and, for each girdle length, task lengths covered by each robot was varied from 15 meters to 150 meters. The results represent complementary foci: either minimizing absolute arbitration cost via a static arbitration, or incurring whatever cost to ensure a dynamic arbitration.

\section{A. Dominance Model}

Figure 23 shows the performance in a girdle length of $30 \mathrm{~m}$ when $\mathrm{R}_{A}$ is the dominator.

Observations - Interesting regions from these plots were selected to investigate the interaction dynamics for the first 20,000 seconds (long enough to overcome initial transient and long-term behavior). These are marked with A-F in Figure 24.

1. A, B - Every time $\mathrm{R}_{B}$ makes an attempt to cross the girdle, it meets the dominator $\mathrm{R}_{A}$ and is pushed out of the girdle, making no progress whereas $\mathrm{R}_{A}$ finishes more than 15 laps during the alloted time. This is clearly a model of resource starvation. (Figure 24(a) and 24(b)).

2. E-In this case, $\mathrm{R}_{A}$ and $\mathrm{R}_{B}$ meet frequently and with $\mathrm{R}_{A}$ being the dominator, $\mathrm{R}_{B}$ is able to complete fewer trips than $\mathrm{R}_{A}$ with such frequent spatial interfer- 


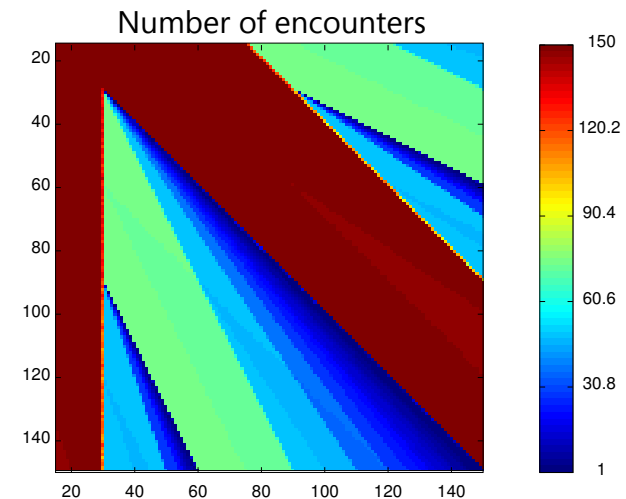

(a) Number of encounters

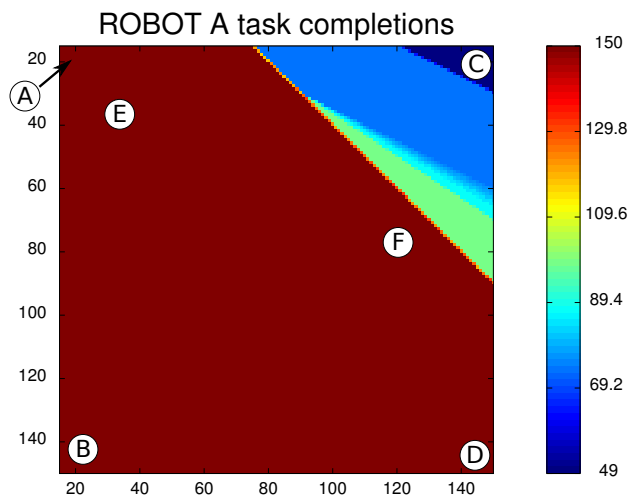

(b) $\mathrm{GL}=30$ Laps completed by $\mathrm{R}_{A}$

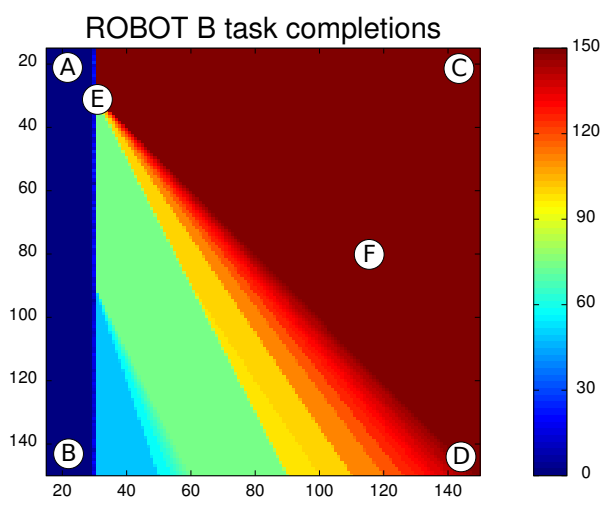

(c) $\mathrm{GL}=30$ Laps completed by $\mathrm{R}_{B}$

Fig. 23. Dominance for girdle length $=30 \mathrm{~m}, \mathrm{R}_{A}$ is dominator, horizontal-axis shows $\mathrm{R}_{A}$ 's task length, vertical-axis that of $\mathrm{R}_{B}$. (a)Color bars shows the relative number of encounters,(b),(c) Color bar shows the relative number of laps finished when at least one of the robots completes 150 laps. Points A to F are detailed in Figure 9. 


\section{$\Delta$ Girdle proportion of encounter}

RA laps completed

- - . RB laps completed

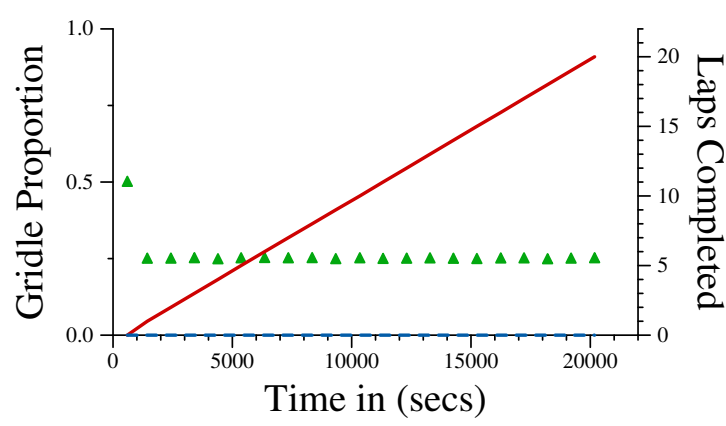

(a) $\mathrm{TA}=15 \mathrm{~TB}=15$

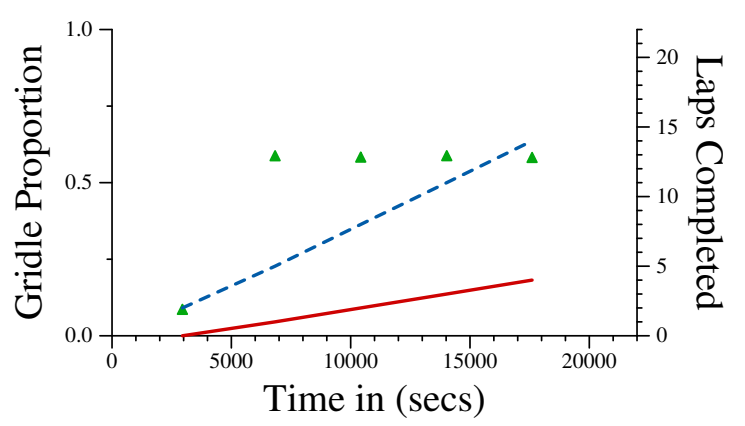

(c) $\mathrm{TA}=145 \mathrm{~TB}=20$

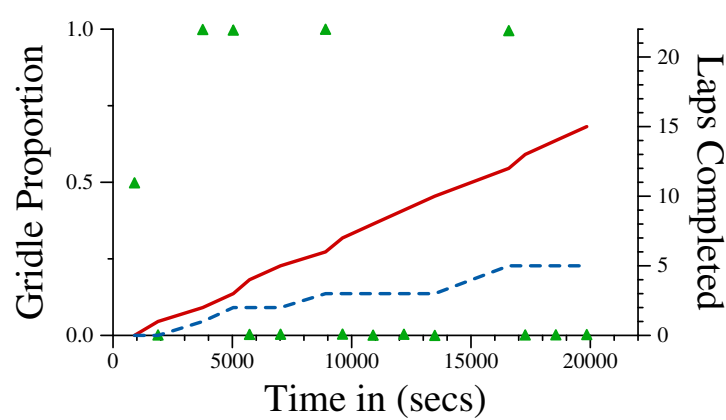

(e) $\mathrm{TA}=30 \mathrm{~TB}=30$

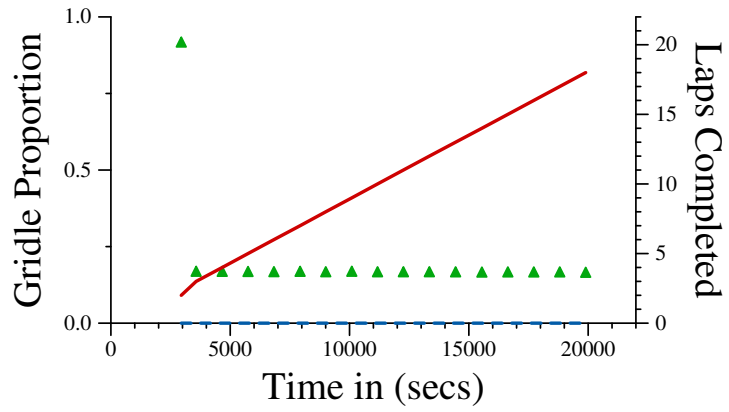

(b) $\mathrm{TA}=20 \mathrm{~TB}=145$

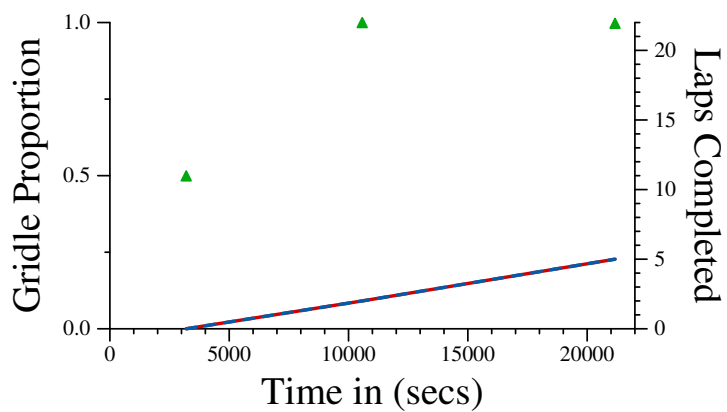

(d) $\mathrm{TA}=145 \mathrm{~TB}=145$

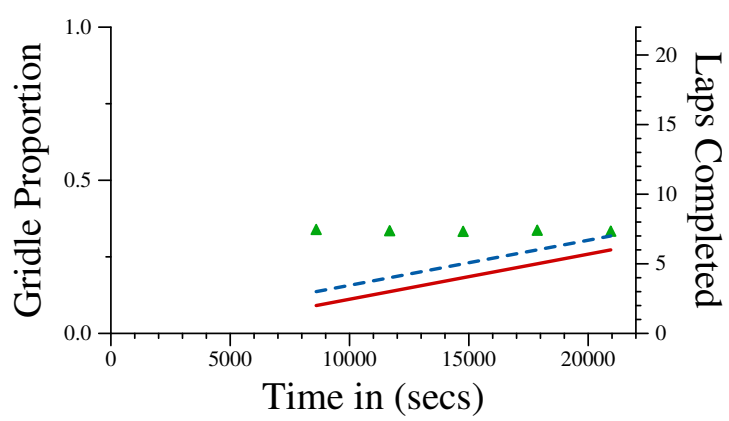

(f) $\mathrm{TA}=120 \mathrm{~TB}=80$

Fig. 24. Dominance for girdle length $=30 \mathrm{~m}, \mathrm{R}_{A}$ is dominator. The girdle proportions are with respect to the position of robot $R_{A}$. TA corresponds to the task length of $\mathrm{R}_{A}$, and $\mathrm{TB}$ to that of $\mathrm{R}_{B}$. 
ence.

3. $\mathrm{C}$ - The encounter position is close to $\mathrm{R}_{B}$ 's girdle entry point, so even if $\mathrm{R}_{B}$ retreats $\left(\mathrm{R}_{A}\right.$ being the dominator) the local investment made by $\mathrm{R}_{B}$ is less. $\mathrm{R}_{A}$ is the rational winner with aggression but at the cost of aggressive interaction time. Also they do not encounter one other every time $\mathrm{R}_{B}$ enters the girdle. But $\mathrm{R}_{B}$ 's shorter task allows it to complete more trips than $\mathrm{R}_{A}$. This explains the gap between the two lines in Figures 24(c).

4. F, D-The number of task iterations completed by both robots are almost equal. D belongs to the region where the number of encounters are less frequent (Figure 23(a)) and, if they occur at all, they are at the girdle end when $\mathrm{R}_{A}$ is about to exit (see Figure $24(\mathrm{~d})$ ). In such a situation $\mathrm{R}_{A}$ is the rational winner and the dominance hierarchy (with dominator $\mathrm{R}_{A}$ ) is the best model to follow.

From these results we see the variation in the task performance for different task ratios in a given girdle. Also task ratios can be grouped into sets and the behavior within a set is equivalent but there is significant difference in behavior as we move to a different set. This can be explained with an example. Consider the case where the task length of $R_{A}$ ranges from $20 \mathrm{~m}$ to $30 \mathrm{~m}$ but that of $R_{B}$ ranges from $20 \mathrm{~m}$ to $150 \mathrm{~m}$. The task performance of the robots for this entire set of task ratios is equivalent to what is shown in Figure 24(b) and is marked by the maroon vertical strip in Figure 23(a). The performance of each of the robots changes when the task lengths belong to a different set. The difference can be seen in the different colored regions of Figure 23. Another important conclusion that can be drawn from these results is that, there is no fixed best case arbitration mechanism to follow for every combination of task lengths. Going back to our previous example, where $R_{A}$ 's task length varied from $20 \mathrm{~m}$ to $30 \mathrm{~m}$ and $R_{B}$ from $20 \mathrm{~m}$ to $150 \mathrm{~m}$, we see resource starvation 
for $R_{B}$ as it is stopped in each of its attempts to cross the girdle and finish its task. Clearly in this scenario, dominance arbitration mechanism with $R_{A}$ being the dominator is detrimental to $R_{B}$ 's performance and for the cooperative system as a whole. Choosing $R_{B}$ as the dominator or following rational arbitration mechanism are better alternative arbitration mechanisms. The symmetrically opposite case occurs when $R_{A}$ 's task length ranges from $140 \mathrm{~m}$ to $150 \mathrm{~m}$ and $R_{B}$ 's from $20 \mathrm{~m}$ to $150 \mathrm{~m}$. $R_{A}$ being the dominator is a good arbitration mechanism to follow since either there are very few interferences or if there are frequent interferences, then they occur closer to $R_{B}$ 's girdle entry point. Even rational aggression method would have also resulted in $R_{A}$ being the winner but with an additional arbitration time.

\section{B. Aggression Model}

The aggression model of the simulator was run to do comparative study with the dominance model.

Collective best performance across varying girdle lengths: From Figure 25 it can be seen that for certain combinations of task lengths it takes significantly longer to complete 100 tasks traversals. One might think that this is due to frequent interferences for these task ratios. However, on plotting the interference count it was found that this is not always true. Thus the increased time duration is result of the increased time taken to resolve spatial interference occurring along these task ratios.

Below we discuss the scenario for a particular girdle length.

Collective vs. individual best performance for fixed girdle length: Consider the system performance when girdle length is 30m by comparing Figures 26(a) and 26(b). There are regions of high interference corresponding to regions of low task completion times. These are the instances where the robots met often but engaged in less costly 
aggressive interactions (cheap spots). On the other hand, there also exist regions of low interference but high task times. These regions correspond to high cost of aggressive interactions (high cost spots).

From Figure 26(c) and 26(d) we observe that in cheap spot regions each of $\mathrm{R}_{A}$ and $\mathrm{R}_{B}$ wins $50 \%$ of the encounters. We can conclude that these cheap spots are the regions of best collective performance, with a fair chance of right of way given to each robot. For the high cost regions it is beneficial to either follow the cheaper dominance arbitration mechanism or to add some wait time in the task lengths and shift the task ratios to the cheap regions.

Further investigation was done as to what happens for certain combinations of task lengths, similar to what was done for dominance model. Spots are marked by the letters in Figure 26(a) and positions of first encounter for every task iteration completed in the first 20,000 seconds are shown (Figure 27).

\section{Observations -}

1. $\mathrm{A}-\mathrm{R}_{A}$ and $\mathrm{R}_{B}$ meet frequently (Figure 26(b)). The position of encounter inside the girdle (Figure 27(a)) results in costly interactions, which is also illustrated by the time plots (Figure 26(a)). The number of task iterations completed by $R_{A}$ and $R_{B}$ is almost equal for the first 20,000 seconds of simulation and every time a rational winner is chosen. Figure 26(c) and 26(d) further show that both robots win an equal number of interactions and their wins have a particular dynamic structure: viz. they alternate in a zigzag pattern as Figure 27(a) illustrates. Compare this with how $\mathrm{R}_{B}$ performs when $\mathrm{R}_{A}$ is the dominator (Figure 24(a) and $24(\mathrm{~b})$ ). $\mathrm{R}_{B}$ did not make any progress during the time allotted. Here the trade-off is to, either to engage in costly aggressive interactions obtaining a rational winner, giving a fair chance of winning to each robot or to 
resort to (cheap) dominance and bias towards one agent.

2. $\mathrm{B}-\mathrm{R}_{A}$ and $\mathrm{R}_{B}$ do not meet as frequently here, but whenever they do, long aggressive interactions result: $\mathrm{R}_{B}$ is the rational winner in all cases. Compare this with Figure $24(\mathrm{~b})$ where $\mathrm{R}_{B}$ hardly made any progress. The best resolution mechanism would be with $\mathrm{R}_{B}$ as dominator so that no aggression cost needs be paid.

3. $\mathrm{C}$ - This is the reciprocal of case $\mathrm{B}$ and the earlier conclusion (but for $\mathrm{R}_{A}$ ) holds true.

4. $\mathrm{D}$ - The number of task iterations completed by $\mathrm{R}_{A}$ and $\mathrm{R}_{B}$ are almost equal. We notice that $\mathrm{D}$ belongs to the region where the number of encounters are less frequent (Figure 26(b)) and they occur at the ends of the girdle when $\mathrm{R}_{A}$ is about to exit (Figure 24(d)). We had earlier concluded that, in such a situation $\mathrm{R}_{A}$ is the rational winner and the dominance hierarchy (with $\mathrm{R}_{A}$ being the dominator) is the best interference model to follow. With these results one can conclude that aggressive arbitration is also a reasonable interference resolution mechanism. The reason being that these regions have cheap aggressive interactions.

5. E-Compare Figure 27(e) with Figure 24(e). The number of laps which $\mathrm{R}_{B}$ completes with aggressive signaling doubles compared with when it is dominated. However, the decrease in the number of laps of $\mathrm{R}_{A}$ is not that significant in both these modes of arbitration. One can observe frequent encounters in case of aggressive interactions, but all of these take place at the very ends of the girdle resulting in cheap arbitration, making it beneficial.

Another interesting observation from Figures 27(a) and 27(e) is that, there exists a limit cycle in terms of the position of encounter and with variation of the task ratio a new limit cycle emerges with a period almost double that of the earlier one. 
The observations outlined in this chapter addresses some of the major contributions of this thesis. Firstly the performance variation for different sets of task ratios signifies that if the agents can manipulate the task structure (example increase their task length by inducing a wait time while traversing the non-shared region), might be able to shift the system performance towards individual and collective good. Also, for certain combinations of task lengths we found rational arbitration to take long arbitration times whereas dominance arbitration gave the same result as rational arbitration but with no extra arbitration time. This clearly shows that an ideal arbitration mechanism can perform poorly due to high cost of arbitration. Another observation is that the most complex interference dynamics possible for a scenario is the limit cycle behavior. 


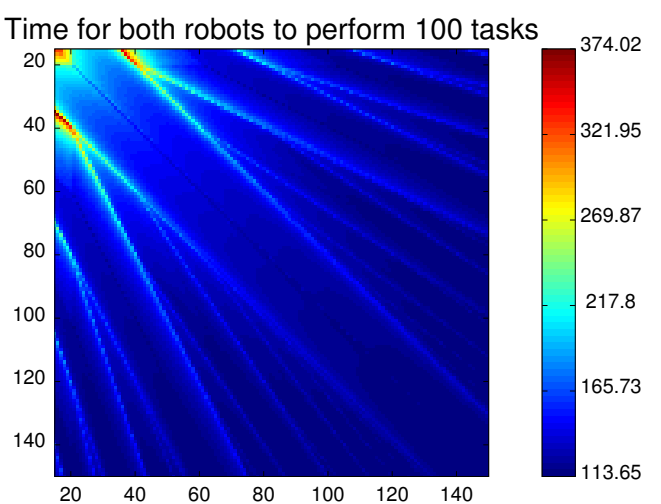

(a) Girdle Length $=20$

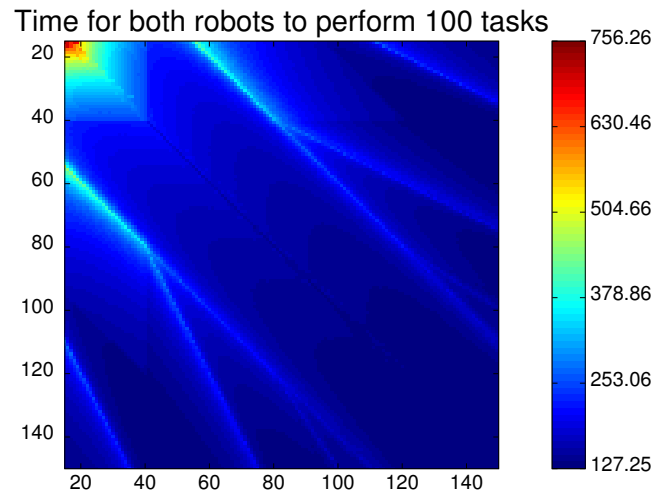

(c) Girdle Length $=40$

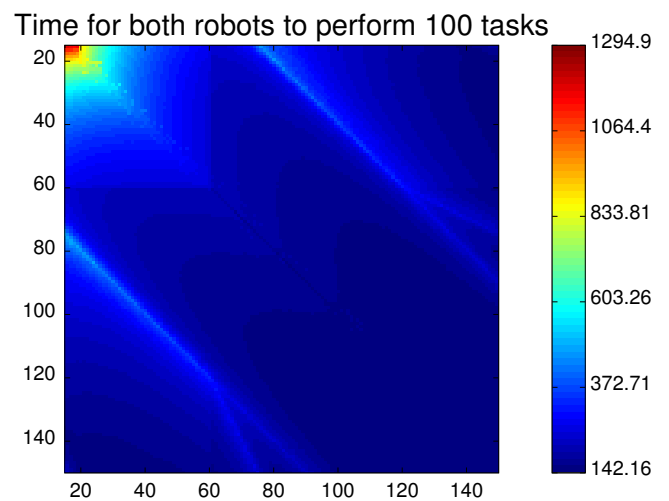

(e) Girdle Length $=60$

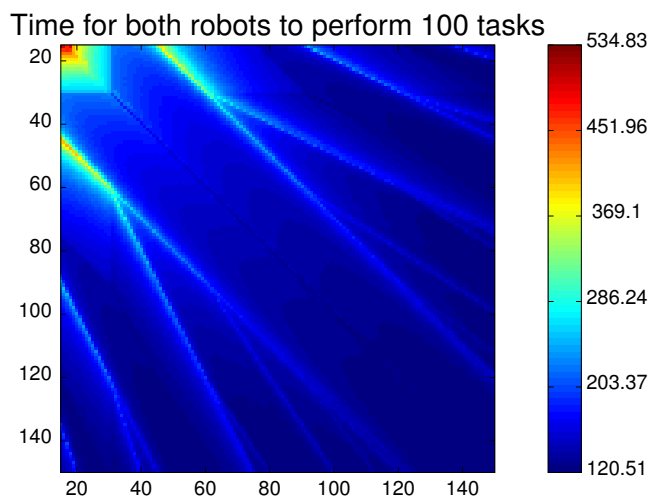

(b) Girdle Length $=30$

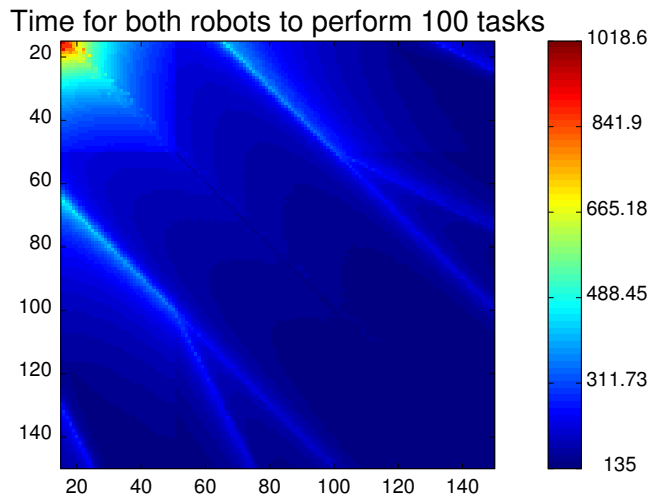

(d) Girdle Length $=50$

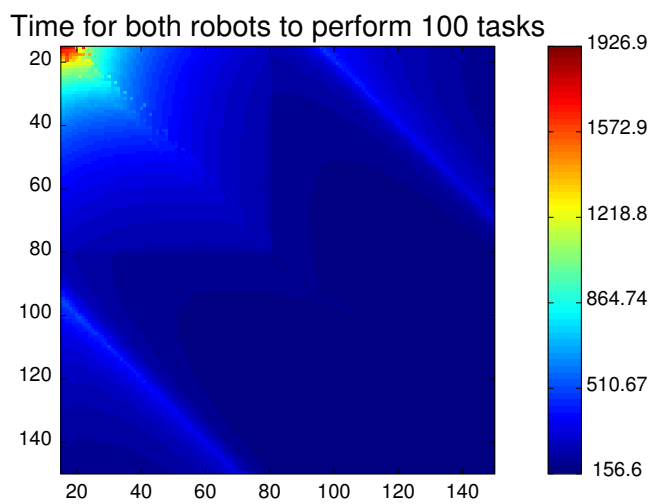

(f) Girdle Length $=80$

Fig. 25. Collective performance varying girdle length. The horizontal-axis shows $\mathrm{R}_{A}$ 's task length and vertical-axis that of $\mathrm{R}_{B}$. Color bars show the relative time to complete 100 navigation tasks in a row. 


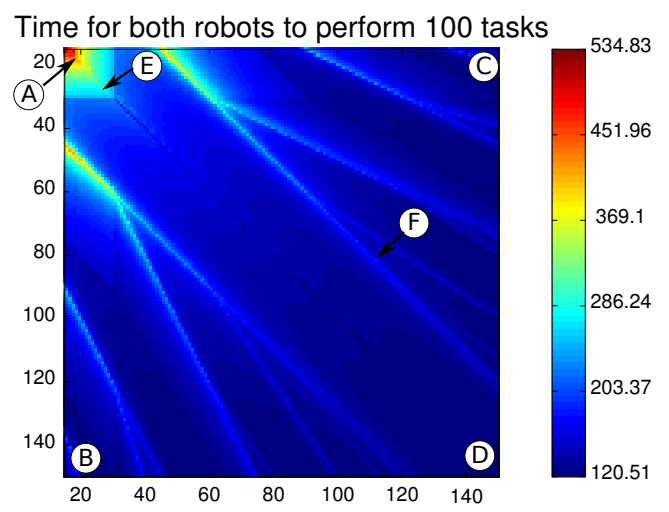

(a) $\mathrm{R}_{A} \mathrm{R}_{B}$ 's collective time plot

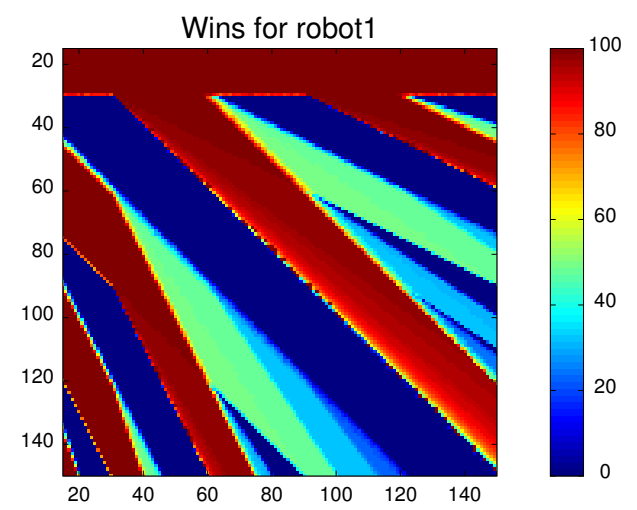

(c) $\mathrm{R}_{A}=30$ win count

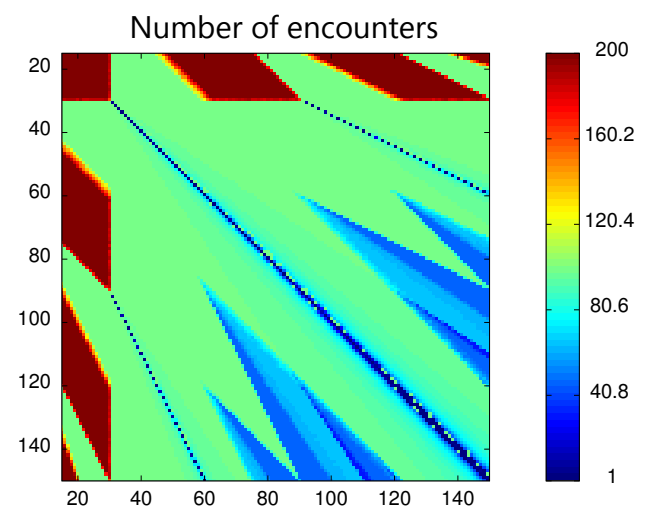

(b) Number of encounters

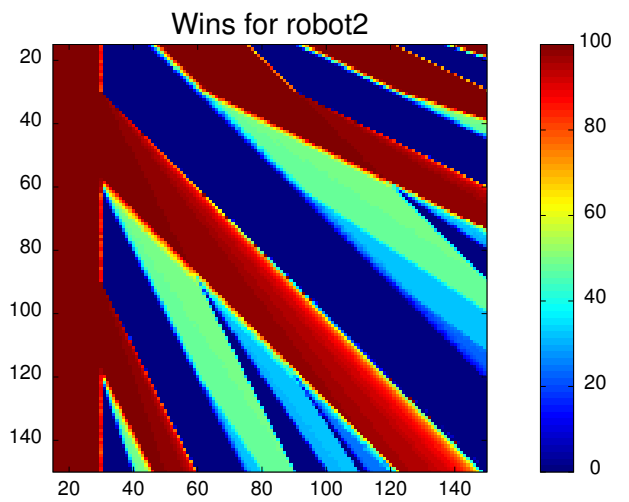

(d) $\mathrm{R}_{B}=30$ win count

Fig. 26. Collective vs. individual performance for girdle length $=30 \mathrm{~m}$. The horizontal-axis shows $\mathrm{R}_{A}$ 's task length and vertical-axis that of $\mathrm{R}_{B}$. Color bars show the relative measurements when 100 navigation tasks are completed in a row. 


\section{$\Delta$ Girdle proportion of encounter}

- RA laps completed

- - - RB laps completed

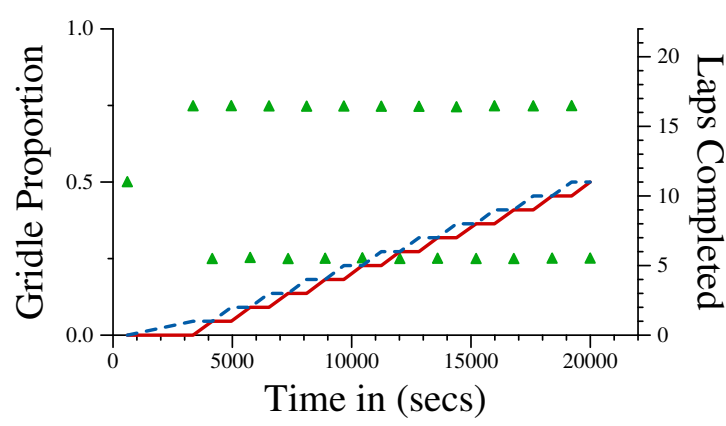

(a) $\mathrm{TA}=15 \mathrm{~TB}=15$

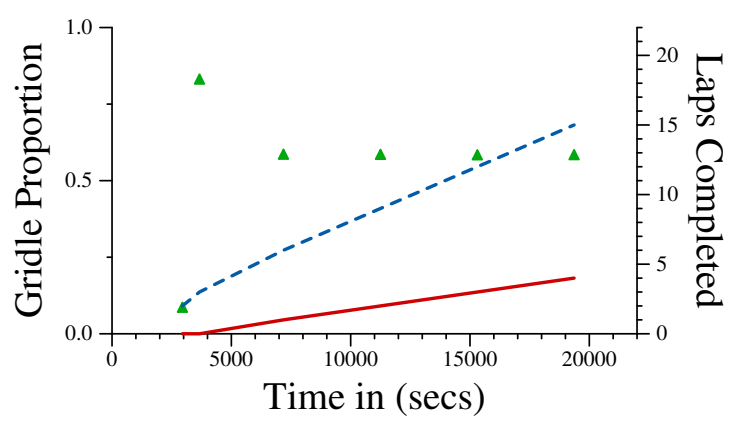

(c) $\mathrm{TA}=145 \mathrm{~TB}=20$

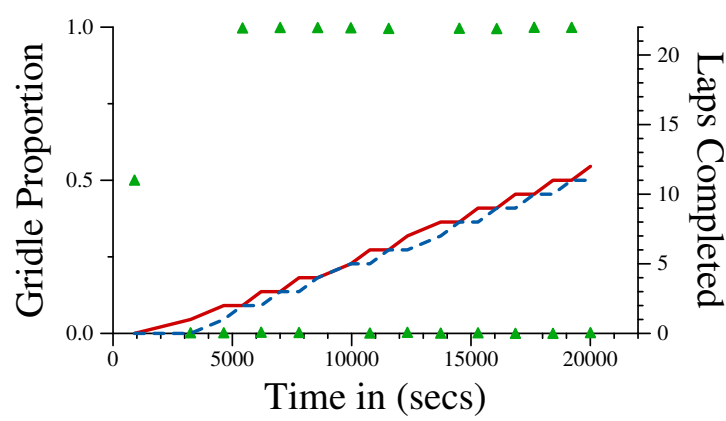

(e) $\mathrm{TA}=30 \mathrm{~TB}=30$

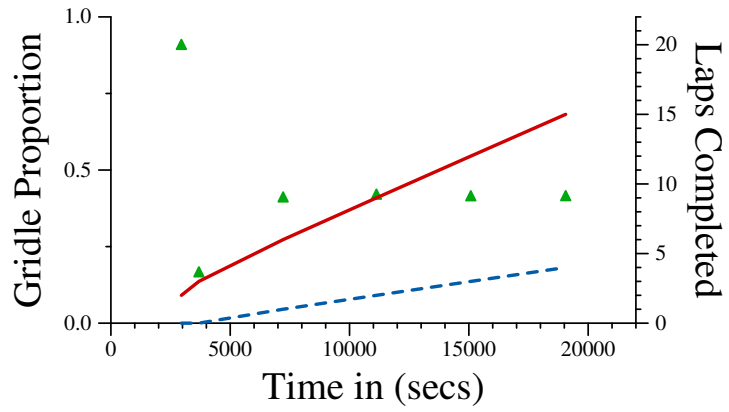

(b) $\mathrm{TA}=20 \mathrm{~TB}=145$

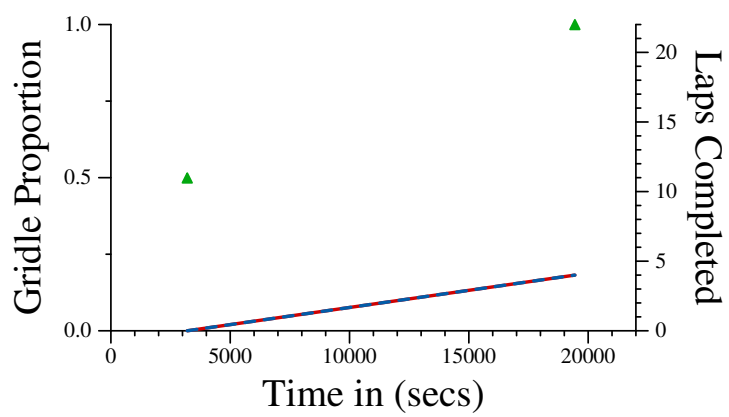

(d) $\mathrm{TA}=145 \mathrm{~TB}=145$

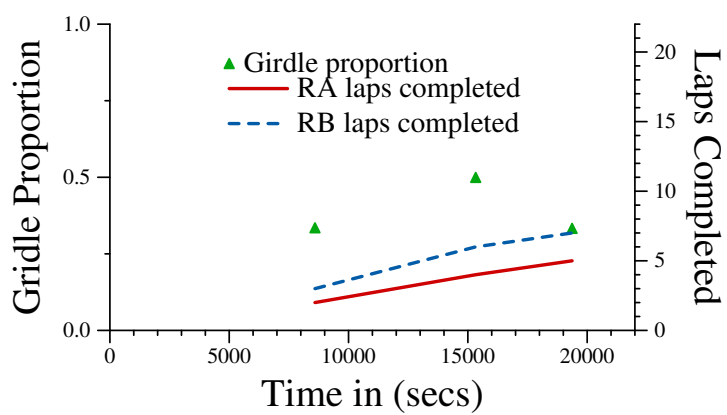

(f) $\mathrm{TA}=120 \mathrm{~TB}=80$

Fig. 27. Aggression GL30. The girdle proportion are with respect to the position of robot $\mathrm{R}_{A}$. 


\section{CHAPTER VII}

\section{AN ANALYSIS OF SHARED GIRDLE INTERFERENCE PROPERTIES}

This chapter addresses one of our main research questions of what environmental conditions causes spatial interference. It also answers the question of how many encounter free passes are needed to be checked before the next pass can be said to have spatial interference. The computational complexity for determining this is also formulated.

Definition 1. An idealized, noise-free interference scenario can be represented with a tuple $S=\left\langle G, T_{A}, T_{B}, I_{A}, I_{B}, m\right\rangle$. The first five elements $G, T_{A}, T_{B}, I_{A}, I_{B} \in \mathbb{R}^{+}$ are distances in some common unit: the girdle length, agent A's task, agent B's task, agent $A$ 's initial position $\left(I_{A} \leq T_{A}\right)$ and agent $B$ 's initial position $\left(I_{B} \leq T_{B}\right)$, respectively. The last element is the arbitration function $m: \mathbb{R}^{+} \times \mathbb{R}^{+} \times \mathbb{R}^{+} \rightarrow \mathbb{R}^{+} \times \mathbb{R}^{+}$.

The final element of the tuple can be written as $m\left(x_{i}\right) \mapsto p_{i}$ where $x_{i}$ is the position of encounter in a girdle normalized to have a unit length for an interference $i$. After the arbitration, the outcome of the arbitration $p_{i}$ is provided by $m$ and $0 \leq p_{i} \leq 1$ for a probabilistic model and $p_{i} \in\{0,1\}$ for deterministic model. As an example of arbitration in deterministic model, if $p_{i}=1$ and outcome is $R_{A}\left(p_{i}\right)$ then $R_{A}$ is the winner but if it is $R_{A}\left(1-p_{i}\right)$ then $R_{A}$ would have lost and $R_{B}$ is the winner with outcome $R_{B}\left(p_{i}\right)$. The resulting position of the agents is given by the function: $\operatorname{POS}\left(G, p_{i}\right) \mapsto\left(\operatorname{pos}_{A}^{\prime}, \operatorname{pos}_{B}^{\prime}\right)$. Here $G$ is the girdle length and $\operatorname{pos}_{A}^{\prime}$ and $\operatorname{pos}_{B}^{\prime}$ are the positions of the two agents after the arbitration. If the arbitration model does not use position information for arbitrating the shared resource as in the case of a static model, then it is denoted by $m()=.p_{i}$.

The homogeneous agents are assumed to travel with the same velocity $v$ both 
inside and outside the girdle. For interference scenario $S=\left\langle G, T_{A}, T_{B}, I_{A}, I_{B}, m\right\rangle$, agents begin at positions $I_{A}$ and $I_{B}$ in their own reference frame, which is considered to have origin at the girdle so that movement forward results in task performance. That is to say, the first $J_{A}=T_{A}-I_{A}$ units that agent $R_{A}$ proceeds forward involves executing the first task. Thereafter the next $G$ units denote space within the shared girdle, which may induce interference if agent $R_{B}$ attempts to proceed forward within the same space. The point at the end of the girdle (in the coordinate frame of each agent) is identified with the beginning of the agent's task. Analogously let $J_{B}=T_{B}-I_{B}$. Without loss of generality, assume $T_{B} \geq T_{A}$.

Definition 2. A 'cycle' is defined as the distance traveled from its position at $t=t_{0}$, to this same position, having moved through the girdle once.

Let $k$ denote the encounter-free cycle completions by $R_{A}$ and let $b$ denote the same for $R_{B}$. In other words, $k=T_{A}+G$ and $b=T_{B}+G$.

Definition 3. Agent $R_{B}$ is termed the clock, and the durations provided by cycles of $R_{B}$ are used to find the segment distances moved by the other agent $R_{A}$ during those regular time intervals.

Let robot $R_{B}$ start at time $t=0$, then it is evident that at time $t=\frac{1}{v} J_{B}$ it will be at a position just about to enter the girdle for the first time. Denote this time by $t_{0}$. Assuming that the robot $R_{B}$ does not encounter robot $R_{A}$ in the girdle, it will next be at this same threshold of the girdle at time $t_{1}=t_{0}+\frac{1}{v}\left(G+T_{B}\right)$. Let $\Delta t=\frac{1}{v}\left(G+T_{B}\right)=t_{1}-t_{0}$

Now suppose that robots $R_{A}$ and $R_{B}$ encounter each other in the girdle at some time. Consider the first such time: $t_{c}$. If robot $R_{B}$ has performed $n$ complete task rotations, then $t_{0}+n \Delta t \leq t_{c} \leq t_{0}+n \Delta t+\frac{1}{v} G$. (Here $n$ is a natural number, or 
possibly zero.) Let $m$ denote the number of cycles that robot $R_{A}$ has completed until time $t=t_{c}$. Note that from the assumption on task lengths, above, $n \leq m$.

Definition 4. Let $I_{A}$ and $I_{B}$ be the initial positions of $R_{A}$ and $R_{B}$, then a necessary condition for them to encounter even before completing their first cycle is

$$
\left|J_{A}-J_{B}\right| \leq G \text { for } n=0 \text { and } m=0 \text {. }
$$

It can be easily observed that if $J_{A} \leq J_{B}$, and if the above condition is true, then by the time $R_{B}$ starts entering the girdle, $R_{A}$ is already traversing through the girdle in the direction opposite to that of $R_{B}$. Thus, both would face each other inside the shared space resulting in spatial interference. By symmetry, the converse is also true when $J_{B} \leq J_{A}$.

Lemma 1. If at time $t$ robot $R_{B}$ is at a position such that it is just about to enter the girdle, then a necessary and sufficient condition for both robots to encounter one another in next passing through the girdle, is for robot $R_{A}$ to be positioned at a distance which is at most $2 G$ units away from $R_{B}$ 's position at time $t$.

Proof. While navigating through the environment if at any point in time $t, R_{B}$ is in a position where it is just about to enter the $G$ long common traversal region and if $R_{A}$ is positioned at a distance which is less than $G$ from $R_{B}$ 's position at time $t$, then $R_{A}$ would enter the girdle before $R_{B}$ exits, causing interference. Thus interference occurs when both are $2 G$ distance apart at a point in time when one of them is about to enter the $G$ long girdle.

Bézout's lemma Let $a, b \in \mathbb{Z}$ such that $a$ and $b$ are not both zero. Let $\operatorname{gcd}(a, b)$ be the greatest common divisor of $a$ and $b$. Then:

$$
\exists x, y \in \mathbb{Z}: a x+b y=\operatorname{gcd}(a, b) .
$$


Theorem 2. Given the task lengths $T_{A}$ and $T_{B}$ for robots $R_{A}$ and $R_{B}$ respectively and the girdle length $G$, if $g=\operatorname{gcd}(b, k)$, then an encounter takes place when either of the following conditions is true:

1. If $g<2 G$,

2. If $g \geq 2 G$ and $r^{\prime} g+a \leq\left(r^{\prime}+1\right) g \leq r^{\prime} g+a+g$, where $r^{\prime}=\left\lfloor\frac{T_{A}-G-a}{g}\right\rfloor$.

Again consider robot $R_{B}$ as the clock providing us with the timings for robot $R_{A}$ 's movement. Let robot $R_{B}$ start at time $t=0$ and robot $R_{A}$ start from an initial position $I_{A}$. Consider a scenario when both travel encounter-free until $R_{B}$ finishes $i$ cycles of length $T_{B}+G$. Robot $R_{A}$ 's traversal with respect to $R_{B}$ as the clock can be listed as follows:

1. At time $t_{0}=\frac{J_{B}}{v}, R_{A}$ is at distance

$$
I_{A}+J_{B}
$$

2. At time $t_{1}=t_{0}+\frac{T_{B}+G}{v}, R_{A}$ is at distance

$$
I_{A}+J_{B}+G+T_{B}
$$

3. At time $t_{i}=t_{0}+i\left(\frac{T_{B}+G}{v}\right), R_{A}$ is at distance

$$
I_{A}+J_{B}+i\left(G+T_{B}\right)
$$

Let $I_{A}+J_{B}=a, G+T_{B}=b$ and $T_{A}+G=k$. Robot $R_{A}$ covers distance $a+i . b$ by moving in cycles of length $k$.

When $R_{B}$ finishes $i$ cycles, let $R_{A}$ be about to complete its $j^{\text {th }}$ cycle of length $k=T_{A}+G$. Robots $R_{A}$ and $R_{B}$ will meet if at $j^{\text {th }}$ cycle of $R_{A}$ and $i^{\text {th }}$ cycle of $R_{B}$ both are inside the girdle. Given only the lengths of the individual tasks and girdle, 
one can determine if such an interference scenario exists or not. This is a two-step process.

1. First, one needs to determine if there exists any linear combination of the cycle counts $i$ and $j$ of $R_{A}$ and $R_{B}$, such that after doing their respective $i^{\text {th }}$ and $j^{\text {th }}$ cycles they come to the same position from where they had started.

2. Next one also needs to determine if this same time same place SPST position falls within a common traversal region.

The former can be determined using Bézout's lemma and the later follows from Lemma 1.

- Step 1: $R_{A}$ and $R_{B}$ will be at the same place after traversing for the same lengths of time, but each covering a different cycle length only if there exists a greatest common divisor of their cycle lengths. This is termed as $g=\operatorname{gcd}(b, k)$, where $b=T_{B}+G$ and $k=T_{A}+G$. Existence of the greatest common divisor ensures that any linear combination of the cycle counts, $i$ and $j$ of $R_{A}$ and $R_{B}$ respectively, can be covered in steps of length $g$. If they meet they will meet after $R_{A}$ covers distance:

$$
a+i \cdot b-j \cdot k, \forall i, j
$$

Applying Bézout's lemma the above expression becomes:

$$
\Rightarrow a+r \cdot g \quad \text {, where } r=0,1 \ldots\left(\frac{b}{g}-1\right) \text { and } g=\operatorname{gcd}(b, k) \text {. }
$$

Once the existence of ' $g$ ' is determined the next thing is to find out if after traversing the above distance the next ' $g$ ' steps makes them meet each other inside the girdle causing interference. 
- Step 2: There can be two distinct cases for the next ' $g$ ' step to fall within the common traversal region. In each of these cases $R_{B}$ is considered to be the clock and $R_{A}$ is moving with respect to the clock.

1. $g<2 G$ - If this condition is true then after every $g$ steps that $R_{A}$ takes there will be encounter, since each of these steps will fall inside the $2 G$ interference region.

2. If $g \geq 2 G-$ In this case, there is a chance that $R_{A}$ takes its next $g$ steps from a position which makes it fall inside the $2 G$ interference region, resulting in an encounter. Let $R_{A}$ travel in step sizes of $g$ for $r^{\prime}$ times before it first passes through the girdle. So far it was traveling in the non-shared region and there is no chance of encounter. The length of the non-shared region is $T_{A}-G-a$. Hence,

$$
r^{\prime}=\left\lfloor\frac{T_{A}-G-a}{g}\right\rfloor .
$$

Now what can happen when $R_{A}$ takes the $r^{\prime}+1^{\text {th }}$ step of size $g$ ? If this step falls inside the $2 G$ interference region then there will certainly be an encounter. In other words, the $r^{\prime}+1^{\text {th }}$ step needs to falls within $T_{A}-G$ and $T_{A}+G$ for interference to happen. Thus the condition for occurrence of encounter is the following:-

$$
T_{A}-G \leq\left(r^{\prime}+1\right) g \leq T_{A}+G
$$

A maximum of $r^{\prime}=\left\lfloor\frac{T_{A}-G-a}{g}\right\rfloor$ passes of $R_{A}$ through the girdle needs to be checked to find out if $r^{\prime}$ encounter free iterations have taken place. Thereafter one may determine whether the next $\left(r^{\prime}+1\right)^{t h}$ pass will result in an interference or not. If the cycle lengths $k$ and $b$ of $R_{A}$ and $R_{B}$ 
respectively are co-prime, then $\operatorname{gcd}(b . k)=1$. Hence $r^{\prime}=T_{A}-G$. Thus a relatively large number of checks of encounter free passes needs to be done to determine when the next encounter will take place.

Thus, given any girdle and task lengths, and the initial starting positions of two robots, it is possible to determine how many encounter-free attempts are needed to find out if there will be an interference in the next pass through the girdle. An obvious question is how fast can this be determined? The gcd of two numbers can be found out by Euclid's algorithm which in the worse case $O(h)$ divisions where $h$ is the number of digits in the smallest of the two numbers. Thereafter the calculation of $r^{\prime}$ takes constant time with a single division operation, followed by $r^{\prime}$ checks of encounter free iterations. Thus the entire computation is linear with either $h$ or $r^{\prime}$ dominating the worse case time, depending on whichever is larger.

Definition 5. If the conditions of Lemma 1 and 2 are satisfied, and the robots meet then the state of the system after the resolution of interference by the function $m\left(x_{i}\right) \mapsto p_{i}$ can be modelled as POS $\left(g, p_{i}\right) \rightarrow\left(\operatorname{pos}_{A}^{\prime}, \operatorname{pos}_{B}^{\prime}\right)$

With our present interference model, two cases follow:

1. Aggressive interference - In an aggressive encounter robot $R_{A}$ will win the fight with a probability dependent on the position of encounter $x$, where $x$ is the position inside a normalized girdle length. Considering a noise free interference scenario the state of the system can be modeled after the aggressive encounter as follows:

$$
m(x)=p_{i}, \operatorname{POS}\left(g, p_{i}\right)= \begin{cases}\left(0, T_{B}\right) & \text { if } x>0.5 \\ \left(T_{A}, 0\right) & \text { if } x<0.5\end{cases}
$$

where probability of $R_{A}$ being the winner is: 


$$
\begin{aligned}
& \operatorname{Pr}\left(\text { Winner }=\text { Robot }_{1} \mid \text { Girdle_Pos }_{-}=y\right) \\
& \quad= \begin{cases}1 & \text { if } x<0.5 \\
0 & \text { if } x>0.5\end{cases}
\end{aligned}
$$

However in a noise free scenario the outcome of the arbitration cannot be determined for center position, i.e. when $x=0.5$. We can refer to our empirically determined noise (Chapter IV) to define the winner when $x \approx 0.5$ :

$$
\begin{gathered}
\operatorname{Pr}\left(\text { Winner }=\text { Robot }_{1} \mid \text { Girdle_Pos }=y\right) \\
= \begin{cases}1 & \text { if } x \leq 0.4725 \\
\frac{0.54750-x}{0.075} & \text { if } x \leq 0.54750, \\
0 & \text { if } x>0.54750 .\end{cases}
\end{gathered}
$$

2. Dominance interference - In a dominance hierarchy model, the outcome is deterministic and the winner is always the dominant robot. The state of the system after dominance interference can be modeled as follows:

$$
m(.)=p_{i}, \operatorname{POS}\left(g, p_{i}\right)= \begin{cases}\left(0, T_{B}\right) & \text { if } \mathrm{A} \text { is dominant } \\ \left(T_{A}, 0\right) & \text { otherwise }\end{cases}
$$

The state of the system will be the same in both a noise free scenario and when noise is present. 


\section{CHAPTER VIII}

\section{DECISION TREE ENCODING OF ARBITRATION MODELS}

\section{A. Definitions}

Interference Switch - Given the tuple $\left\langle G, T_{A}, T_{B}, I_{A}, I_{B}, m\right\rangle$ as input, Theorem 2 can answer the question of whether there will be an interference when two robots attempt to pass through the shared girdle space. Whenever the tuple input changes this question needs to be re-evaluated. Such evaluation points during the course of an interaction can be represented as switches where 'ON' denotes that an encounter will take place while 'OFF' is an indication of no encounter.

Interference Point — An encounter takes place when an interference switch is turned 'ON'. This is termed as the interference point and the outcome from an interference is the decision as to which agent should get access to the resource. This decision is based on the arbitration mechanism used to arbitrate the shared resource. Any particular interference point visited during a course of interaction depends on two factors - (a).How the preceding interference switches are set and, (b). If it is not the very first interference then it also depends on how the preceding interference was resolved.

Termination Path - An interaction sequence can follow a path in the decision model which eventually leads to a 'Never Meet' state. Once this state is reached it guarantees that there will be no future interferences when an agent makes a pass through the shared region. Such a path is termed as a termination path.

Non Termination Path - An interaction sequence can follow a path in the decision model which does not take it to the 'Never Meet' states. If the decision path misses all the 'Never Meet' states then all future traversals will loop forever along the 
paths of the interference points. Such a path is termed as a non-termination path.

String of interference pattern - Every distinct interference point can be labeled with a particular character. A sequence of characters during the course of task traversal represents the sequence of interference points visited. Such a string of characters represents an interference pattern.

These can be broadly divided into two categories depending on whether they are finite or not.

- Terminating strings - The string of interference pattern which results from a terminating path has finite length and are termed as terminating strings. A terminating path which repeatedly visits an interference point $i$ will be represented by the terminating string ' $\cdots i^{*}$.

- Non-terminating strings - The ones which follow the non-terminating path keeps visiting interference points forever and these are termed as non-terminating strings. These are represented by assigning the $\infty$ symbol to all the character/characters corresponding to the interference point/points visited infinitely. Example of such a sequence is $\alpha(\beta)^{\infty}$ where interference point represented by character $\beta$ will be visited infinitely for all future interactions.

The following theorem results from the analysis of the shared girdle interference properties and the generated patterns of interference of any interaction sequence.

Theorem 3. The corresponding decision tree model of all arbitration models (mentioned so far) for any given girdle and task ratio can have a maximum of three unique interference points. All possible interference patterns are generated by visiting just these three interference points.

Proof. The theorem can be proved using the simple fact that the number of possible unique interference points depends on the number of possible ways the preceding 
interference switch can be turned ON. This in turn depends on the number of possible unique inputs to the interference switch. An input to an interference switch is defined by the tuple $\left\langle G, T_{A}, T_{B}, I_{A}, I_{B}, m\right\rangle$. First we need to determine the number of variables in this tuple in order to find all possible tuple combinations. For any given girdle length and task ratio $G, T_{A}, T_{B}, m$ are constant for an arbitration model. The only variables are the initial conditions $I_{A}, I_{B}$. These have a value at the very start of the robot traversal task. This forms the input to the first interference switch. Since the robots can start anywhere in the non-shared region, we have $0 \leq I_{A} \leq T_{A}-G$ and $0 \leq I_{B} \leq T_{B}-G$ for the very first traversal. Thereafter for subsequent traversals, $I_{A}, I_{B}$ needs to be recalculated every time they are positioned at the origin. For an arbitration model, the only positioning which are of interest are those which results from an encounter. If the first interference point was turned ' $\mathrm{ON}$ ', then the following interference can have two outcomes:-

1. $R_{A}$ wins and finishes the traversal through the shared space and is ready to begin the next task traversal. Thus, $I_{A}=0 . R_{B}$ loses and is pushed at the other end of the girdle and is yet to finish traversing through the girdle. Its position at the girdle origin is reset to $T_{B}-G$. At this instance the question of whether a future interference is possible needs to be re-evaluated. Theorem 2 takes the new tuple $\left\langle G, T_{A}, T_{B}, 0, T_{B}-G, m\right\rangle$ as the input to evaluate this question and this represents the second unique interference point.

2. $R_{B}$ wins and the result is symmetrical to $R_{A}$ 's win. This forms the third unique interference point.

Hence any arbitration model for a sequence of interactions can have three interference points. If each of these points is represented with a character, then a sequence 
of interferences in any arbitration model can be represented as a combination of just these three characters. The most important result which follows from this is that, given any string of interference pattern one can determine the exact sequence of interferences in the interaction and also the outcome of these encounters for any given arbitration model. An arbitration model can also produce strings which are unique to the model. If such strings are reported one can even determine the arbitration mechanism used by the agents to resolve an encounter.

The interference switches along with the resulting interference points can be listed as follows:-

1. $(\mathrm{S} 0, \alpha)-\mathrm{S} 0$ is always the first interference switch that any interaction sequence has to go through. S0 takes as input the tuple $\left\langle G, T_{A}, T_{B}, I_{A}, I_{B}, m\right\rangle$, where $I_{A}$ and $I_{B}$ denote the initial positions of $R_{A}$ and $R_{B}$ even before they have started their respective traversal tasks. If S0 is turned ' $\mathrm{ON}$ ' then the subsequent encounter will be the very first encounter in the entire interaction sequence. This interference point is denoted by $\alpha$.

2. $(\mathrm{S} 1, \beta)$ - If $\mathrm{S} 0$ is turned 'ON', then the robots will meet for the first time when both simultaneously attempt to pass through the shared girdle. If this encounter results in $R_{A}$ 's win, then the resulting tuple $\left\langle G, T_{A}, T_{B}, 0, T_{B}-G, m\right\rangle$ is a unique pre-condition where the question of a possible future encounter needs to be reevaluated. Hence, this is fed as the input to the next interference switch S1 to answer the question of whether there will be any more future encounters. If S1 is ' $\mathrm{ON}$ ' then the subsequent interference forms the next interference point $\beta$.

3. $(\mathrm{S} 2, \gamma)-\mathrm{S} 2$ behaves symmetrical to that of $\mathrm{S} 1$. If $\mathrm{S} 0$ is turned 'ON' and on encounter $R_{B}$ wins, then the resulting tuple $\left\langle G, T_{A}, T_{B}, T_{A}-G, 0, m\right\rangle$ is fed as input to the interference switch S2 to answer the question of future encounters. 


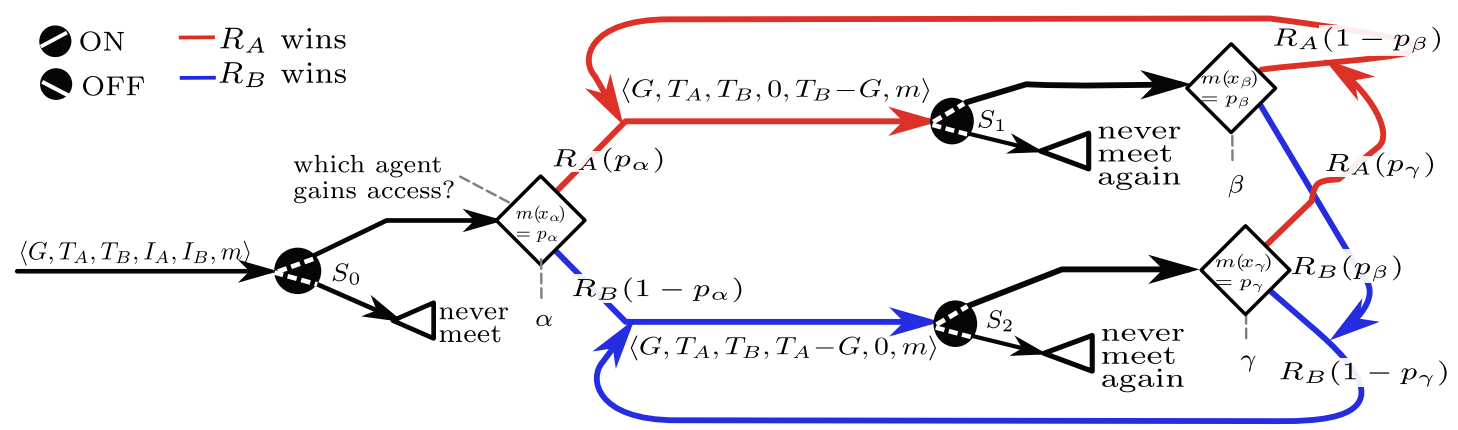

Fig. 28. Decision tree encoding when the arbitration is dynamic deterministic or dynamic probabilistic.

B. Decision Tree Encodings of Arbitration Models

The taxonomy of arbitration models identifies four categories of arbitration models and each of these can be represented with a corresponding decision tree model:-

1. Dynamic deterministic - A dynamic deterministic model utilizes the information of an encounter to arbitrate the shared resource.

Given a particular interference scenario there is a definitive answer as to who should be awarded the resource. Thus $p_{\alpha}, p_{\beta}, p_{\gamma} \in\{0,1\}$. Figure 28 shows the decision paths of such an arbitration. Since dynamic arbitration utilizes encounter information to arbitrate the shared resource there is one interference point corresponding to each interference switch. Which interference point will be visited depends on how the interference switches are set and how a preceding interference is arbitrated (provided it is not the first interference).

Table II shows the string of interference patterns generated for all possible valid combinations of switches and arbitration results. The values of $p_{\alpha}, p_{\beta}, p_{\gamma}$ govern the arbitration results. This forms the complete set of interference patterns that a dynamic deterministic model could possibly generate. Both terminating and non-terminating strings are generated by this model. 
Table II. Strings generated from a dynamic deterministic model

\begin{tabular}{|c|c|c|c|c|c|c|}
\hline $\mathbf{S}_{0}$ & $\mathbf{p}_{\alpha}$ & $\mathbf{S}_{1}$ & $\mathbf{p}_{\beta}$ & $\mathbf{S}_{2}$ & $\mathbf{p}_{\gamma}$ & Strings \\
\hline SOFF & - & - & - & - & - & $\lambda$ \\
\hline$\Theta O N$ & 1 & SOFF & - & - & - & $\alpha$ \\
\hline $\boldsymbol{\theta O N}$ & 1 & $8 \mathrm{ON}$ & 1 & ८OFF & - & $\alpha \beta$ \\
\hline$\Theta O N$ & 1 & $\Theta O N$ & 0 & - & - & $\alpha(\beta)^{\infty}$ \\
\hline ?ON & 1 & $=O N$ & 1 & $=\mathrm{ON}$ & 0 & $\alpha \beta(\gamma)^{\infty}$ \\
\hline$\approx \mathrm{ON}$ & 1 & $\Theta O N$ & 1 & $\Theta \mathrm{ON}$ & 1 & $\alpha(\beta \gamma)^{\infty}$ \\
\hline$\Theta O N$ & 0 & - & - & SOFF & - & $\alpha$ \\
\hline$\Theta O N$ & 0 & SOFF & - & $\theta \mathrm{ON}$ & 1 & $\alpha \gamma$ \\
\hline 气ON & 0 & - & - & $\Theta O N$ & 0 & $\alpha(\gamma)^{\infty}$ \\
\hline$\theta \mathrm{ON}$ & 0 & $=O N$ & 0 & $\theta \mathrm{ON}$ & 1 & $\alpha \gamma(\beta)^{\infty}$ \\
\hline$\theta \mathrm{ON}$ & 0 & $=\mathrm{ON}$ & 1 & $=\mathrm{ON}$ & 1 & $\alpha(\gamma \beta)^{\infty}$ \\
\hline
\end{tabular}


There are four possible termination paths generating terminating strings and the remaining six non-termination paths generate the infinite patterns. There is just one possible way by which interaction proceeds with no encounter and this is represented with an empty string. Thus given any string of interference pattern we can say how the interference took place, both in terms of the sequence of interference points visited and the outcome of the encounter at this points.

2. Dynamic probabilistic - A dynamic probabilistic model utilizes the information of an encounter to decide its outcome. Also, given a particular interference scenario there is a chance for an agent to win the encounter. Hence, $0 \leq$ $p_{\alpha}, p_{\beta}, p_{\gamma} \leq 1$. Figure 28 shows the possible decision paths taken by a dynamic probabilistic arbitration model. The difference between the probabilistic and static models of dynamic arbitration is in the probabilities with which a robot can gain access to a shared resource. This in turn determines the resulting patterns of interference. The strings in Table III show the possible patterns of interference in a dynamic probabilistic model. These are no longer unique as is the case with the earlier model, but they belong to a set of strings and there can be just four such sets.

3. Static deterministic - Figure 29, shows the decision tree encoding of a static deterministic arbitration model. As per the definition, static arbitration uses no information from the current encounter to resolve a resource conflict. The winner is pre-determined since deterministic arbitration always awards the resource to the same agent, given the same interference scenario. The interference points of the corresponding decision model does not use any information from the encounter to arbitrate the shared resource. All three interference points have the same characteristics. Hence in Figure 29 we have $\alpha=\beta=\gamma=\rho$. 


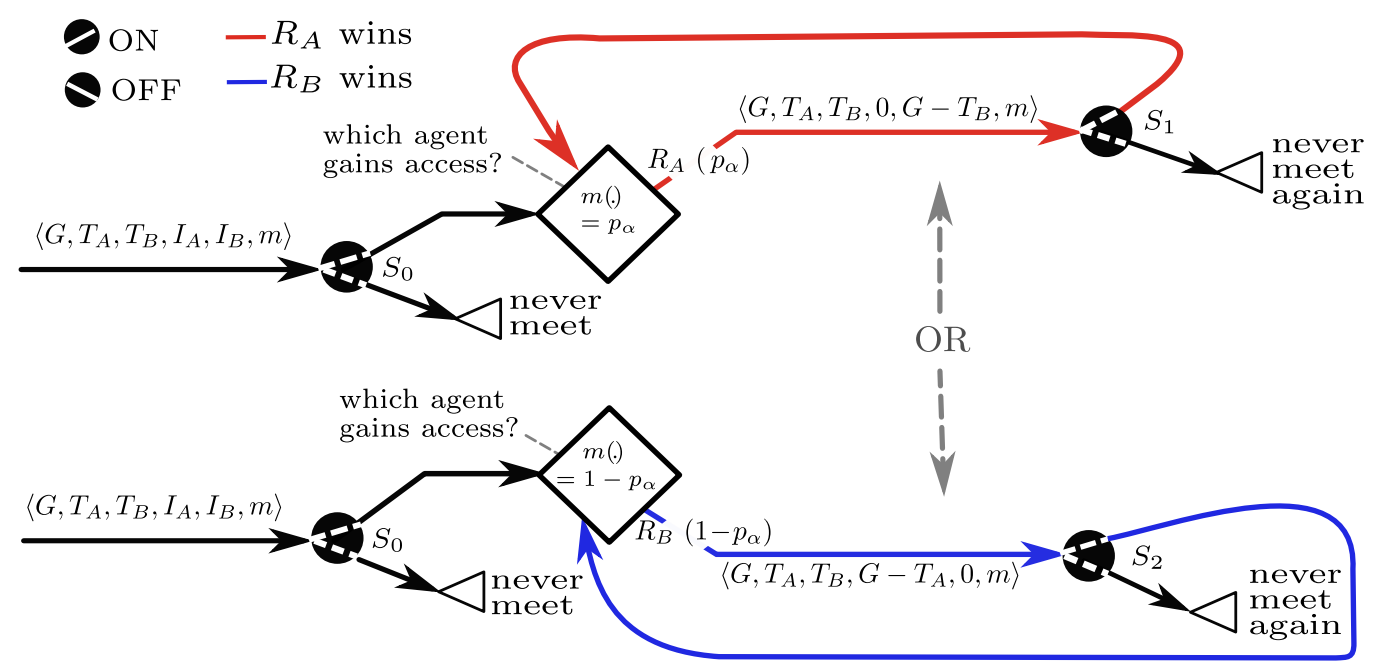

Fig. 29. Decision tree encoding when arbitration mechanism is static deterministic.

There can be only two possible decision tree encodings depending upon which agent is the pre-determined winner. It can be observed from Figure 29 that in each of these encodings there are only two possible termination paths:-

- $\mathrm{S} 0$ is $\mathrm{OFF}$, i.e. answers 'NO' encounter.

- $\mathrm{S} 0$ is $\mathrm{ON}$ but $\mathrm{S} 1$ is OFF (answers 'NO' encounter) when $R_{A}$ is the predetermined winner or $\mathrm{S} 2$ is $\mathrm{OFF}$ (answers 'NO' encounter) when $R_{B}$ is the predetermined winner.

Whenever the interaction sequence misses the above termination paths it will enter into an infinite sequence of future interferences. The non-termination paths will be followed when the following conditions are true:-

- $\mathrm{S} 0$ is $\mathrm{ON}$

- $\mathrm{S} 1$ is $\mathrm{ON}$ and $R_{A}$ is the pre-determined winner, or $\mathrm{S} 2$ is $\mathrm{ON}$ and $R_{B}$ is the pre-determined winner. 


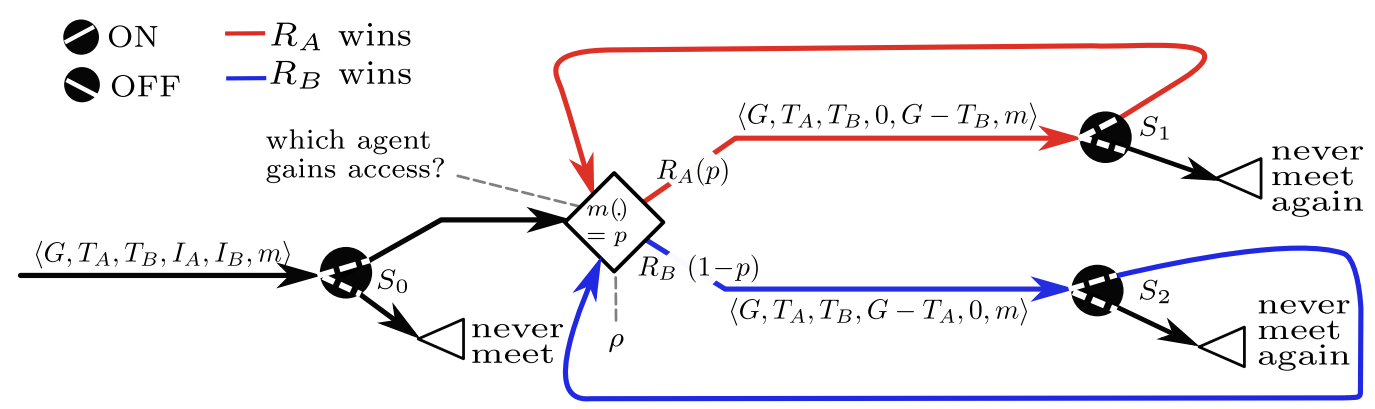

Fig. 30. Decision tree encoding when the arbitration mechanism is static probabilistic.

4. Static probabilistic - Figure 30 represents the decision tree encoding of a static probabilistic arbitration model. The only difference from the previous model is that here the winner is not pre-determined for the entire sequence of interaction, rather it is determined at every occurrence of an encounter. Since the arbitration is static, by definition no information from the current encounter is utilized in determining the winner and there is some constant probability for a robot to gain access to the girdle. This is denoted by $p$ in Figure 30.

One important point to note in all static models is that there is just one interference point, because every time an encounter takes place the resource is arbitrated the same way, independent of any encounter information. Thus $\alpha=\beta=\gamma=\rho$. However, there are three distinct interference switches because of the same reason as is outlined in the proof of Theorem 3 .

It can be observed from Table IV that in case of a static deterministic model the terminating strings can have a maximum length of one, while static probabilistic model can generate a terminating string of maximum length two. Also, the strings generated from static arbitration is a subset of those generated from dynamic deterministic arbitration and their corresponding mapping is shown in Table IV. 
Table III. Strings generated from a dynamic probabilistic model

\begin{tabular}{|c|c|c|c|}
\hline $\mathbf{S}_{0}$ & $\mathbf{S}_{1}$ & $\mathbf{S}_{2}$ & Strings \\
\hline$\diamond \mathrm{OFF}$ & - & - & $\lambda$ \\
\hline$\approx \mathrm{ON}$ & $\approx \mathrm{ON}$ & ८OFF & $\{\alpha, \alpha \beta, \alpha \beta \beta \cdots, \cdots\}=\alpha \beta^{*}$ \\
\hline$\approx \mathrm{ON}$ & ८OFF & $\approx \mathrm{ON}$ & $\{\alpha, \alpha \gamma, \alpha \gamma \gamma \cdots, \cdots\}=\alpha \gamma^{*}$ \\
\hline $\boldsymbol{\theta O N}$ & $\Theta \mathrm{ON}$ & $\Theta \mathrm{ON}$ & $\begin{array}{l}\alpha \beta \beta \beta \cdots, \alpha \beta \gamma \beta \beta \cdots, \alpha \beta \gamma \gamma \cdots, \\
\alpha \gamma \beta \gamma \cdots, \alpha \gamma \gamma \beta \cdots, \cdots=\alpha(\beta+\gamma)^{\infty}\end{array}$ \\
\hline
\end{tabular}

C. Interference Patterns Generated from Models of Interference

After obtaining strings of interference patterns the next question to ask is Can we predict the relative likelihood of occurrence of a string of interference pattern given a set of such patterns and the state of the interference switches? In other words, given the state of the interference switches and a set of strings representing interference patterns, can we evaluate the relative likelihood of occurrence of each of these strings? It was found that such questions can be answered.

A fact about the arbitration models discussed so far is that all of them are memoryless. This means that any future interference just depends on how the current interference is resolved and is independent of the way how past interferences occurred. Thus the probability of occurrence of any character in an interference pattern obeys the Markovian property. This property is fundamental in finding the probability of occurrence of any string of interference pattern. The following theorem results:-

Theorem 4. Given an arbitration model, the relative likelihood of occurrence of any string of interference pattern is equal to the product of the probabilities of occurrence of the characters in that string. 
Table IV. Strings generated from a static arbitration model

\begin{tabular}{|c|c|c|c|c|c|}
\hline $\begin{array}{l}\text { Static arbitra- } \\
\text { tion }\end{array}$ & $\mathbf{S}_{0}$ & $\mathbf{S}_{1}$ & $\mathbf{S}_{2}$ & Strings & $\begin{array}{l}\text { String mapping } \\
\text { from dynamic de- } \\
\text { terministic model }\end{array}$ \\
\hline $\begin{array}{l}\text { Deterministic / } \\
\text { probabilistic }\end{array}$ & SOFF & - & - & $\lambda$ & $\lambda$ \\
\hline $\begin{array}{l}\text { Deterministic / } \\
\text { probabilistic }\end{array}$ & $\approx \mathrm{ON}$ & sOFF & - & $\rho$ & $\alpha$ \\
\hline $\begin{array}{l}\text { Deterministic / } \\
\text { probabilistic }\end{array}$ & $\approx \mathrm{ON}$ & - & SOFF & $\rho$ & $\alpha$ \\
\hline Probabilistic & $\Theta O N$ & $\approx O N$ & SOFF & $\rho \rho$ & $\alpha \beta$ \\
\hline Probabilistic & $\vartheta \mathrm{ON}$ & SOFF & $\vartheta \mathrm{ON}$ & $\rho \rho$ & $\alpha \gamma$ \\
\hline $\begin{array}{l}\text { Deterministic / } \\
\text { probabilistic }\end{array}$ & $\Theta \mathrm{ON}$ & $\Theta \mathrm{ON}$ & - & $\rho^{\infty}$ & $\alpha \beta^{\infty}, \alpha \beta(\gamma)^{\infty}, \alpha(\beta \gamma)^{\infty}$ \\
\hline $\begin{array}{l}\text { Deterministic / } \\
\text { probabilistic }\end{array}$ & $\approx \mathrm{ON}$ & - & $\approx \mathrm{ON}$ & $\rho^{\infty}$ & $\alpha(\gamma)^{\infty}, \alpha \gamma(\beta)^{\infty}, \alpha(\gamma \beta)^{\infty}$ \\
\hline
\end{tabular}


Table V. String mapping between dynamic deterministic and dynamic probabilistic models

\begin{tabular}{|l|l|}
\hline $\begin{array}{l}\text { Strings from } \\
\text { dynamic prob- } \\
\text { abilistic }\end{array}$ & Strings from dynamic deterministic \\
\hline$\lambda$ & $\lambda$ \\
\hline$\alpha \beta^{*}$ & $\alpha, \alpha \beta, \alpha \beta^{\infty}$ \\
\hline$\alpha \gamma^{*}$ & $\alpha, \alpha \gamma, \alpha \gamma^{\infty}$ \\
\hline$\alpha(\beta+\gamma)^{\infty}$ & $\alpha \beta(\gamma)^{\infty}, \alpha(\beta \gamma)^{\infty}, \alpha \gamma(\beta)^{\infty}, \alpha(\gamma \beta)^{\infty}$ \\
\hline
\end{tabular}

Proof. Any single interference arbitrated at an interference point is represented by a character. Because occurrence of an encounter follows Markovian property, no information from past interferences is used to arbitrate the current encounter. Hence every interference is an independent event. Thus the overall probability of a series of independent interferences is equal to the product of their individual probabilities.

Using Theorem 4, the probability of occurrence of any string, say $\alpha \beta^{n}$ is given by $\operatorname{Pr}\left(\alpha \beta^{n}\right)=1 . p_{\alpha} \cdot\left(1-p_{\beta}\right)^{n-1}$, where $p_{\alpha}, p_{\beta}$ are the probabilities with which a robot will be selected as the winner for $\alpha$ and $\beta$ encounters respectively and $n$ is the length of the string. As long as the probability of a winner at an interference point is in the range $0<p<1$, the probability of occurrence of a string of interference pattern tends to 0 as the length ' $n$ ' of the string tends to infinity. A longer interference pattern is less likely to occur compared to a shorter one. However on comparing strings from different arbitration models a paradoxical result follows. In a deterministic model probability $p \in\{0,1\}$. Thus if $p=1$, then the probability of the interference pattern tends to infinity when $n \rightarrow \infty$, however when $p=0$, this probability tends to zero 
as $n \rightarrow \infty$. Thus the probability of occurrence of any string varies at the limit when $n \rightarrow \infty$ for the two arbitration models.

These differences are outlined below. Moreover the reason why we only take dynamic deterministic and dynamic probabilistic models into consideration is because strings generated from a static deterministic and static probabilistic models are subsets of those from a dynamic deterministic model. The strings mentioned below correspond to those in Table VI. The mapping of the strings from the dynamic probabilistic model to those generated by the dynamic deterministic model can be seen in Table $\mathrm{V}$.

1. String $\alpha \beta^{*}$

Dynamic Probabilistic

$$
\lim _{n \rightarrow \infty} \operatorname{Pr}\left(\alpha \beta^{n}\right)=\lim _{n \rightarrow \infty} 1 \cdot p_{\alpha} \cdot\left(1-p_{\beta}\right)^{n-1}=0, \forall p_{\alpha}, p_{\beta} \text { where } 0<\left\{p_{\alpha}, p_{\beta}\right\}<1
$$

Dynamic Deterministic

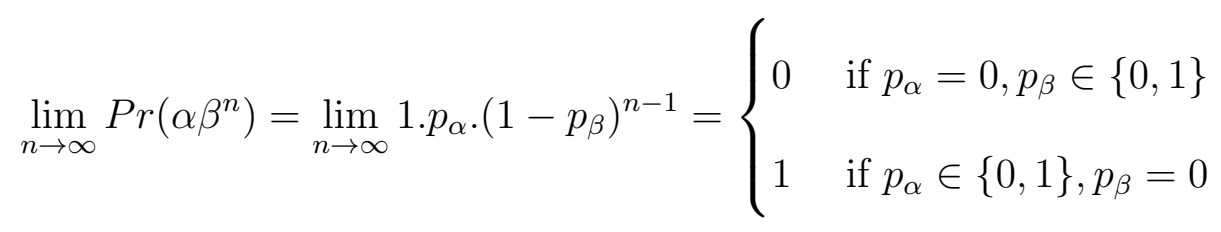

2. String $\alpha \gamma^{*}$

Dynamic probabilistic

$$
\begin{gathered}
\lim _{n \rightarrow \infty} \operatorname{Pr}\left(\alpha \gamma^{n}\right)=\lim _{n \rightarrow \infty} 1 .\left(1-p_{\alpha}\right) \cdot\left(1-p_{\gamma}\right)^{n-1}=0, \forall p_{\alpha}, p_{\gamma} \\
\text { where } 0<p_{\alpha}<1 \text { and } 0<p_{\gamma}<1
\end{gathered}
$$


Dynamic deterministic

$$
\lim _{n \rightarrow \infty} \operatorname{Pr}\left(\alpha \gamma^{n}\right)=\lim _{n \rightarrow \infty} 1 .\left(1-p_{\alpha}\right) .\left(1-p_{\gamma}\right)^{n-1}= \begin{cases}0 & \text { if } p_{\alpha}=1, p_{\beta} \in\{0,1\} \\ 0 & \text { if } p_{\alpha} \in\{0,1\}, p_{\gamma}=1 \\ 1 & \text { if } p_{\alpha}=0, p_{\gamma}=0\end{cases}
$$

3. String $\alpha(\beta+\gamma)^{\infty}$ - Comparing one of the mapped strings of the dynamic deterministic model with those of the dynamic probabilistic model, we find similar differences at the limits of $n \rightarrow \infty$. Considering one such mapped string, say $\alpha \beta(\gamma)^{\infty}$, we get:

Dynamic probabilistic -

$$
\begin{gathered}
\lim _{n \rightarrow \infty} \operatorname{Pr}\left(\alpha \beta \gamma^{n}\right)=\lim _{n \rightarrow \infty} 1 \cdot p_{\alpha} \cdot p_{\beta} \cdot\left(1-p_{\gamma}\right)^{n-2}=0, \forall p_{\alpha}, p_{\beta}, p_{\gamma} \\
\text { where } 0<p_{\alpha}<1,0<p_{\beta}<1 \text { and } 0<p_{\gamma}<1
\end{gathered}
$$

Dynamic deterministic -

$$
\lim _{n \rightarrow \infty} \operatorname{Pr}\left(\alpha \beta \gamma^{n}\right)=\lim _{n \rightarrow \infty} 1 \cdot p_{\alpha} \cdot p_{\beta} \cdot\left(1-p_{\gamma}\right)^{n-2}= \begin{cases}0 & \text { if } p_{\alpha}=0,\left(p_{\beta}, p_{\gamma}\right) \in\{0,1\} \\ 0 & \text { if } p_{\beta}=0,\left(p_{\alpha}, p_{\gamma}\right) \in\{0,1\} \\ 0 & \text { if } p_{\gamma}=1,\left(p_{\alpha}, p_{\gamma}\right) \in\{0,1\} \\ 1 & \text { if }\left(p_{\alpha}, p_{\beta}\right)=1, p_{\gamma}=0\end{cases}
$$

Thus for a probabilistic model the probability of occurrence of a string always tends to 0 when the length of the string tends to infinity. However in a deterministic model for certain probability combinations of individual characters (representing an interference), the probability of occurrence of the entire string tends to 1 even when string length tends to infinity. 
Another important result for strings belonging to the set $\alpha(\beta+\gamma)^{\infty}$ is the relative probability of occurrence of one string over another. To find this we will first give the following definition:-

Common suffix - Two strings of characters $x_{1}, x_{2}, \cdots, x_{i-1}, x_{i}, \cdots x_{m}$ and $y_{1}, y_{2}, \cdots, y_{i-1}, y_{i}, \cdots y_{n}$ are said to have a common suffix $Q$, if $x_{i-1}, x_{i}, \cdots x_{m} \equiv$ $y_{i-1}, y_{i}, \cdots y_{n}=Q$.

The probability of occurrence of a string with a common suffix $Q$ can be defined as follows:-

$$
\begin{aligned}
\operatorname{Pr}\left(s_{0}, s_{1}, \cdots, s_{i+1}\right) & =\operatorname{Pr}\left(s_{0}, \cdots, s_{i-1}\right) \cdot \operatorname{Pr}\left(s_{i}, s_{i+1, \cdots s_{m}}\right) \\
& =\operatorname{Pr}\left(s_{0}, \cdots, s_{i-1}\right) \cdot \operatorname{Pr}(Q)
\end{aligned}
$$

where $\operatorname{Pr}(Q)=\prod_{k=i-1}^{m} \operatorname{Pr}\left(s_{k}\right)$ and

$$
\operatorname{Pr}\left(s_{k}\right)= \begin{cases}1-p_{\beta} & \text { if } s_{k}=s_{k+1}=\beta, \\ p_{\beta} & \text { if } s_{k}=\beta \text { and } s_{k+1}=\gamma \\ 1-p_{\gamma} & \text { if } s_{k}=s_{k+1}=\gamma, \\ p_{\gamma} & \text { if } s_{k}=\gamma \text { and } s_{k+1}=\beta .\end{cases}
$$

Thus if we can identify such a common suffix $Q$ between any two strings, we can express the relative probability of occurrences of these strings as:

$$
\frac{\operatorname{Pr}\left(x_{0}, x_{1}, \cdots, x_{i+1}\right)}{\operatorname{Pr}\left(y_{0}, y_{1}, \cdots, y_{i+1}\right)}=\frac{\operatorname{Pr}\left(x_{0}, \cdots, x_{i-1}\right) \cdot \operatorname{Pr}\left(x_{i}, x_{i+1}\right)}{\operatorname{Pr}\left(y_{0}, \cdots, y_{i-1}\right) \cdot \operatorname{Pr}\left(y_{i}, y_{i+1}\right)}=\frac{\operatorname{Pr}\left(x_{0}, \cdots, x_{i-1}\right)}{\operatorname{Pr}\left(y_{0}, \cdots, y_{i-1}\right)} .
$$


A few interesting examples are shown below to illustrate the significance of this result.

(a) Given two strings $a=\alpha \beta \beta \beta \cdots m$ characters and $b=\alpha \gamma \beta \beta \cdots n$ characters, where $m \geq n$ find the relative probability of occurrence of the two strings. $\operatorname{Pr}(a)=1 \cdot p_{\alpha} \cdot\left(1-p_{\beta}\right)^{n-1}=1 \cdot p_{\alpha} \cdot\left(1-p_{\beta}\right)^{m-(n-2)} \cdot\left(1-p_{\beta}\right)^{n-2}$. $\operatorname{Pr}(b)=1 .\left(1-p_{\alpha}\right) \cdot p_{\gamma} \cdot\left(1-p_{\beta}\right)^{n-2}$.

Hence,

$$
\frac{\operatorname{Pr}(a)}{\operatorname{Pr}(b)}=\frac{p_{\alpha} \cdot\left(1-p_{\beta}\right)^{m-(n-2)}}{\left(1-p_{\alpha}\right) \cdot p_{\gamma}} .
$$

(b) Give strings $a=\alpha(\beta \beta)(\beta \beta) \cdots$ and $b=\alpha \gamma \gamma \cdots$, can their relative probability of occurrence be equal although they have different symbols? If $\operatorname{Pr}(\beta \beta)=\operatorname{Pr}(\gamma)$, then their probabilities of occurrence will be equal. This is equivalent to saying that for every two occurrence of the symbol (visiting interference point) $\beta$ there will be only one occurrence of symbol $\gamma$.

D. Relative Cost of an Interference Pattern

Cost of an interference can be defined as the time spent in arbitrating an encounter.

\section{Cost $\propto$ Arbitration time.}

Thus the cost of an interference pattern is the sum of the costs of all the individual interferences, each of which is represented by a character. Let cost of an individual interferences $i$ be denoted by the functions $C\left(x_{i}\right)$, where $x_{i}$ is the girdle percentage of encounter, then the cost for any interference pattern say, $\alpha \beta^{\infty}$ is:

$$
\operatorname{Cost}\left(\alpha \beta^{\infty}\right)=C\left(x_{\alpha}\right)+C\left(x_{\beta}\right)+\left[C\left(x_{\beta}\right)^{\infty}+\cdots\right]
$$


Table VI. Relative likelihood of occurrence of a string generated from a dynamic probabilistic model

\begin{tabular}{|l|l|}
\hline Strings & Probabilities \\
\hline$\lambda$ & - \\
\hline$\alpha \beta^{*}$ & $\operatorname{Pr}\left(\alpha \beta^{n}\right)=1 \cdot p_{\alpha} \cdot\left(1-p_{\beta}\right)^{n-1}$ \\
\hline$\alpha \gamma^{*}$ & $\operatorname{Pr}\left(\alpha \gamma^{n}\right)=1 .\left(1-p_{\alpha}\right) \cdot\left(1-p_{\gamma}\right)^{n-1}$ \\
\hline $\operatorname{Pr}\left(s_{0}, s_{1}, \cdots, s_{i+1}\right)$ & $=\operatorname{Pr}\left(s_{0}, \cdots, s_{i-1}\right) \cdot \operatorname{Pr}\left(s_{i}, s_{i+1}\right)$ \\
& $=\operatorname{Pr}\left(s_{0}, \cdots, s_{i-1}\right) \cdot Q$
\end{tabular} \mid \begin{tabular}{ll}
$1-p_{\beta} \quad$ if $s_{i}=s_{i+1}=\beta$ \\
$p_{\beta} \quad$ if $s_{i}=\beta$ and $s_{i+1}=\gamma$ \\
$1-p_{\gamma}$ & if $s_{i}=s_{i+1}=\gamma$ \\
$p_{\gamma}$ & if $s_{i}=\gamma$ and $s_{i+1}=\beta$ \\
\hline
\end{tabular}




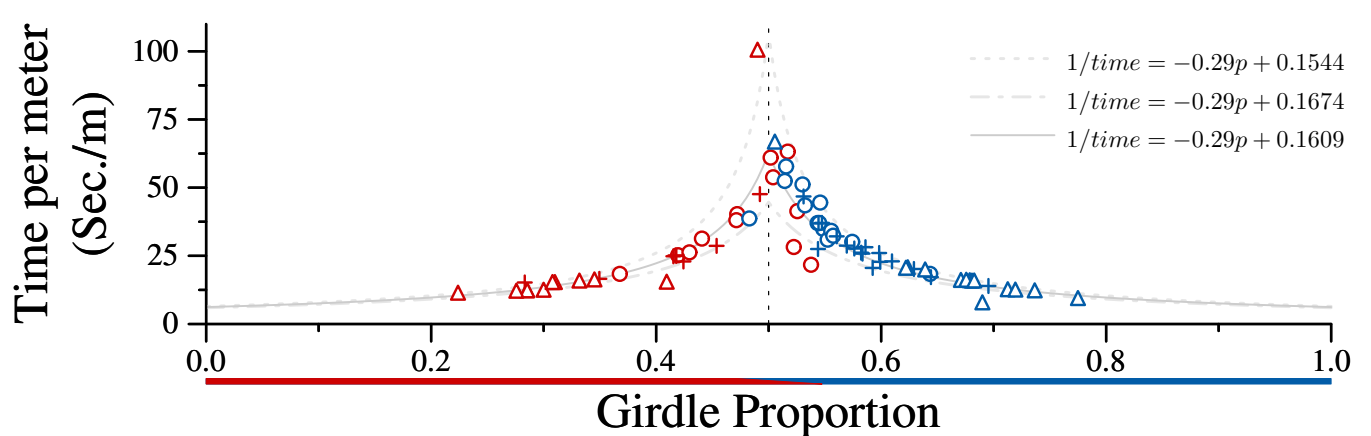

Fig. 31. Cost of an interference when arbitration mechanism is dynamic is proportional to the arbitration time and is at its maximum when the encounter is near the center of the girdle.

The cost function $C\left(x_{i}\right)$ for an interference $i$ is evaluated based on how the encounter is arbitrated. For example, a static arbitration takes a constant amount of time to select the winner, while the cost function of a dynamic arbitration might have some probability distribution on the position of encounter. In case of rational aggression it was found that the time taken for arbitration, hence the cost has a probability distribution as shown in Figure 31. The time of arbitration can be empirically fitted to the following line and this can be used for evaluating $C\left(x_{i}\right)$ :-

$$
\frac{1}{\text { time }}=-0.29 x_{i} \%+0.1609
$$

Details of this fit is mentioned in Chapter IV. 


\section{CHAPTER IX}

\section{BAYESIAN APPROACH TO RESOURCE ARBITRATION}

\section{A. Motivation for Bayesian Approach to Assessment}

The motivation for designing the various forms of binary robot arbitration mechanisms came from biological contests by animals. Several game theoretic models in literature are applicable to such contests. As per [8] game theory is the study of the ways in which strategic interactions among agents produce outcomes which maximizes the utility of each agent. In a game an agent assesses how an opponent will respond to its actions. Among all possible actions that an agent can take in response to every possible strategy used by the opponent, an agent chooses an action which is best suited for its own welfare. In none of the binary robot arbitration models seen so far there is any explicit assessment involved, nor do they make any choice of strategy based on assessment. Hence, clearly none of our binary robot arbitrations can be termed as a game. Thus none of the game theoretic models can be used to model such arbitration. However since all these models were inspired by models of animal contests we would still be interested in exploring the similarities between some of the characteristics of game theoretic models and our binary robot arbitration models.

Devi Stuart-Fox [9] provides a comparison between the main types of game theoretic models applicable to extended animal contests. She also outlines the factors which go about in deciding which model is most suitable for predicting the relationship between contestants' fighting ability and the duration, intensity and structure of the contest. The factors to consider are:-

1. Whether the behaviors of contestants match in terms of intensity, rate, energy expenditure or other factors. 
2. Whether the individual fighting ability or asymmetries in fighting ability determines the duration and outcome of the contest, where asymmetry is determined by assessing the opponent's ability to put up a fight.

3. Whether there is escalation within contest phases, where a phase is defined as certain periods in the contest characterized by similar intensity behavior.

We do see the existence of some of these characteristics in our current robot arbitration models - both 'rational aggression' and 'cutting your losses' mechanisms involve implicit escalation when competing for shared resource. In case of robots traversing a shared space the amount of local task investment can be considered proportional to its ability to compete for shared space. We saw that a difference in the amount of task investment which is analogous to asymmetry in fighting abilities determines the duration of the contest. Moreover, there is a negative correlation between these two factors. This characteristic was observed when the robots met at the center of the girdle and it took increasingly more time for one of them to get right of way. The lesser the difference in the local investment of the robots, more is the time taken to arbitrate the resource.

An aspect of game theoretic models which is not investigated in any of the robot arbitration models discussed so far is arbitration through assessment of opponent's fighting ability. One such example of a game theoretic model where an assessment is done to decide the outcome of an extended animal contest is the 'sequential assessment model'. SAM was first proposed by Enquist and Leimar [7], [10]. It obeys the characteristics proposed by Devi Stuart-Fox as follows:-

- The behavior of contestants are unmatched in rate, intensity or other factors.

- The decision to continue fighting is based on the assessment of opponents relative fighting ability. 
- There is no escalation between contest phases.

\section{B. Assessment of Local Investment in Binary Robot Arbitration}

The idea behind the SAM model is that every time an agent interacts with its opponent it obtains information about its opponent's fighting ability. The agent also has a prior knowledge of its opponent's fighting ability, which is equivalent to its belief of the opponent's strength, even before the contest has started. This belief gets better and closer to the true fighting ability of the opponent as more observations of the opponent's actions are made during the course of the fight. Because of the error associated with any observation an agent cannot accurately assess its opponent's ability at the very first interaction and the only way to enhance the accuracy is repeated observations. Thus the two main factors based on which an agent determines whether to continue the contest are: the estimated fighting ability of the opponent and the error in the observations which in turn determines the uncertainty associated with the current estimate. Based on the information acquired, the agent then makes a decision so as to maximize its expected utility.

Based on the characteristics of the SAM model, we wanted to explore the idea of how well can the minimalist robots assess their opponent's fighting ability during a binary interaction. In case of the present robot navigation task, fighting ability is defined as the amount of local investment a robot makes while crossing through the shared girdle space. If the robots $R_{A}$ and $R_{B}$ have traversed distances $x_{A}$ and $x_{B}$ respectively inside the girdle before encounter, then their respective local investments are $x_{A}$ and $x_{B}$. Thus the difference in their fighting abilities is $x_{A}-x_{B}$. The robots do not have a notion of its opponent's local investment. However in each successive interactions it can measure the distance gained or lost with respect to its first bump 
position in that particular interaction sequence. After the first bump at positions $x_{A}$ and $x_{B}$ by the two robots $R_{A}$ and $R_{B}$ respectively, they back a distance $\frac{1}{x_{A}}$ and $\frac{1}{x_{B}}$. Thereafter they move forward again until they bump into each other. The local investment $x_{A}$ and $x_{B}$ will almost never be equal even if they meet at the center of the girdle, this is because of the inherent noise in the sensors. Thus one of the robots will back more compared to the other. This difference is: $\frac{1}{x_{A}}-\frac{1}{x_{B}}$ and they will bump exactly at the mid point of this difference, assuming there is no noise. This measurement is termed as $\Delta$ and because of the way $\Delta$ is computed it encodes the asymmetries in the local investments of the robots. In an ideal noise free scenario, True $\Delta=\frac{1}{2} \cdot\left(\frac{1}{x_{A}}-\frac{1}{x_{B}}\right)$. From henceforth true $\Delta$ will be represented as $\Delta_{T}$ and any observed $\Delta$ as $\Delta_{O} . \Delta_{T}$ is the ground truth for comparison with $\Delta_{O}$.

The steps in obtaining $\Delta_{O}$ observations with each successive interactions can be outlined as follows:-

1. Each robot records their first bump positions $b_{1_{A}}$ or $b_{1_{B}}$ with respect to their own reference frames. Thereafter all measurements of loss or gain in distance during arbitration is done with respect to this first recorded bump position.

2. Each robot then backs a distance inversely proportional to their initial bump position. Next they move forward until they bump into each other. This new bump position is recorded as $b_{i_{A}}$ and $b_{i_{B}}$ respectively for both $R_{A}$ and $R_{B}$ for bump $i$. The gain or loss in the distance with respect to $b_{1}$ is also calculated. Thus $\Delta_{O}=b_{i_{A}}-b_{1_{A}}$ or $\Delta_{O}=b_{i_{B}}-b_{1_{B}}$. In an ideal noise free scenario, $\Delta_{O}$ $=\Delta_{T}$. A positive $\Delta_{O}$ denotes a gain in distance and a higher local investment than its opponent. The converse is true for a negative $\Delta_{O}$ observation.

3. When $\Delta_{O}$ measured is positive, the robot moves back $2 \Delta_{O}$ distance. The opponent then waits for a time equal to what it takes to cover $2 \Delta_{O}$ distance by the 
other robot. This step ensures that the effects of the $\Delta_{O}$ s are nullified before the next interaction, that is before the next observation of $\Delta_{O}$.

4. From their respective positions each robot now backs a distance inversely proportional to their initial bump positions.

5. Next they navigate forward till they meet each other again. This new bump position is recorded and the steps from 2 onwards are repeated.

Because of the noise in the communication channel the $\Delta_{O} \neq \Delta_{T}$. Thus each robot needs to take repeated measurements in order to reduce the error in its observation.

An important aspect to be noted is that in case of the SAM model the measure of asymmetries in the fighting ability is based on the information about the fighting abilities transmitted during the contest. However our minimalist robots do not have any direct communication of their local investments (their fighting abilities). They still can assess the asymmetries in their local investments by measuring $\Delta_{O}$ s. In that sense our model is more general compared to SAM. In case of SAM, the error in the observed asymmetries would follow a Gaussian distribution centered around the true asymmetry in the fighting ability with large enough observations. The standard deviation depends on the error in the observations. In our model, the error in the $\Delta_{O}$ measurements follow a Gaussian distribution centered around the true $\Delta_{T}$ and with a standard deviation depending on the amount of error in the observations. Figure 32 shows this probability distribution.

In addition to the $\Delta_{O}$ observations, the robots also have a prior knowledge of the possible values of $\Delta_{O}$. They start with a uniform probability distribution over all possible a priori values of $\Delta_{O}$. This initial probability distribution is updated as the interaction sequence proceeds and successive values of $\Delta_{O}$ are measured. Baye's rule 
is used as follows to update the probability distribution of the measured $\Delta_{O}$ :

$$
\operatorname{Prob}\left(\Delta_{T} \mid \Delta_{O}\right)=\frac{\operatorname{Prob}\left(\Delta_{O} \mid \Delta_{T}\right) \cdot \operatorname{Prob}\left(\Delta_{T}\right)}{\operatorname{Prob}\left(\Delta_{O}\right)}
$$

Each application of Baye's rule answers the question - what is the most likelihood $\Delta_{T}$ given an observation $\Delta_{0_{i}}$ for the $i^{\text {th }}$ interaction?

The local investment of the opponent is computed from the observations by using the same equation used for $\Delta$ calculations. Hence the observed local investment can be calculated from the observed $\Delta$ as follows:

$$
\begin{gathered}
R_{A} \text { 's estimate }=\frac{1}{\frac{1}{b_{1}}-2 \Delta_{O_{A}}} \\
R_{B} \text { 's estimate }=\frac{1}{\frac{1}{b_{1}}-2 \Delta_{O_{B}}}, \\
\text { where } \Delta_{O_{A}}=\Delta_{O} \text { of } R_{A} \text { and } \Delta_{O_{B}}=\Delta_{O} \text { of } R_{B} .
\end{gathered}
$$

However the error in the estimates of the local investments do not follow a Gaussian distribution. This is because $\Delta$ is not directly proportional to the difference in the local investments, rather it is equal to the difference in the inverse of the investments.

Figures 33, 34 show the changing probability distribution of the estimates with each subsequent $\Delta_{O}$ measurements. In both cases the probability distribution gets peaked with increasing number of observations, re-enforcing the robot's belief about its opponent's local task investment. There is a subtle difference in the figures 33 and 34. From figure 33 we see that the estimates get closer to the true local investment of its opponent and with a higher probability. However in Figure 34 we see a bias in the measurement and although the probability gets peaked with more observations, it is not very close to the true local investment of $R_{A}$.

Figures 35(a) and 35(a) show how the estimates get closer to the true mean (true 


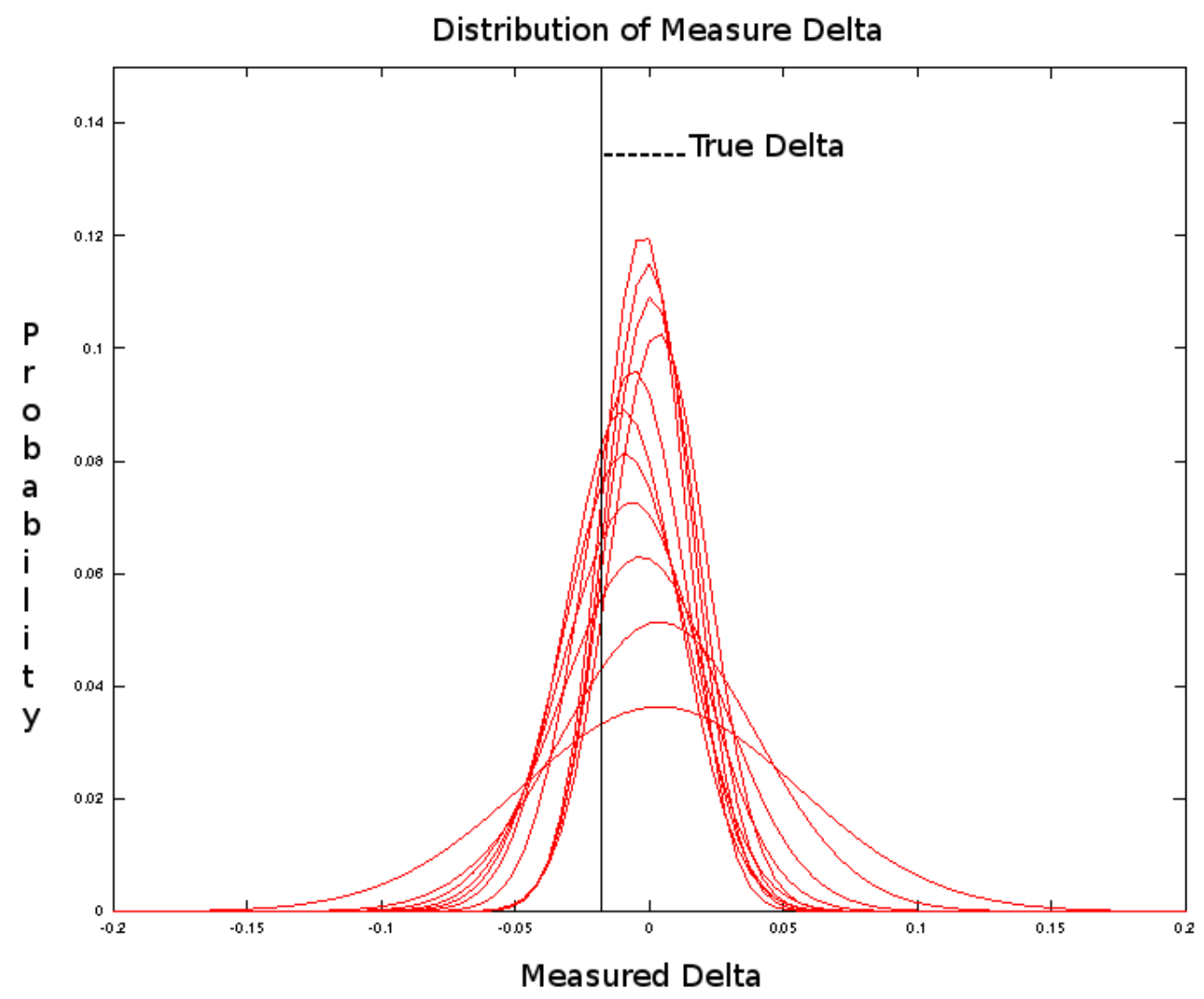

Fig. 32. Probability distribution of measured $\Delta$ for a given true $\Delta$. The distributions over a set of 11 observations are shown. The observed $\Delta$ gets closer to the true $\Delta$ with lesser standard deviation with each successive observation. 


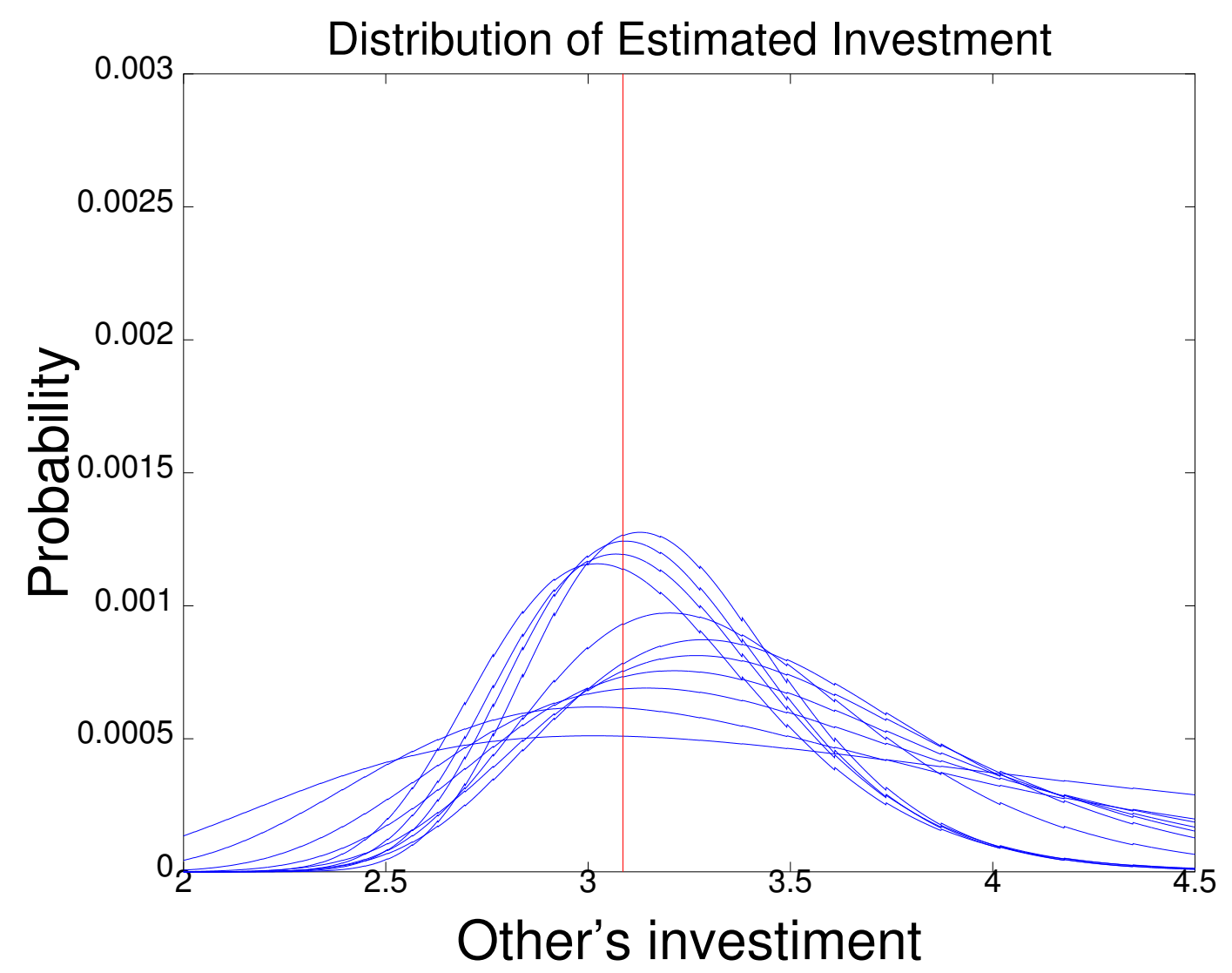

Fig. 33. Probability distribution of estimated local investment. Here $R_{A}$ estimates its opponent $R_{B}$ 's local investment. The distributions are over a set of 11 observations of $\Delta$ and the probability distribution of the estimate is updated with each successive observations. 


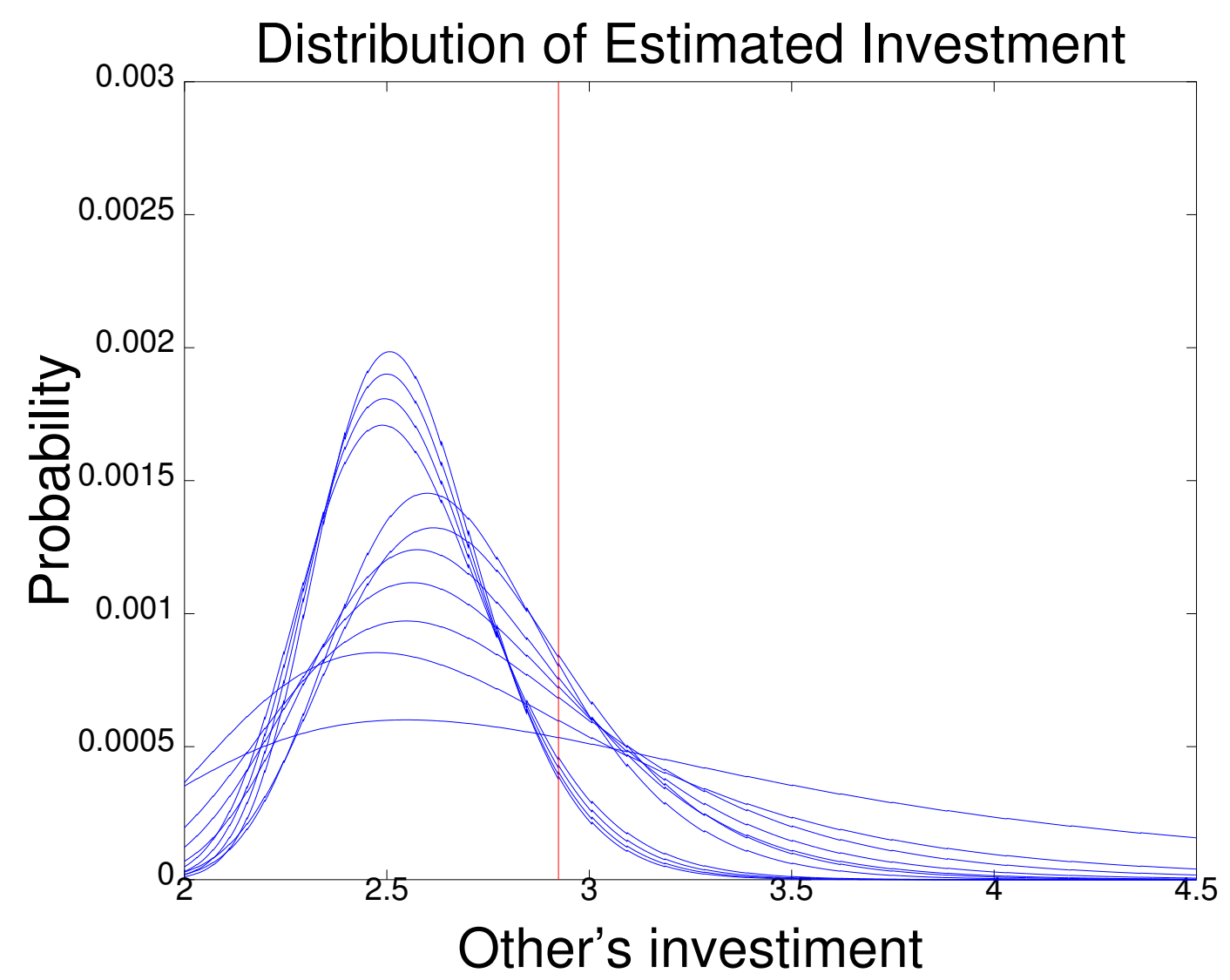

Fig. 34. Probability distribution of estimated local investment. Here $R_{B}$ estimates its opponent $R_{A}$ 's local investment. The distributions are over a set of 11 observations of $\Delta$ and the probability distribution of the estimate is updated with each successive observations. 


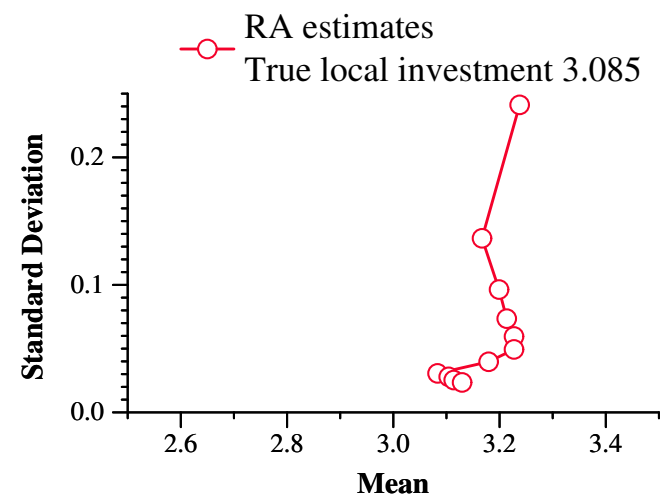

(a) $R_{A}$ estimates $R_{B}$

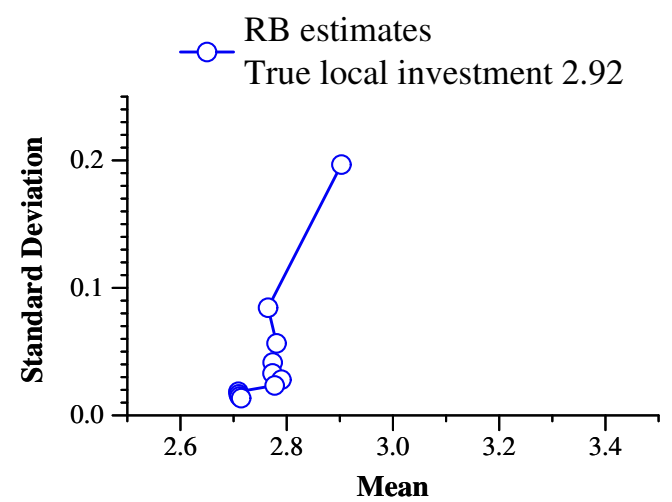

(b) $R_{B}$ estimates $R_{A}$

Fig. 35. Error in the estimation decreases with increased observation of $\Delta_{O}$.

local investment) with successive observations. The error in the estimates decreases by a factor of $\frac{\sigma}{\sqrt{n}}$, where $n$ is the number of $\Delta_{O}$ observations and $\sigma$ is the standard deviation from the mean. Since the decrease in the error is related by a factor of $\sqrt{n}$ to the number of observations, the return in terms of the decrease in the error from repeated observations decreases as the arbitration progresses. On the other hand the cost increases as the arbitration proceeds. Thus the factors which determines the decision to continue arbitration are the estimate of the difference in the local investment, the accuracy of the estimate and the cost associated with improving the accuracy. Figure 35(a) and 35(a) shows how the first two factors change as the arbitration proceeds. If we also consider the cost of each interaction then a decision could be made whether to switch to a retreat behavior given the current estimate and the associated error in its observation, or whether to continue arbitration trying to get more accurate estimate at the cost of increasing time of arbitration. A detailed analysis of this decision making process so as to maximize its utility is beyond the scope of the current research. Our main aim has been to introduce the concept of assessment in such minimalist binary robot arbitration process. 


\section{CHAPTER X}

\section{DISCUSSION AND FUTURE WORK}

\section{A. Overview}

In this work we saw a whole gamut of arbitration mechanisms starting from the most implicit one where arbitration is entirely without representation, to the deliberative coordinated ones, where the robots assess the asymmetries in their local task investments. In Chapter III we saw the categorization of arbitration mechanisms and a proposed taxonomy of binary robot arbitration mechanisms, such that any arbitration model can be represented as a quadruplet. We also saw the importance of outcome accuracy in rational arbitration and how different arbitration mechanisms can be scored on the accuracy scale. Chapter IV details the experimental framework for carrying out the study of arbitration models on physically grounded robots. We also extended this work to a simulation environment so as to analyze the effects of environment on spatial interference as we tune the environmental parameters. In Chapter V we did a comparative study of aggressive interaction and linear dominance. The results showed that the environment plays a key role in determining the ideal arbitration mechanism to follow so as to reduce arbitration time. In Chapter VI the comparative study was done on the simulation environment tweaking the task ratio and the girdle length parameters. The results from these experiments showed that a slight change in the task dynamics can drastically change the timings needed for task completion, which in turn is effected by the number of encounters and the arbitration time during each encounter. Chapter VII is one of the most conceptually challenging work where we mention about how an entire interference pattern can be encoded as a string with just three distinct symbols representing unique interference points. Finally 
in Chapter IX we came up with a deliberatively coordinated arbitration mechanism inspired by animal contests.

\section{B. Bayesian Inference Model}

Although we were able to show how our minimalist robots can assess the local investment of its opponent by the use of the Bayesian filter, there are several aspects yet to be considered in this model. We do not know when or how exactly the robots should decide to switch from continuing to arbitration behavior to the retreat behavior. What factors needs to be considered for maximizing the utility of an agent? To answer these questions further research is needed. Moreover, we saw that there was a bias in the estimations done by one of the robots (refer Figure 34). We conjecture that the bias might be an environmental property or might be due to an offset in the sensor readings of that particular robot. More runs of the assessment model might be able to answer this question. But at least we were successful in proposing an assessment based model inspired by a biological game theoretic model and reformulated from a Bayesian perspective.

\section{Cooperation versus Coordination}

A question which is worth asking is whether these arbitration mechanisms are cooperative or competitive? By definition 'cooperation' is the act of working together so as to achieve a common goal. In case of robots this would be to act together so as to minimize time or maximize the total number of tasks performed in a given time. 'Competition' on the other hand denotes selfishly working towards a goal which can-

not be shared. In case of the robot navigation task, this arises when they try crossing the shared girdle space. In the arbitration models where the robots make a decision 
based on their local investment, we saw reduced backing when investment is higher. Although there is no communication between the robots during the arbitration process, this behavior to stand ground is conceptually equivalent to implicit escalation for gaining ground, a flavor of selfish behavior for individual welfare. However, since the outcome of the arbitration models is a rational winner, on a global level the robots work towards the common goal of improving overall task performance. Thus at a local level they are competing for the shared resource, but on a global level they are cooperating to maximize their task performance. 


\section{CHAPTER XI}

\section{CONCLUSION}

Spatial interference is a commongly observed phenomena in robot navigation task. There has been several studies in the literature suggesting arbitration models. This research apart from suggesting arbitration models, identifies the key factors which determines the selection of an arbitration model. Our results demonstrate that several factors contribute to conflict resolution and its effectiveness:-

Cost vs. precision of the arbitration mechanism-Time and energy cost are incurred in resolving resource conflicts. This influences the utility of aggressive displays in the first place.

Properties of the shared resource for which the agents are competing - this affects, among other things, the cost of communicating its aggression and what constitutes a worthwhile investment.

The task which each agent is assigned to perform - this can be coupled through the shared resource. This coupling, effects individual and collective dynamics.

The inherent noise in the "communication" channel - noise plays a role in dynamic arbitration mechanisms: it can be beneficial in breaking symmetry, a situation which occurs when agents have identical aggression.

From all these facts we can conclude that there cannot be just one single best arbitration mechanism catering to all situations. We have also shown instances where a small variation of task ratio may cause a significant change in the task dynamics. With a prior knowledge of this entire task performance space, a single unfavorable interaction can be predicted beforehand, and by adding a wait to its task navigation, 
the robot can shift its performance to a more favorable region.

One of the main contributions of this work is representing patterns of interference as sets of strings with symbols drawn from a set of only three distinct symbols representing three distinct interference points. We provide decision tree encodings of the arbitration models and show how the choice of a particular path in the decision tree determines the string of interference pattern generated. Moreover, given a string of interference pattern we can also say the probability of its occurence and also its relative probabiltiy with respect to other strings of interference patterns. Morever cost of the interference pattern can be easily computed from these strings. Finally we also present a Bayesian approach for assessing an opponent robot's local task investment. Our method is inspired by the biological signaling behavior witnessed in extended animal contests. However our method is more general compared to the biological model which inspired its design.

\section{A. Contributions}

A complete understanding of a simple binary robot interference scenario will be beneficial to robotics research since spatial interference is ubiquitous in mobile robotics. Moreover a complete understanding of the dynamics of spatial interference in a simple domain can be extended to physical interference even in complex domains. When more than two robots are involved in spatial interference, interference can be considered to take place between two teams of robots, each team having one or more robots. Thus a multi-robot interference problem simplifies to a binary team of robot interference scenario which can be treated as a binary robot interference problem. The main contributions of this research are:-

1. A taxonomy of arbitration mechanisms for two-agent spatial interference, in- 
cluding a characterization of conflict resolution models is developed.

2. The notion of outcome accuracy and explicit consideration of interaction cost is introduced.

3. Previous claims in the literature have been refuted by showing that aggressive mode of arbitration is not always better than dominance and that environment plays a key role in interference and in determining the choice of the best mode of arbitration by agents.

4. It has been shown that memory of past interactions with respect to the task structure and properties of the resource can result in improved future task performance.

5. The research results also show that varying the properties of assigned task, the frequency of spatial interference and the cost incurred in its resolution varies significantly.

6. A new "minimalist" resource arbitration method is introduced which produces dynamic outcomes - albeit with comparatively high costs - suitable for simpler robots (with fewer sensors) than heretofore known.

7. An assessment model has been proposed whereby the robots by assesses its opponent's local investment by repeated observation in the gain or loss of distance in the shared space and by updating the probability of its estimate by applying Baye's rule.

8. Characterization of system performance have been provided where patterns of interference can be exactly expressed as sets of strings drawn from a set of just three symbols. The probability of occurrence of a string as well as its relative probability with respect to another string can be computed. Moreover, given a string of interference pattern one can predict the cost associated with that sequence of interaction. This analysis of the domain has the important (and 
rare) property of completeness, i.e., all possible abstract variations of the task are understood. 


\section{REFERENCES}

[1] R. T. Vaughan, K. Støy, G. S. Sukhatme, and M.J. Matarić, "Go ahead, make my day: Robot conflict resolution by aggressive competition," in From Animals to Animats: Proc. Simulation of Adaptive Behavior, Jean-Arcady Meyer, Alain Berthoz, Dario Floreano, Herbert L. Roitblat, and Stewart W. Wilson, Eds., Paris, France, Aug. 2000, pp. 491-500.

[2] S. Brown, M. Zuluaga, Y. Zhang, and R. T. Vaughan, "Rational aggressive behaviour reduced interferrence in a mobile robot team," in Proc. International Conference on Advanced Robotics, Seattle, WA, July 2005.

[3] M. Zuluaga and R. T. Vaughan, "Reducing spatial interference in robot teams by local-investment aggression," in Proc IEEE International Conference on Intelligent Robots and Systems, Edmonton, Alberta, August 2005.

[4] M. Enquist, "Communication during aggressive interactions with particular reference to variation in choice of behaviour," Animal Behaviour, vol. 33, no. 4, pp. $1152-1161,1985$.

[5] D. Goldberg and M. J. Matarić, "Interference as a tool for designing and evaluating multi-robot controllers," in Proc. AAAI National Conference on Artificial Intelligence, Providence, RI, July 1997, pp. 637-642.

[6] K. Lorenz, "On aggression.," in From Animals to Animats: Proc. Simulation of Adaptive Behavior, Harcourt, Brace, and World Inc., Eds., New York, 1966.

[7] M. Enquist and O. Leimar, "Evolution of fighting behaviour: Decision rules and assessment of relative strength," Journal of Theoretical Biology, vol. 102, no. 3, pp. $387-410,1983$. 
[8] D. Ross, "Game theory," in The Stanford Encyclopedia of Philosophy, Edward N. Zalta, Ed. Metaphysics Research Lab, CSLI, Stanford University, Stanford, CA, 2010.

[9] D. Stuart-Fox, "Testing game theory models: fighting ability and decision rules in chameleon contests," Proc. Royal Society B: Biological Sciences, vol. 273, no. 1593, pp. 1555-1561, 2006.

[10] M. Enquist, O. Leimar, T. Ljungberg, Y. Mallner, and N. Segerdahl, "A test of the sequential assessment game: fighting in the cichlid fish nannacara anomala," Animal Behaviour, vol. 40, no. 1, pp. 1 - 14, 1990. 


\section{VITA}

Name

Address
Tanushree Mitra

Department of Computer Science,

301 Harvey R. Bright Building,

College Station, TX 77843-3112

Email Address tmitra@cse.tamu.edu

\section{Education}

- Master of Science, Computer Science, Aug 2011, Texas A\&M University, College Station, TX

- Bachelor of Technology, Computer Science and Engineering, Sep 2005,Sikkim Manipal Institute of Technology, Sikkim, India 